DESY/02-214

hep-th/0303162

\title{
Massive Feynman diagrams and inverse binomial sums
}

\author{
A. I. Davydychev ${ }^{a, *}$ and M. Yu. Kalmykov ${ }^{b, c \dagger}$ \\ ${ }^{a}$ Institute for Nuclear Physics, Moscow State University, \\ 119992, Moscow, Russia \\ ${ }^{b}$ DESY-Zeuthen, Theory Group, Platanenallee 6, \\ D-15738 Zeuthen, Germany \\ ${ }^{c}$ BLTP, JINR, 141980 Dubna, Russia.
}

\begin{abstract}
When calculating higher terms of the $\varepsilon$-expansion of massive Feynman diagrams, one needs to evaluate particular cases of multiple inverse binomial sums. These sums are related to the derivatives of certain hypergeometric functions with respect to their parameters. We explore this connection and analytically calculate a number of such infinite sums, for an arbitrary value of the argument which corresponds to an arbitrary value of the off-shell external momentum. In such a way, we find a number of new results for physically important two-loop two- and three-point Feynman diagrams. The results are presented in terms of generalized polylogarithmic functions.
\end{abstract}

\footnotetext{
${ }^{*}$ Current address: Schlumberger, SPC, 110 Schlumberger Dr., MD-5, TX 77478, USA. Email address: davyd@thep.physik.uni-mainz.de

${ }^{\dagger}$ Supported by DFG under Contract SFB/TR 9-03 and in part by RFBR grant \# 04-02-17192. Email address: kalmykov@thsun1.jinr.ru
} 


\section{Introduction}

In many cases, the results of analytical calculation of Feynman diagrams can be represented as combinations of hypergeometric functions. However, the problem of constructing the $\varepsilon$-expansion of hypergeometric functions within dimensional regularization [1] (where $n=4-2 \varepsilon$ is the space-time dimension) is not completely solved. Rather often, results for the terms of the $\varepsilon$-expansion can be expressed in terms of polylogarithms [2] as well as Nielsen polylogarithms [3]. Recently it was demonstrated that new types of functions, harmonic polylogarithms [4] and multiple polylogarithms [5], appear in multiloop calculations. Furthermore, the nested sums [6] were proposed as a generalization of multiple polylogarithms, and the $\varepsilon$-expansion for a large class of hypergeometric function was constructed [7].

In this paper we study the multiple inverse binomial sums defined as ${ }^{1}$

$\Sigma_{a_{1}, \ldots, a_{p} ; b_{1}, \ldots, b_{q} ; c}^{i_{1}, \ldots, i_{p} ; j_{1}, \ldots, j_{q}}(u) \equiv \sum_{j=1}^{\infty} \frac{1}{\left(\begin{array}{c}2 j \\ j\end{array}\right)} \frac{u^{j}}{j^{c}}\left[S_{a_{1}}(j-1)\right]^{i_{1}} \ldots\left[S_{a_{p}}(j-1)\right]^{i_{p}}\left[S_{b_{1}}(2 j-1)\right]^{j_{1}} \ldots\left[S_{b_{q}}(2 j-1)\right]^{j_{q}}$,

where $S_{a}(j) \equiv \sum_{k=1}^{j} k^{-a}$ is the harmonic sum ${ }^{2}$ and $u$ is an arbitrary argument. In what follows, we will also use $z \equiv \frac{1}{4} u$ as the argument of the occurring hypergeometric functions. For sums of the type (1.1), the weight $\mathbf{J}$ can be defined as $\mathbf{J}=c+\sum_{k=1}^{p} a_{k} i_{k}+\sum_{k=1}^{q} b_{k} j_{k}$, whereas the depth can be associated with the sum $\sum_{k=1}^{p} i_{k}+\sum_{k=1}^{q} j_{k}$.

All multiple binomial sums (1.1) can be presented in terms of function $\psi(z)=\frac{\mathrm{d}}{\mathrm{d} z} \ln \Gamma(z)$ and its derivatives by means of the following relation

$$
\psi^{(k-1)}(j)=(-1)^{k}(k-1) !\left[\zeta_{k}-S_{k}(j-1)\right], \quad k>1
$$

where $\psi^{(k)}(z)$ is the $k$-th derivative of the $\psi$-function. In particular, for $k=1$ we have $\psi(j)=S_{1}(j-1)-\gamma_{E}$, where $\gamma_{E}$ is Euler's constant.

The sums (1.1) appear in the calculation of massive Feynman diagrams within several different approaches: for instance, as solutions of differential equations for Feynman amplitudes [9], through a nä̈ve $\varepsilon$-expansion of hypergeometric functions within Mellin-Barnes technique [10], or in the framework of recently proposed algebraic approach [11]. Physical applications include the one-, two- and three-loop massive Feynman diagrams with two massive cuts [12-17]. The case $u=1$ corresponds to the single-scale propagator-type diagrams [18]. Although there are many publications concerning harmonic series [19], only a limited number of results are available for the inverse binomial sums. Some particular results for $u=1,2,3$ can be extracted from [16,20]. The sums with $u=1,3$ are expressible in terms of an "odd" basis [21], whereas the case $u=2$ corresponds to an "even" basis [16, 22] $]^{3}$.

\footnotetext{
${ }^{1}$ Some particular results for the multiple binomial sums are presented in [8]. Those sums are defined similarly to Eq. (1.1), but with $\left(\begin{array}{c}2 j \\ j\end{array}\right)$ in the numerator, rather than in the denominator.

${ }^{2}$ Through the rest of this paper, the notations $S_{a}$ and $\bar{S}_{a}$ will always mean $S_{a}(j-1)$ and $S_{a}(2 j-1)$, respectively, even we do not mention this explicitly. When there are no sums of the type $S_{a}$ or $\bar{S}_{b}$ on the r.h.s. of Eq. (1.1), we shall put a "-" sign instead of the indices $(a, i)$ or $(b, j)$ of $\Sigma$, respectively.

"The connection between "sixth root of unity" [23] and "odd" / "even" bases was discussed in [16,24].
} 
Several new results for a generalization of the sums (1.1) are presented in [25]. However, only for special type of sums the analytical results are available [20,26,27],

$$
\sum_{j=1}^{\infty} \frac{1}{\left(\begin{array}{c}
2 j \\
j
\end{array}\right)} \frac{u^{j}}{j^{c}}=-\sum_{i=0}^{c-2} \frac{(-2)^{i}}{i !(c-2-i) !}(\ln u)^{c-2-i} \operatorname{Ls}_{i+2}^{(1)}(\theta)
$$

where $c \geq 2$,

$$
\begin{gathered}
\theta \equiv 2 \arcsin \left(\frac{1}{2} \sqrt{u}\right)=2 \arcsin \sqrt{z} \\
\operatorname{Ls}_{j}^{(k)}(\theta)=-\int_{0}^{\theta} \mathrm{d} \phi \phi^{k} \ln ^{j-k-1}\left|2 \sin \frac{\phi}{2}\right|, \quad \operatorname{Ls}_{j}(\theta)=\operatorname{Ls}_{j}^{(0)}(\theta)
\end{gathered}
$$

is the generalized log-sine function [2]. Some examples when such functions occur in the $\varepsilon$-expansion of Feynman diagrams can be found in Refs. [21,28-30]. Usually, the occurring angles (1.3), possess certain geometrical meaning [13].

The main aim of the present publication is the analytical calculation of inverse binomial sums (1.1), including some relevant examples of physically important Feynman diagrams.

The paper is organized as follows. In Section 2, employing the connection with the $\varepsilon$ expansion of hypergeometric functions, we obtain analytical results for sums of the type (1.1), valid for $u \leq 4(z \leq 1)$. Mainly the sums of the weights 3 and 4 are considered. In Section 3 the analytical continuation to other values of $u$ is constructed. Section 4 contains some applications of our results related to the $\varepsilon$-expansion of Feynman diagrams, mainly two-loop master integrals. In Appendix A we briefly summarize the relevant properties of the harmonic polylogarithms of complex arguments and related functions. In Appendix B we show how certain identities between hypergeometric functions can be used to establish relations between the corresponding sums. In Appendix $\mathrm{C}$ we have collected explicit results for inverse binomial sums of lower weights. In Appendix D relations between binomial, harmonic and inverse binomial sums are explored, and analytical results for multiple binomial sums up to weight 3 are presented. Appendix E contains a realistic example of a physical application of the considered integrals. (Remember to change when appendices are ready!)

\section{Inverse binomial sums and hypergeometric functions}

\subsection{The $\varepsilon$-expansion of the ${ }_{2} F_{1}$ function}

Here, our analysis is based on comparing two representations of hypergeometric function whose parameters depend on $\varepsilon$. One of them is the series representation in terms of the harmonic sums, whereas the second one is the exact result in terms of the functions related to the polylogarithms.

In particular, we consider the ${ }_{2} F_{1}$ hypergeometric function of a special type,

$$
{ }_{2} F_{1}\left(\begin{array}{c|c}
1+a_{1} \varepsilon, 1+a_{2} \varepsilon \\
\frac{3}{2}+b \varepsilon
\end{array} \mid z\right)=\sum_{j=0}^{\infty} \frac{u^{j}}{j !} \frac{\left(1+a_{1} \varepsilon\right)_{j}\left(1+a_{2} \varepsilon\right)_{j}(1+b \varepsilon)_{j}}{(2+2 b \varepsilon)_{2 j}}
$$


where $u=4 z,(\alpha)_{j} \equiv \Gamma(\alpha+j) / \Gamma(\alpha)$ is the Pochhammer symbol, and we have used the duplication formula $(2 \beta)_{2 j}=4^{j}(\beta)_{j}\left(\beta+\frac{1}{2}\right)_{j}$. To perform the $\varepsilon$-expansion we use the wellknown representation

$$
(1+a \varepsilon)_{j}=j ! \exp \left[-\sum_{k=1}^{\infty} \frac{(-a \varepsilon)^{k}}{k} S_{k}(j)\right]
$$

which yields

$$
\begin{aligned}
& { }_{2} F_{1}\left(\begin{array}{c|c}
1+a_{1} \varepsilon, 1+a_{2} \varepsilon \\
\frac{3}{2}+b \varepsilon
\end{array} \mid z\right)=\frac{2(1+2 b \varepsilon)}{u} \sum_{j=1}^{\infty} \frac{1}{\left(\begin{array}{c}
2 j \\
j
\end{array}\right)} \frac{u^{j}}{j}\left\{1+\varepsilon\left[\left(A_{1}+b\right) S_{1}-2 b \bar{S}_{1}\right]\right. \\
& +\varepsilon^{2}\left[2 b^{2}\left(\bar{S}_{2}+\bar{S}_{1}^{2}\right)-2 b\left(A_{1}+b\right) S_{1} \bar{S}_{1}-\frac{1}{2}\left(A_{2}+b^{2}\right) S_{2}+\frac{1}{2}\left(A_{1}+b\right)^{2} S_{1}^{2}\right] \\
& +\varepsilon^{3}\left[\frac{1}{6}\left(A_{1}+b\right)^{3} S_{1}^{3}-b\left(A_{1}+b\right)^{2} S_{1}^{2} \bar{S}_{1}-\frac{1}{2}\left(A_{1}+b\right)\left(A_{2}+b^{2}\right) S_{1} S_{2}\right. \\
& +2 b^{2}\left(A_{1}+b\right) S_{1}\left(\bar{S}_{2}+\bar{S}_{1}^{2}\right)+b\left(A_{2}+b^{2}\right) S_{2} \bar{S}_{1}+\frac{1}{6}\left(2 b^{3}-A_{1}^{3}+3 A_{1} A_{2}\right) S_{3} \\
& \left.\left.-\frac{4}{3} b^{3}\left(2 \bar{S}_{3}+3 \bar{S}_{2} \bar{S}_{1}+\bar{S}_{1}^{3}\right)\right]+\mathcal{O}\left(\varepsilon^{4}\right)\right\},
\end{aligned}
$$

where $A_{k} \equiv a_{1}^{k}+a_{2}^{k}$. Note that the coefficient of the $\varepsilon^{3}$ term can be represented as

$$
\begin{aligned}
& \frac{1}{6}\left(A_{1}^{3}+b^{3}\right) \mathcal{C}_{0}+2 b^{2}\left(A_{1}+b\right) \mathcal{C}_{1}+4 b^{3} \mathcal{C}_{2}+\frac{1}{2} b\left(A_{1}-b\right)\left(A_{1}-2 b\right) \mathcal{C}_{3} \\
& \quad+\frac{1}{2}\left(A_{1}^{2}-A_{2}\right)\left(A_{1}+b\right)\left(S_{1} S_{2}-S_{3}\right)+\frac{1}{2} b\left(A_{1}^{2}-A_{2}\right)\left(S_{3}-2 S_{2} \bar{S}_{1}\right),
\end{aligned}
$$

where we have introduced the following combinations of harmonic sums:

$$
\begin{aligned}
& \mathcal{C}_{0}=S_{1}^{3}-3 S_{1} S_{2}+2 S_{3}, \\
& \mathcal{C}_{1}=S_{1}^{3}-S_{1} S_{2}+S_{1}\left(\bar{S}_{1}^{2}+\bar{S}_{2}\right)-\frac{5}{2} S_{1}^{2} \bar{S}_{1}+\frac{3}{2} S_{2} \bar{S}_{1}, \\
& \mathcal{C}_{2}=\frac{3}{4} S_{1} S_{2}-\frac{3}{4} S_{1}^{3}+\frac{3}{2} S_{1}^{2} \bar{S}_{1}-S_{2} \bar{S}_{1}-\frac{1}{3}\left(2 \bar{S}_{3}+3 \bar{S}_{1} \bar{S}_{2}+\bar{S}_{1}^{3}\right), \\
& \mathcal{C}_{3}=S_{1}^{3}-2 S_{1}^{2} \bar{S}_{1}-S_{1} S_{2}+2 S_{2} \bar{S}_{1} .
\end{aligned}
$$

To completely define the $\varepsilon^{3}$ order of the expansion of ${ }_{2} F_{1}$, we need results for six combinations of sums, according to the number of independent combinations of the parameters $A_{i}$ and $b$.

Let us first consider the case $0 \leq u \leq 4(0 \leq z \leq 1)$. In this region the following parametrization can be used: $u=4 \sin ^{2} \frac{\theta}{2}\left(z=\sin ^{2} \frac{\theta}{2}\right)$, where $0 \leq \theta \leq \pi$. In the rest of this paper, we will use the short-hand notation

$$
L_{\theta} \equiv \ln \left(2 \cos \frac{\theta}{2}\right), \quad l_{\theta} \equiv \ln \left(2 \sin \frac{\theta}{2}\right) .
$$

For a few special cases, the $\varepsilon$-expansion of the ${ }_{2} F_{1}$ functions is known. First of all, the following relation holds [31]:

$$
{ }_{2} F_{1}\left(\begin{array}{c|c}
1+\varepsilon, 1-\varepsilon & \sin ^{2} \frac{\theta}{2} \\
\frac{3}{2} & \sin (\varepsilon \theta)
\end{array} .\right.
$$


Its expansion contains only the even powers of $\varepsilon$. Using it, it is easy to get

$$
\begin{aligned}
\sum_{j=1}^{\infty} \frac{1}{\left(\begin{array}{c}
2 j \\
j
\end{array}\right)} \frac{u^{j}}{j} S_{2} & =\frac{1}{6} \theta^{3} \tan \frac{\theta}{2}, \\
\sum_{j=1}^{\infty} \frac{1}{\left(\begin{array}{c}
2 j \\
j
\end{array}\right)} \frac{u^{j}}{j}\left(S_{2}^{2}-S_{4}\right) & =\frac{1}{60} \theta^{5} \tan \frac{\theta}{2},
\end{aligned}
$$

etc. Then, there are some cases $[16,30]$ when an arbitrary term of the $\varepsilon$-expansion can be calculated in terms of log-sine functions,

$$
\begin{aligned}
& { }_{2} F_{1}\left(\begin{array}{c|c}
1,1+\varepsilon \\
\frac{3}{2}
\end{array} \mid \sin ^{2} \frac{\theta}{2}\right)=\frac{\left(2 \cos \frac{\theta}{2}\right)^{-2 \varepsilon}}{\sin \theta} \sum_{j=0}^{\infty} \frac{(2 \varepsilon)^{j}}{j !}\left[\operatorname{Ls}_{j+1}(\pi-\theta)-\operatorname{Ls}_{j+1}(\pi)\right], \\
& { }_{2} F_{1}\left(\begin{array}{c}
1,1+\varepsilon \\
\frac{3}{2}+\varepsilon
\end{array} \mid \sin ^{2} \frac{\theta}{2}\right)=-\frac{(1+2 \varepsilon)}{\sin \theta\left(2 \sin \frac{\theta}{2}\right)^{2 \varepsilon}} \sum_{j=0}^{\infty} \frac{(2 \varepsilon)^{j}}{j !} \operatorname{Ls}_{j+1}(\theta), \\
& { }_{2} F_{1}\left(\begin{array}{c}
1,1+\varepsilon \\
\frac{3}{2}+\frac{1}{2} \varepsilon
\end{array} \mid \sin ^{2} \frac{\theta}{2}\right)=-\frac{1+\varepsilon}{(2 \sin \theta)^{1+\varepsilon}} \sum_{j=0}^{\infty} \frac{\varepsilon^{j}}{j !} \operatorname{Ls}_{j+1}(2 \theta) .
\end{aligned}
$$

Moreover, for a more general case an integral representation can be obtained [16,31],

$$
{ }_{2} F_{1}\left(\begin{array}{c}
1,1+a \varepsilon \\
\frac{3}{2}+b \varepsilon
\end{array} \mid \sin ^{2} \frac{\theta}{2}\right)=\frac{(1+2 b \varepsilon)\left(2 \cos \frac{\theta}{2}\right)^{2 b \varepsilon-2 a \varepsilon}}{\sin \theta\left(2 \sin \frac{\theta}{2}\right)^{2 b \varepsilon}} \int_{0}^{\theta} \mathrm{d} \phi\left(2 \sin \frac{\phi}{2}\right)^{2 b \varepsilon}\left(2 \cos \frac{\phi}{2}\right)^{2 a \varepsilon-2 b \varepsilon} .
$$

Any order of the $\varepsilon$-expansion of Eq. (2.13) can be expressed in terms of the generalized "log-sine-cosine" function $\operatorname{Lsc}_{i, j}(\theta)$ whose properties are listed in Appendix B of Ref. [16],

$$
\operatorname{Lsc}_{i, j}(\theta)=-\int_{0}^{\theta} \mathrm{d} \phi \ln ^{i-1}\left|2 \sin \frac{\phi}{2}\right| \ln ^{j-1}\left|2 \cos \frac{\phi}{2}\right| .
$$

Up to the level $i+j=5$, only one independent function appears, $\operatorname{Lsc}_{2,3}(\theta)$, that cannot be expressed in terms of ordinary log-sine integrals. Using the definition (2.14), one can obtain the following representation:

$$
\operatorname{Lsc}_{2,3}(\theta)=\frac{1}{12} \operatorname{Ls}_{4}(2 \theta)-\frac{1}{3} \operatorname{Ls}_{4}(\theta)-\frac{1}{6} \int_{0}^{\theta} \mathrm{d} \phi \ln ^{3}\left(\tan \frac{\phi}{2}\right) .
$$

The integral occurring in Eq. (2.15) can be expressed in terms of the inverse tangent integrals (see in Ref. [2]),

$$
\operatorname{Ti}_{N}(z)=\operatorname{Im}\left[\operatorname{Li}_{N}(\mathrm{i} z)\right]=\frac{1}{2 \mathrm{i}}\left[\operatorname{Li}_{N}(\mathrm{i} z)-\operatorname{Li}_{N}(-\mathrm{i} z)\right], \quad \operatorname{Ti}_{N}(z)=\int_{0}^{z} \frac{\mathrm{d} x}{x} \operatorname{Ti}_{N-1}(x)
$$


For $\operatorname{Ti}_{N}(z)$, the following integral representation (see, e.g., in Ref. [28]) is useful:

$$
\operatorname{Ti}_{N}(z)=\frac{(-1)^{N-1} z}{(N-1) !} \int_{0}^{1} \mathrm{~d} \xi \frac{\ln ^{N-1} \xi}{1+z^{2} \xi^{2}}
$$

In this way, we arrive at the following representation of $\operatorname{Lsc}_{2,3}(\theta)$ in terms of the inverse tangent integrals (2.16):

$$
\begin{aligned}
\operatorname{Lsc}_{2,3}(\theta)= & \frac{1}{12} \operatorname{Ls}_{4}(2 \theta)-\frac{1}{3} \mathrm{Ls}_{4}(\theta)+2 \mathrm{Ti}_{4}\left(\tan \frac{\theta}{2}\right)-2 \ln \left(\tan \frac{\theta}{2}\right) \mathrm{Ti}_{3}\left(\tan \frac{\theta}{2}\right) \\
& +\ln ^{2}\left(\tan \frac{\theta}{2}\right) \mathrm{Ti}_{2}\left(\tan \frac{\theta}{2}\right)-\frac{1}{6} \theta \ln ^{3}\left(\tan \frac{\theta}{2}\right) .
\end{aligned}
$$

Note that $\operatorname{Lsc}_{2,3}(\pi-\theta)$ can be reduced to $\operatorname{Lsc}_{2,3}(\theta)$ by using Eq. (A.27) of Ref. [16].

Using Eqs. (2.10) - (2.13), we obtain the following results for the weight-2 and weight-3 sums, in addition to Eq. (2.8):

$$
\begin{aligned}
& \sum_{j=1}^{\infty} \frac{1}{\left(\begin{array}{c}
2 j \\
j
\end{array}\right)} \frac{u^{j}}{j} S_{1}=2 \tan \frac{\theta}{2}\left[\operatorname{Ls}_{2}(\pi-\theta)-\theta L_{\theta}\right], \\
& \sum_{j=1}^{\infty} \frac{1}{\left(\begin{array}{c}
2 j \\
j
\end{array}\right)} \frac{u^{j}}{j} \bar{S}_{1}=\tan \frac{\theta}{2}\left[2 \operatorname{Ls}_{2}(\pi-\theta)+\operatorname{Ls}_{2}(\theta)+\theta l_{\theta}-2 \theta L_{\theta}\right], \\
& \sum_{j=1}^{\infty} \frac{1}{\left(\begin{array}{c}
2 j \\
j
\end{array}\right)} \frac{u^{j}}{j} S_{1}^{2}=4 \tan \frac{\theta}{2}\left[\operatorname{Ls}_{3}(\pi-\theta)-\operatorname{Ls}_{3}(\pi)-2 \operatorname{Ls}_{2}(\pi-\theta) L_{\theta}+\theta L_{\theta}^{2}+\frac{1}{24} \theta^{3}\right], \\
& \sum_{j=1}^{\infty} \frac{1}{\left(\begin{array}{c}
2 j \\
j
\end{array}\right)} \frac{u^{j}}{j} S_{1} \bar{S}_{1}=\tan \frac{\theta}{2}\left\{5\left[\operatorname{Ls}_{3}(\pi-\theta)-\operatorname{Ls}_{3}(\pi)\right]-\operatorname{Ls}_{3}(\theta)+\frac{1}{2} \operatorname{Ls}_{3}(2 \theta)-2 \operatorname{Ls}_{2}(\theta) L_{\theta}\right. \\
& \left.+2 \mathrm{Ls}_{2}(\pi-\theta) l_{\theta}-8 \mathrm{Ls}_{2}(\pi-\theta) L_{\theta}-2 \theta l_{\theta} L_{\theta}+4 \theta L_{\theta}^{2}+\frac{1}{12} \theta^{3}\right\} \text {, } \\
& \sum_{j=1}^{\infty} \frac{1}{\left(\begin{array}{c}
2 j \\
j
\end{array}\right)} \frac{u^{j}}{j}\left(\bar{S}_{2}+\bar{S}_{1}^{2}\right)=\tan \frac{\theta}{2}\left\{6\left[\operatorname{Ls}_{3}(\pi-\theta)-\operatorname{Ls}_{3}(\pi)\right]-3 \operatorname{Ls}_{3}(\theta)+4 \operatorname{Ls}_{2}(\pi-\theta) l_{\theta}\right. \\
& -8 \mathrm{Ls}_{2}(\pi-\theta) L_{\theta}+2 \mathrm{Ls}_{2}(\theta) l_{\theta}+\mathrm{Ls}_{3}(2 \theta)-4 \mathrm{Ls}_{2}(\theta) L_{\theta} \\
& \left.+\theta l_{\theta}^{2}-4 \theta L_{\theta} l_{\theta}+4 \theta L_{\theta}^{2}+\frac{1}{12} \theta^{3}\right\} \text {, }
\end{aligned}
$$

where $\operatorname{Ls}_{3}(\pi)=-\frac{1}{2} \pi \zeta_{2}$. In fact, all $\operatorname{Ls}_{j}(\pi)$ are expressible in terms of the $\zeta$-function [2].

For the weight 4 , the results for some combinations of sums involved in (2.4) can also be expressed in terms of log-sine functions, using Eqs. (2.10)-(2.13),

$$
\begin{aligned}
& \sum_{j=1}^{\infty} \frac{1}{\left(\begin{array}{c}
2 j \\
j
\end{array}\right)} \frac{u^{j}}{j} \mathcal{C}_{0}=8 \tan \frac{\theta}{2}\left\{\operatorname{Ls}_{4}(\pi-\theta)-\operatorname{Ls}_{4}(\pi)\right. \\
&\left.-3\left[\operatorname{Ls}_{3}(\pi-\theta)-\operatorname{Ls}_{3}(\pi)\right] L_{\theta}+3 \operatorname{Ls}_{2}(\pi-\theta) L_{\theta}^{2}-\theta L_{\theta}^{3}\right\} \\
& \sum_{j=1}^{\infty} \frac{1}{\left(\begin{array}{c}
2 j \\
j
\end{array}\right)} \frac{u^{j}}{j} \mathcal{C}_{1}=\tan \frac{\theta}{2}\left\{\frac{2}{3} \operatorname{Ls}_{4}(\theta)-\frac{1}{3} \operatorname{Ls}_{4}(2 \theta)-\frac{14}{3}\left[\operatorname{Ls}_{4}(\pi-\theta)-\mathrm{Ls}_{4}(\pi)\right]\right.
\end{aligned}
$$




$$
\begin{aligned}
& \quad+14\left[\mathrm{Ls}_{3}(\pi-\theta)-\mathrm{Ls}_{3}(\pi)\right] L_{\theta}-2 \mathrm{Ls}_{3}(\theta) l_{\theta}+\mathrm{Ls}_{3}(2 \theta)\left[L_{\theta}+l_{\theta}\right]-14 \mathrm{Ls}_{2}(\pi-\theta) L_{\theta}^{2} \\
& \left.\quad+2 \mathrm{Ls}_{2}(\pi-\theta) l_{\theta}^{2}-2 \mathrm{Ls}_{2}(2 \theta) L_{\theta} l_{\theta}-\mathrm{Ls}_{2}(2 \theta) L_{\theta}^{2}-2 \theta L_{\theta} l_{\theta}^{2}-2 \theta L_{\theta}^{2} l_{\theta}+4 \theta L_{\theta}^{3}\right\}, \\
& \sum_{j=1}^{\infty} \frac{1}{\left(\begin{array}{c}
2 j \\
j
\end{array}\right)} \frac{u^{j}}{j} \mathcal{C}_{2}=\tan \frac{\theta}{2}\left\{\frac{1}{3} \mathrm{Ls}_{4}(2 \theta)-\mathrm{Ls}_{4}(\theta)+4\left[\mathrm{Ls}_{4}(\pi-\theta)-\mathrm{Ls}_{4}(\pi)\right]-\mathrm{Ls}_{3}(2 \theta)\left(l_{\theta}+L_{\theta}\right)\right. \\
& \quad-12\left[\mathrm{Ls}_{3}(\pi-\theta)-\mathrm{Ls}_{3}(\pi)\right] L_{\theta}+3 \mathrm{Ls}_{3}(\theta) l_{\theta}+12 \mathrm{Ls}_{2}(\pi-\theta) L_{\theta}^{2}-3 \mathrm{Ls}_{2}(\pi-\theta) l_{\theta}^{2} \\
& \left.\quad-\frac{1}{2} \mathrm{Ls}_{2}(2 \theta) l_{\theta}^{2}+\mathrm{Ls}_{2}(2 \theta) L_{\theta}^{2}+2 \mathrm{Ls}_{2}(2 \theta) l_{\theta} L_{\theta}+2 \theta l_{\theta}^{2} L_{\theta}+2 \theta l_{\theta} L_{\theta}^{2}-\frac{1}{3} \theta l_{\theta}^{3}-\frac{10}{3} \theta L_{\theta}^{3}\right\},(2.26
\end{aligned}
$$

where $\operatorname{Ls}_{4}(\pi)=\frac{3}{2} \pi \zeta_{3}$ and the combinations of the harmonic sums $C_{j}$ are defined in (2.5). Finally, using (2.13) the result for the sum involving $C_{3}$ can be expressed in terms of the Lsc-function (2.18),

$$
\begin{aligned}
& \sum_{j=1}^{\infty} \frac{1}{\left(\begin{array}{c}
2 j \\
j
\end{array}\right)} \frac{u^{j}}{j} \mathcal{C}_{3}=-4 \tan \frac{\theta}{2}\left\{2 \operatorname{Lsc}_{2,3}(\theta)+2\left[\operatorname{Ls}_{4}(\pi-\theta)-\mathrm{Ls}_{4}(\pi)\right]\right. \\
& \quad+2\left[\mathrm{Ls}_{3}(\pi-\theta)-\mathrm{Ls}_{3}(\pi)\right] l_{\theta}-8\left[\mathrm{Ls}_{3}(\pi-\theta)-\mathrm{Ls}_{3}(\pi)\right] L_{\theta}-\mathrm{Ls}_{3}(2 \theta) L_{\theta}+2 \mathrm{Ls}_{3}(\theta) L_{\theta} \\
& \left.\quad+8 \mathrm{Ls}_{2}(\pi-\theta) L_{\theta}^{2}-4 \mathrm{Ls}_{2}(\pi-\theta) L_{\theta} l_{\theta}+\mathrm{Ls}_{2}(2 \theta) L_{\theta}^{2}-2 \theta L_{\theta}^{3}+2 \theta L_{\theta}^{2} l_{\theta}\right\} .
\end{aligned}
$$

Some of the results (2.19) -(2.27) can be written in a slightly different form by means of relations [2]

$$
\mathrm{Ls}_{2}(\theta)=\mathrm{Cl}_{2}(\theta), \quad \mathrm{Cl}_{2 n}(\pi+\theta)=-\mathrm{Cl}_{2 n}(\pi-\theta), \quad \mathrm{Cl}_{2 n+1}(\pi+\theta)=\mathrm{Cl}_{2 n+1}(\pi-\theta) .
$$

However, in this way one cannot obtain results for the two remaining combinations in (2.4), involving $A_{1}^{2}-A_{2}=2 a_{1} a_{2}$, since in Eq. (2.13) we always have $a_{1} a_{2}=0$. An interesting relation between these two functions is obtained in Appendix B. It should also be noted that for higher hypergeometric functions other combinations of sums may arise at this level (see below).

\subsection{Expansion of higher functions}

Let us consider the hypergeometric function of the following type:

${ }_{P+1} F_{P}\left(\begin{array}{c}\frac{3}{2}+b_{1} \varepsilon, \ldots, \frac{3}{2}+b_{J-1} \varepsilon, 1+a_{1} \varepsilon, \ldots, 1+a_{K} \varepsilon, 2+d_{1} \varepsilon \ldots, 2+d_{L} \varepsilon \\ \frac{3}{2}+f_{1} \varepsilon, \ldots, \frac{3}{2}+f_{J} \varepsilon, 1+e_{1} \varepsilon, \ldots, 1+e_{R} \varepsilon, 2+c_{1} \varepsilon, \ldots, 2+c_{K+L-R-2} \varepsilon\end{array} \mid z\right)$,

where $P=K+L+J-2$. Using the representation (2.2) its $\varepsilon$-expansion can be reduced to inverse binomial sums (1.1). The original hypergeometric function (2.28) can be written as (see details in Appendix B of Ref. [16])

$$
\begin{aligned}
{ }_{P+1} F_{P}\left(\begin{array}{c}
\left\{\frac{3}{2}+b_{i} \varepsilon\right\}^{J-1},\left\{1+a_{i} \varepsilon\right\}^{K},\left\{2+d_{i} \varepsilon\right\}^{L} \\
\left\{\frac{3}{2}+f_{i} \varepsilon\right\}^{J},\left\{1+e_{i} \varepsilon\right\}^{R},\left\{2+c_{i} \varepsilon\right\}^{K+L-R-2}
\end{array} \mid z\right) \\
=\frac{2}{u} \frac{\prod_{s=1}^{K+L-R-2}\left(1+c_{s} \varepsilon\right) \prod_{k=1}^{J}\left(1+2 f_{k} \varepsilon\right)}{\prod_{i=1}^{L}\left(1+d_{i} \varepsilon\right) \Pi_{r=1}^{J-1}\left(1+2 b_{r} \varepsilon\right)} \sum_{j=1}^{\infty} \frac{1}{\left(\begin{array}{c}
2 j \\
j
\end{array}\right)} \frac{u^{j}}{j^{K-R-1}} \Delta,
\end{aligned}
$$


where $u=4 z$,

$$
\begin{aligned}
\Delta= & \exp \left[\sum_{k=1}^{\infty} \frac{(-\varepsilon)^{k}}{k}\left(S_{k} T_{k}+2^{k} U_{k} \bar{S}_{k}+W_{k} j^{-k}\right)\right] \\
= & 1-\varepsilon\left(W_{1} j^{-1}+T_{1} S_{1}+2 U_{1} \bar{S}_{1}\right)+\varepsilon^{2}\left[\frac{1}{2} j^{-2}\left(W_{2}+W_{1}^{2}\right)+W_{1} j^{-1}\left(T_{1} S_{1}+2 U_{1} \bar{S}_{1}\right)\right. \\
& \left.+2 T_{1} U_{1} S_{1} \bar{S}_{1}+\frac{1}{2} T_{2} S_{2}+\frac{1}{2} T_{1}^{2} S_{1}^{2}+2\left(U_{2} \bar{S}_{2}+U_{1}^{2} \bar{S}_{1}^{2}\right)\right] \\
& -\varepsilon^{3}\left\{\frac{1}{6} j^{-3}\left(2 W_{3}+3 W_{1} W_{2}+W_{1}^{3}\right)+\frac{1}{2} j^{-2}\left(W_{2}+W_{1}^{2}\right)\left(T_{1} S_{1}+2 U_{1} \bar{S}_{1}\right)\right. \\
& +\frac{1}{2} W_{1} j^{-1}\left[T_{2} S_{2}+T_{1}^{2} S_{1}^{2}+4 T_{1} U_{1} S_{1} \bar{S}_{1}+4\left(U_{2} \bar{S}_{2}+U_{1}^{2} \bar{S}_{1}^{2}\right)\right] \\
& +\frac{1}{6} T_{1}^{3} S_{1}^{3}+T_{1}^{2} U_{1} S_{1}^{2} \bar{S}_{1}+\frac{1}{2} T_{1} T_{2} S_{1} S_{2}+T_{2} U_{1} S_{2} \bar{S}_{1}+\frac{1}{3} T_{3} S_{3} \\
& \left.+2 T_{1} S_{1}\left(U_{2} \bar{S}_{2}+U_{1}^{2} \bar{S}_{1}^{2}\right)+\frac{4}{3}\left(U_{1}^{3} \bar{S}_{1}^{3}+3 U_{1} U_{2} \bar{S}_{1} \bar{S}_{2}+2 U_{3} \bar{S}_{3}\right)\right\}+\mathcal{O}\left(\varepsilon^{4}\right)
\end{aligned}
$$

and we introduced the constants

$$
\begin{aligned}
& A_{k} \equiv \sum a_{i}^{k}, \quad B_{k} \equiv \sum b_{i}^{k}, \quad C_{k} \equiv \sum c_{i}^{k}, \quad D_{k} \equiv \sum d_{i}^{k}, \quad E_{k} \equiv \sum e_{i}^{k}, \quad F_{k} \equiv \sum f_{i}^{k}, \\
& T_{k} \equiv B_{k}+C_{k}+E_{k}-A_{k}-D_{k}-F_{k}, \quad U_{k} \equiv F_{k}-B_{k}, \quad W_{k} \equiv C_{k}-D_{k},
\end{aligned}
$$

where the summations extend over all possible values of the parameters in Eq. (2.28). We can see that for the general values of the parameters of the ${ }_{P+1} F_{P}$ function (2.28) we need all the occurring sums separately. However, for many applications, it is sufficient to consider the case $J=1$ only. In this case, $B_{k}=0$ and $U_{k}=F_{k}=f_{1}^{k}$. Substituting this into Eq. (2.30), we see that $\bar{S}_{2}$ and $\bar{S}_{3}$ would only appear in combinations $\left(\bar{S}_{2}+\bar{S}_{1}^{2}\right)$ and $\left(\bar{S}_{1}^{3}+3 \bar{S}_{1} \bar{S}_{2}+2 \bar{S}_{3}\right)$. Furthermore, using the notations (2.5), Eq. (2.30) in the case $J=1$ can be presented as

$$
\begin{aligned}
\left.\Delta\right|_{J=1}= & 1-\varepsilon\left(W_{1} j^{-1}+T_{1} S_{1}+2 f_{1} \bar{S}_{1}\right)+\varepsilon^{2}\left[\frac{1}{2} j^{-2}\left(W_{2}+W_{1}^{2}\right)+W_{1} j^{-1}\left(T_{1} S_{1}+2 f_{1} \bar{S}_{1}\right)\right. \\
& \left.+2 f_{1} T_{1} S_{1} \bar{S}_{1}+\frac{1}{2} T_{2} S_{2}+\frac{1}{2} T_{1}^{2} S_{1}^{2}+2 f_{1}^{2}\left(\bar{S}_{2}+\bar{S}_{1}^{2}\right)\right] \\
& -\varepsilon^{3}\left\{\frac{1}{6} j^{-3}\left(2 W_{3}+3 W_{1} W_{2}+W_{1}^{3}\right)+\frac{1}{2} j^{-2}\left(W_{2}+W_{1}^{2}\right)\left(T_{1} S_{1}+2 f_{1} \bar{S}_{1}\right)\right. \\
& +\frac{1}{2} W_{1} j^{-1}\left[T_{2} S_{2}+T_{1}^{2} S_{1}^{2}+4 f_{1} T_{1} S_{1} \bar{S}_{1}+4 f_{1}^{2}\left(\bar{S}_{2}+\bar{S}_{1}^{2}\right)\right] \\
& +\frac{1}{6} T_{1}\left(T_{1}^{2}+3 f_{1} T_{1}+3 f_{1}^{2}\right) S_{1}^{3}+\frac{1}{2} T_{1}\left(T_{2}-f_{1} T_{1}-f_{1}^{2}\right) S_{1} S_{2}+\frac{1}{3} T_{3} S_{3} \\
& +f_{1}\left(T_{2}+T_{1}^{2}+2 f_{1} T_{1}+2 f_{1}^{2}\right) S_{2} \bar{S}_{1}+2 f_{1}^{2} T_{1} \mathcal{C}_{1}-4 f_{1}^{3} \mathcal{C}_{2} \\
& \left.-\frac{1}{2} f_{1}\left(T_{1}+2 f_{1}\right)\left(T_{1}+3 f_{1}\right) \mathcal{C}_{3}\right\}+\mathcal{O}\left(\varepsilon^{4}\right) .
\end{aligned}
$$

Eq. (2.30) also shows that we may need the sums with higher values of $c$ (the power of $1 / j$ ), which would come from $1 / j^{K-R-1}$ in Eq. (2.29) and extra powers of $1 / j$ in Eq. (2.30). Note that in the case $K-R=2$, when we have the same number of $c_{i}$ and $d_{i}$, we have $1 / j$ factor in the sum in Eq. (2.29). Moreover, when the parameters $\left\{2+c_{i}\right\}$ and $\left\{2+d_{i}\right\}$ are at all missing, we get no extra factors of $1 / j$ in Eq. (2.30), for all $W_{k}$ would vanish.

For the sums of the type (1.1) with an arbitrary integer power $c \geq 2$ and $0 \leq u \leq 4$, the 
following one-fold integral representation [27] is useful:

$$
\sum_{j=1}^{\infty} \frac{u^{j}}{j^{c}} \frac{f(j)}{\left(\begin{array}{c}
2 j \\
j
\end{array}\right)}=\frac{1}{(c-2) !} \int_{0}^{\theta} \mathrm{d} \phi \frac{\cos \frac{\phi}{2}}{\sin \frac{\phi}{2}}\left[\ln u-2 \ln \left(2 \sin \frac{\phi}{2}\right)\right]^{c-2} \sum_{j=1}^{\infty} \frac{\left(4 \sin ^{2} \frac{\phi}{2}\right)^{j}}{j} \frac{f(j)}{\left(\begin{array}{c}
2 j \\
j
\end{array}\right)}
$$

where $f(j)$ stands for an arbitrary combination of the harmonic sums. In this manner, the original sum of the type (1.1) with an arbitrary positive integer parameter $c \geq 2$ is reduced to a one-fold integral representation containing a sum with $c=1$ in the integrand. Using the representation (2.32) we can generalize our results (2.8), (2.9), (2.19) $-(2.27)$ to the case of an arbitrary integer $c>1$. However, only for special types of sums these results are expressible in terms of generalized log-sine functions (1.4):

$$
\begin{aligned}
& \sum_{j=1}^{\infty} \frac{u^{j}}{j^{c}} \frac{1}{\left(\begin{array}{c}
2 j \\
j
\end{array}\right)} S_{2}=-\frac{1}{6} \sum_{i=0}^{c-2} \frac{(-2)^{i}}{i !(c-2-i) !}(\ln u)^{c-2-i} \operatorname{Ls}_{i+4}^{(3)}(\theta) \\
& \sum_{j=1}^{\infty} \frac{u^{j}}{j^{c}} \frac{1}{\left(\begin{array}{c}
2 j \\
j
\end{array}\right)}\left(S_{2}^{2}-S_{4}\right)=-\frac{1}{60} \sum_{i=0}^{c-2} \frac{(-2)^{i}}{i !(c-2-i) !}(\ln u)^{c-2-i} \operatorname{Ls}_{i+6}^{(5)}(\theta)
\end{aligned}
$$

where $c \geq 2,0 \leq u \leq 4$, and the angle $\theta$ is defined in Eq. (1.3).

Further results can be extracted from integral representation (2.32) by using the integration rules for Clausen's function [2] and the following relation:

$$
k \int_{0}^{\theta} \mathrm{d} \phi \phi^{k-1} \operatorname{Ls}_{j}^{(i)}(m \phi)=\theta^{k} \operatorname{Ls}_{j}^{(i)}(m \theta)-\frac{1}{m^{k}} \operatorname{Ls}_{j+k}^{(i+k)}(m \theta) .
$$

In this way, we obtain

$$
\begin{aligned}
& \sum_{j=1}^{\infty} \frac{1}{\left(\begin{array}{c}
2 j \\
j
\end{array}\right)} \frac{u^{j}}{j^{2}} S_{1}=4 \mathrm{Cl}_{3}(\pi-\theta)-2 \theta \mathrm{Cl}_{2}(\pi-\theta)+3 \zeta_{3}, \\
& \sum_{j=1}^{\infty} \frac{1}{\left(\begin{array}{c}
2 j \\
j
\end{array}\right)} \frac{u^{j}}{j^{2}} \bar{S}_{1}=-2 \mathrm{Cl}_{3}(\theta)+4 \mathrm{Cl}_{3}(\pi-\theta)-2 \theta \mathrm{Cl}_{2}(\pi-\theta)-\theta \mathrm{Cl}_{2}(\theta)+5 \zeta_{3} \\
& \sum_{j=1}^{\infty} \frac{1}{\left(\begin{array}{c}
2 j \\
j
\end{array}\right)} \frac{u^{j}}{j^{2}} S_{1}^{2}=4 \theta\left[\operatorname{Ls}_{3}(\pi-\theta)-\operatorname{Ls}_{3}(\pi)\right]-4\left[\operatorname{Ls}_{2}(\pi-\theta)\right]^{2}+\frac{1}{24} \theta^{4}, \\
& \sum_{j=1}^{\infty} \frac{1}{\left(\begin{array}{c}
2 j \\
j
\end{array}\right)} \frac{u^{j}}{j^{2}} S_{1} \bar{S}_{1}=5 \theta\left[\operatorname{Ls}_{3}(\pi-\theta)-\operatorname{Ls}_{3}(\pi)\right]-\theta \operatorname{Ls}_{3}(\theta)+\frac{1}{2} \theta \operatorname{Ls}_{3}(2 \theta) \\
& -4\left[\operatorname{Ls}_{2}(\pi-\theta)\right]^{2}+\frac{1}{48} \theta^{4}-2 \operatorname{Ls}_{2}(\pi-\theta) \operatorname{Ls}_{2}(\theta), \\
& \sum_{j=1}^{\infty} \frac{1}{\left(\begin{array}{c}
2 j \\
j
\end{array}\right)} \frac{u^{j}}{j^{2}}\left(\bar{S}_{1}^{2}+\bar{S}_{2}\right)=6 \theta\left[\operatorname{Ls}_{3}(\pi-\theta)-\operatorname{Ls}_{3}(\pi)\right]-3 \theta \operatorname{Ls}_{3}(\theta)+\theta \operatorname{Ls}_{3}(2 \theta) \\
& -4\left[\operatorname{Ls}_{2}(\pi-\theta)\right]^{2}-\left[\operatorname{Ls}_{2}(\theta)\right]^{2}+\frac{1}{48} \theta^{4}-4 \operatorname{Ls}_{2}(\pi-\theta) \operatorname{Ls}_{2}(\theta)
\end{aligned}
$$


However, other sums are not expressible in terms of the generalized log-sine functions. One needs to introduce a new function ${ }^{4}$,

$$
\Phi(\theta) \equiv \int_{0}^{\theta} \mathrm{d} \phi \operatorname{Ls}_{2}(\phi) \ln \left(2 \cos \frac{\phi}{2}\right),
$$

which obeys the following symmetry property:

$$
\Phi(\theta)+\Phi(\pi-\theta)=\Phi(\pi)+\operatorname{Ls}_{2}(\pi-\theta) \operatorname{Ls}_{2}(\theta)
$$

where

$$
\Phi(\pi)=\frac{1}{6} \ln ^{4} 2-\zeta_{2} \ln ^{2} 2+\frac{7}{2} \zeta_{3} \ln 2-\frac{53}{16} \zeta_{4}+4 \operatorname{Li}_{4}\left(\frac{1}{2}\right)=0.64909 \ldots .
$$

The following sums of the weight 4 are expressible in terms of the function $\Phi(\theta)$ :

$$
\begin{gathered}
\sum_{j=1}^{\infty} \frac{1}{\left(\begin{array}{c}
2 j \\
j
\end{array}\right)} \frac{u^{j}}{j^{3}} S_{1}=2\left[\mathrm{Ls}_{4}^{(1)}(\pi-\theta)-\mathrm{Ls}_{4}^{(1)}(\pi)\right]-\frac{1}{2} \mathrm{Ls}_{4}^{(1)}(2 \theta)+2 \mathrm{Ls}_{4}^{(1)}(\theta)-4 \theta \mathrm{Ls}_{2}(\pi-\theta) l_{\theta} \\
\quad-2 \pi\left[\mathrm{Ls}_{3}(\pi-\theta)-\mathrm{Ls}_{3}(\pi)\right]+8\left[\mathrm{Cl}_{3}(\pi-\theta)-\mathrm{Cl}_{3}(\pi)\right] l_{\theta}+4 \Phi(\pi-\theta)-4 \Phi(\pi),(2 . \\
\sum_{j=1}^{\infty} \frac{1}{\left(\begin{array}{c}
2 j \\
j
\end{array}\right)} \frac{u^{j}}{j^{3}} \bar{S}_{1}=2\left[\mathrm{Ls}_{4}^{(1)}(\pi-\theta)-\mathrm{Ls}_{4}^{(1)}(\pi)\right]-\frac{1}{2} \mathrm{Ls}_{4}^{(1)}(2 \theta)+4 \mathrm{Ls}_{4}^{(1)}(\theta)+\left[\mathrm{Ls}_{2}(\theta)\right]^{2} \\
\quad-2 \pi\left[\mathrm{Ls}_{3}(\pi-\theta)-\mathrm{Ls}_{3}(\pi)\right]+8\left[\mathrm{Cl}_{3}(\pi-\theta)-\mathrm{Cl}_{3}(\pi)\right] l_{\theta}-4\left[\mathrm{Cl}_{3}(\theta)-\zeta_{3}\right] l_{\theta} \\
\quad-2 \theta \mathrm{Ls}_{2}(\theta) l_{\theta}-4 \theta \mathrm{Ls}_{2}(\pi-\theta) l_{\theta}+4 \Phi(\pi-\theta)-4 \Phi(\pi),
\end{gathered}
$$

where we have also used the following integral:

$$
\begin{aligned}
\int_{0}^{\theta} \mathrm{d} \phi \phi \ln \left(2 \cos \frac{\phi}{2}\right) \ln \left(2 \sin \frac{\phi}{2}\right)= & -\frac{1}{8} \operatorname{Ls}_{4}^{(1)}(2 \theta)+\frac{1}{2} \operatorname{Ls}_{4}^{(1)}(\theta)+\frac{1}{2}\left[\operatorname{Ls}_{4}^{(1)}(\pi-\theta)-\operatorname{Ls}_{4}^{(1)}(\pi)\right] \\
& -\frac{1}{2} \pi\left[\operatorname{Ls}_{3}(\pi-\theta)-\operatorname{Ls}_{3}(\pi)\right] .
\end{aligned}
$$

Let us note that $\Phi(\theta)$ can be related to the real part of a certain harmonic polylogarithm [4] of complex argument,

$$
\Phi(\theta)=\frac{1}{96} \theta^{2}(2 \pi-\theta)^{2}-L_{\theta} \mathrm{Cl}_{3}(\theta)+\zeta_{3} \ln 2-\mathrm{H}_{-1,0,0,1}(1)+\frac{1}{2}\left[\mathrm{H}_{-1,0,0,1}\left(e^{\mathrm{i} \theta}\right)+\mathrm{H}_{-1,0,0,1}\left(e^{-\mathrm{i} \theta}\right)\right],
$$

where (for details, see Appendix A)

$$
\begin{aligned}
& \mathrm{H}_{-1,0,0,1}(y)=\int_{0}^{y} \mathrm{~d} x \frac{\mathrm{Li}_{3}(x)}{1+x}, \\
& \mathrm{H}_{-1,0,0,1}(1)=-\frac{1}{12} \ln ^{4} 2+\frac{1}{2} \zeta_{2} \ln ^{2} 2-\frac{3}{4} \zeta_{3} \ln 2+\frac{3}{2} \zeta_{4}-2 \mathrm{Li}_{4}\left(\frac{1}{2}\right)=0.33955 \ldots .
\end{aligned}
$$

The function $\mathrm{H}_{-1,0,0,1}(y)$ has a branch cut starting at the point $y=-1(\theta= \pm \pi)$, which is subtracted by logarithmic terms in (2.45), so that $\Phi(\theta)$ is a smooth function of variable $\theta$.

\footnotetext{
${ }^{4}$ Alternatively, instead of $\Phi(\theta)$ one may introduce the generalized Glashier function $\mathrm{Gl}_{4}(\theta ; 1)$, see Eq. A.16) in Appendix A.
} 


\subsection{Further results for the sums}

For further investigation of the sums of the type (1.1), a recursive approach appears to be useful. Some ideas applied below are described in the book [32]. Let us rewrite Eq. (1.1) in the following form:

$$
\Sigma_{a_{1}, \ldots, a_{p} ; b_{1}, \ldots, b_{q} ; c}^{i_{1}, \ldots, i_{p} ; j_{1}, \ldots, j_{q}}(u) \equiv \Sigma_{A ; B ; c}(u)=\sum_{j=1}^{\infty} u^{j} \eta_{A ; B ; c}(j)
$$

where $A \equiv\left(\begin{array}{l}i_{1}, \ldots, i_{p} \\ a_{1}, \ldots, a_{p}\end{array}\right)$ and $B \equiv\left(\begin{array}{l}j_{1}, \ldots, j_{q} \\ b_{1}, \ldots, b_{q}\end{array}\right)$ denote the collective sets of indices, whereas $\eta_{A ; B ; c}(j)$ is the coefficient of $u^{j}$ in Eq. (1.1), i.e. the product of binomial sums divided by $\left(\begin{array}{c}2 j \\ j\end{array}\right) j^{c}$.

The idea is to find a recurrence relation ${ }^{5}$, with respect to $j$, for the coefficients $\eta_{A ; B ; c}(j)$, and then transform it into a differential equation for the generating function $\Sigma_{A ; B ; c}(u)$. In this way, the problem of summing the series would be reduced to solving a differential equation. Using the explicit form of $\eta_{A ; B ; c}(j)$ given in Eq. (1.1), the recurrence relation can be written in the following form:

$$
2(2 j+1)(j+1)^{c-1} \eta_{A ; B ; c}(j+1)-j^{c} \eta_{A ; B ; c}(j)=r_{A ; B}(j),
$$

where the explicit form of the "remainder" $r_{A ; B}(j)$ is given by

$$
\begin{aligned}
\left(\begin{array}{c}
2 j \\
j
\end{array}\right) r_{A ; B}(j)= & \prod_{k=1}^{p}\left[S_{a_{k}}(j-1)+j^{-a_{k}}\right]^{i_{k}} \prod_{l=1}^{q}\left[S_{b_{l}}(2 j-1)+(2 j)^{-b_{l}}+(2 j+1)^{-b_{l}}\right]^{j_{l}} \\
& -\prod_{k=1}^{p}\left[S_{a_{k}}(j-1)\right]^{i_{k}} \prod_{l=1}^{q}\left[S_{b_{l}}(2 j-1)\right]^{j_{l}}
\end{aligned}
$$

In other words, it contains all contributions generated by $j^{-a_{k}},(2 j)^{-b_{l}}$ and $(2 j+1)^{-b_{l}}$ which appear because of the shift of the index $j$.

Multiplying both sides of Eq. (2.49) by $u^{j}$, summing from 1 to infinity, and using the fact that any extra power of $j$ corresponds to the derivative $u(\mathrm{~d} / \mathrm{d} u)$, we arrive at the following differential equation for the generating function $\Sigma_{A ; B ; c}(u)$ :

$$
\left(\frac{4}{u}-1\right)\left(u \frac{\mathrm{d}}{\mathrm{d} u}\right)^{c} \Sigma_{A ; B ; c}(u)-\frac{2}{u}\left(u \frac{\mathrm{d}}{\mathrm{d} u}\right)^{c-1} \Sigma_{A ; B ; c}(u)=2 \eta_{A ; B ; c}(1)+R_{A ; B}(u),
$$

where $\eta_{A ; B ; c}(1)=\frac{1}{2} \delta_{p 0}$ and $R_{A ; B}(u) \equiv \sum_{j=1}^{\infty} u^{j} r_{A ; B}(j)$. Using Eq. (2.50) we obtain

$$
\begin{aligned}
R_{a_{1} ;-}(u) & =\sum_{j=1}^{\infty} \frac{u^{j}}{\left(\begin{array}{c}
2 j \\
j
\end{array}\right)} \frac{1}{j^{a_{1}}}, \\
R_{-; b_{1}}(u) & =\sum_{j=1}^{\infty} \frac{u^{j}}{\left(\begin{array}{c}
2 j \\
j
\end{array}\right)}\left[\frac{1}{(2 j)^{b_{1}}}+\frac{1}{(2 j+1)^{b_{1}}}\right],
\end{aligned}
$$

\footnotetext{
${ }^{5}$ About application of the difference equations in the physical calculations we refer to [33].
} 


$$
\begin{aligned}
& R_{a_{1}, a_{2} ;-}(u)=\sum_{j=1}^{\infty} \frac{u^{j}}{\left(\begin{array}{c}
2 j \\
j
\end{array}\right)}\left[\frac{S_{a_{1}}}{j^{a_{2}}}+\frac{S_{a_{2}}}{j^{a_{1}}}+\frac{1}{j^{a_{1}+a_{2}}}\right], \\
& R_{a_{1} ; b_{1}}(u)=\sum_{j=1}^{\infty} \frac{u^{j}}{\left(\begin{array}{c}
2 j \\
j
\end{array}\right)}\left[\frac{S_{a_{1}}}{(2 j)^{b_{1}}}+\frac{S_{a_{1}}}{(2 j+1)^{b_{1}}}+\frac{\bar{S}_{b_{1}}}{j^{a_{1}}}+\frac{1}{j^{a_{1}}(2 j)^{b_{1}}}+\frac{1}{j^{a_{1}}(2 j+1)^{b_{1}}}\right], \\
& R_{-; b_{1}, b_{2}}(u)=\sum_{j=1}^{\infty} \frac{u^{j}}{\left(\begin{array}{c}
2 j \\
j
\end{array}\right)}\left[\frac{\bar{S}_{b_{1}}}{(2 j)^{b_{2}}}+\frac{\bar{S}_{b_{2}}}{(2 j)^{b_{1}}}+\frac{\bar{S}_{b_{1}}}{(2 j+1)^{b_{2}}}+\frac{\bar{S}_{b_{2}}}{(2 j+1)^{b_{1}}}+\frac{1}{(2 j)^{b_{1}+b_{2}}}\right. \\
& \left.+\frac{1}{j^{b_{1}}(2 j+1)^{b_{2}}}+\frac{1}{j^{b_{2}}(2 j+1)^{b_{1}}}+\frac{1}{(2 j+1)^{b_{1}+b_{2}}}\right] \text {, } \\
& R_{a_{1}, a_{2}, a_{3} ;-}(u)=\sum_{j=1}^{\infty} \frac{u^{j}}{\left(\begin{array}{c}
2 j \\
j
\end{array}\right)}\left[\frac{S_{a_{1}} S_{a_{2}}}{j^{a_{3}}}+\frac{S_{a_{1}} S_{a_{3}}}{j^{a_{2}}}+\frac{S_{a_{2}} S_{a_{3}}}{j^{a_{1}}}+\frac{S_{a_{1}}}{j^{a_{2}+a_{3}}}+\frac{S_{a_{2}}}{j^{a_{1}+a_{3}}}+\frac{S_{a_{3}}}{j^{a_{1}+a_{2}}}+\frac{1}{j^{a_{1}+a_{2}+a_{3}}}\right] .
\end{aligned}
$$

In terms of the geometrical variable (1.3),

$$
u \equiv u_{\theta}=4 \sin ^{2} \frac{\theta}{2}, \quad \frac{4}{u}-1=\cot ^{2} \frac{\theta}{2}, \quad u \frac{\mathrm{d}}{\mathrm{d} u}=\tan \frac{\theta}{2} \frac{\mathrm{d}}{\mathrm{d} \theta},
$$

the differential equation (2.51) takes the following form:

$$
\frac{1}{2 \sin ^{2} \frac{\theta}{2}}\left(2 \sin \frac{\theta}{2} \cos \frac{\theta}{2} \frac{\mathrm{d}}{\mathrm{d} \theta}-1\right)\left(\tan \frac{\theta}{2} \frac{\mathrm{d}}{\mathrm{d} \theta}\right)^{c-1} \Sigma_{A ; B ; c}\left(u_{\theta}\right)=\delta_{p 0}+R_{A, B}\left(u_{\theta}\right) .
$$

Furthermore, Eq. (2.59) can be represented as

$$
\left(\tan \frac{\theta}{2} \frac{\mathrm{d}}{\mathrm{d} \theta}\right)^{c-1} \Sigma_{A ; B ; c}\left(u_{\theta}\right)=\tan \frac{\theta}{2} \sigma_{A ; B}(\theta)
$$

where

$$
\frac{\mathrm{d}}{\mathrm{d} \theta} \sigma_{A ; B}(\theta)=\delta_{p 0}+R_{A, B}\left(u_{\theta}\right), \quad \sigma_{A ; B}(\theta)=\delta_{p 0} \theta+\int_{0}^{\theta} \mathrm{d} \phi R_{A ; B}\left(u_{\phi}\right),
$$

with $u_{\phi}=4 \sin ^{2} \frac{\phi}{2}$. In particular, for $c=1$ and $c=2$ we have, respectively,

$$
\begin{aligned}
& \Sigma_{A ; B ; 1}\left(u_{\theta}\right)=\tan \frac{\theta}{2} \sigma_{A ; B}(\theta)=\tan \frac{\theta}{2}\left[\delta_{p 0} \theta+\int_{0}^{\theta} \mathrm{d} \phi R_{A ; B}\left(u_{\phi}\right)\right], \\
& \Sigma_{A ; B ; 2}\left(u_{\theta}\right)=\int_{0}^{\theta} \mathrm{d} \phi \sigma_{A ; B}(\phi)=\theta \sigma_{A ; B}(\theta)-\frac{1}{2} \delta_{p 0} \theta^{2}-\int_{0}^{\theta} \phi \mathrm{d} \phi R_{A, B}\left(u_{\phi}\right) .
\end{aligned}
$$

Introducing $l_{\theta} \equiv \ln \left(2 \sin \frac{\theta}{2}\right)=\frac{1}{2} \ln u_{\theta}$, Eq. (2.60) can be rewritten as

$$
\left(\frac{1}{2} \frac{\mathrm{d}}{\mathrm{d} l_{\theta}}\right)^{c-k} \Sigma_{A ; B ; c}\left(u_{\theta}\right)=\Sigma_{A ; B ; k}\left(u_{\theta}\right)
$$


which is nothing but the differential form of the relation (2.32). The iterative solution of Eq. (2.64) is

$$
\Sigma_{A ; B ; c}\left(u_{\theta}\right)=-\sum_{i=1}^{k} \frac{(-2)^{i}}{i !} l_{\theta}^{i} \Sigma_{A ; B ; c-i}\left(u_{\theta}\right)+\frac{(-2)^{k}}{k !} \int_{0}^{\theta} \mathrm{d} \phi l_{\phi}^{k} \frac{\mathrm{d} \Sigma_{A ; B ; c-k}\left(u_{\phi}\right)}{\mathrm{d} \phi},
$$

where $l_{\phi} \equiv \ln \left(2 \sin \frac{\phi}{2}\right)=\frac{1}{2} \ln u_{\phi}$.

In particular, this solution allows us to formulate and prove the following important statement. If for some $k$ the derivative $\Sigma_{A ; B ; c-k}\left(u_{\theta}\right)$ is expressible only in terms of the powers of $\theta$ and $l_{\theta}$, then the sum $\Sigma_{A ; B ; c}\left(u_{\theta}\right)$ can be presented in terms of the generalized log-sine functions. Moreover, according to a statement proven in Appendix A.1 of Ref. [16], the analytic continuation of any generalized log-sine function $\operatorname{Ls}_{j}^{(k)}(\theta)$ can be expressed in terms of Nielsen polylogarithms.

Let us now explain how this general method works for specific sums of interest. Consider

$$
\Sigma_{3 ;-; 1}^{1 ;-}(u)=\sum_{j=1}^{\infty} \frac{1}{\left(\begin{array}{c}
2 j \\
j
\end{array}\right)} \frac{u^{j}}{j} S_{3}=\tan \frac{\theta}{2} \int_{0}^{\theta} \mathrm{d} \phi R_{3 ;-}\left(u_{\phi}\right),
$$

where $R_{3 ;-}(u)$ is defined in Eq. (2.52) and the result can be extracted from Eq. (1.2):

$$
\sum_{j=1}^{\infty} \frac{1}{\left(\begin{array}{c}
2 j \\
j
\end{array}\right)} \frac{u^{j}}{j^{3}}=2\left[\mathrm{Cl}_{3}(\theta)+\theta \mathrm{Cl}_{2}(\theta)-\zeta_{3}\right]+\theta^{2} l_{\theta}
$$

Integrating over $\phi$ we obtain

$$
\sum_{j=1}^{\infty} \frac{1}{\left(\begin{array}{c}
2 j \\
j
\end{array}\right)} \frac{u^{j}}{j} S_{3}=\tan \frac{\theta}{2}\left\{6 \mathrm{Cl}_{4}(\theta)-\theta^{2} \mathrm{Cl}_{2}(\theta)-4 \theta \mathrm{Cl}_{3}(\theta)-2 \theta \zeta_{3}\right\} .
$$

For another sum, $\Sigma_{2,1 ;-; 1}^{1,1 ;-}(u)$, we get

$$
\Sigma_{2,1 ;-; 1}^{1,1 ;-}(u)=\sum_{j=1}^{\infty} \frac{1}{\left(\begin{array}{c}
2 j \\
j
\end{array}\right)} \frac{u^{j}}{j} S_{1} S_{2}=\tan \frac{\theta}{2} \int_{0}^{\theta} \mathrm{d} \phi R_{2,1 ;-}\left(u_{\phi}\right),
$$

where $R_{2,1 ;-}(u)$ is defined in Eq. (2.54). Using Eqs. (2.35), (2.8) and (2.66) to calculate $R_{2,1 ;-}(u)$, and integrating over $\phi$, we get

$$
\begin{gathered}
\sum_{j=1}^{\infty} \frac{1}{\left(\begin{array}{c}
2 j \\
j
\end{array}\right)} \frac{u^{j}}{j} S_{1} S_{2}=\tan \frac{\theta}{2}\left\{\theta^{2} \mathrm{Cl}_{2}(\pi-\theta)-\theta^{2} \mathrm{Cl}_{2}(\theta)-4 \theta \mathrm{Cl}_{3}(\pi-\theta)-4 \theta \mathrm{Cl}_{3}(\theta)\right. \\
\left.-8 \mathrm{Cl}_{4}(\pi-\theta)+6 \mathrm{Cl}_{4}(\theta)+\theta \zeta_{3}-\frac{1}{3} \theta^{3} L_{\theta}\right\} .
\end{gathered}
$$

For the sum $\Sigma_{3 ;-; 1}^{1 ;-}(u)$, this approach is also applicable. In this case we have

$$
\Sigma_{3 ;-; 1}^{1 ;-}(u)=\sum_{j=1}^{\infty} \frac{1}{\left(\begin{array}{c}
2 j \\
j
\end{array}\right)} \frac{u^{j}}{j} S_{1}^{3}=\tan \frac{\theta}{2} \int_{0}^{\theta} \mathrm{d} \phi R_{1,1,1 ;-}\left(u_{\phi}\right),
$$


with $R_{1,1,1 ;-}(u)$ defined in Eq. (2.57). Using Eqs. (2.21), (2.35) and (2.66) to calculate $R_{1,1,1 ;-}(u)$, and integrating over $\phi$, we get

$$
\begin{aligned}
\sum_{j=1}^{\infty} \frac{1}{\left(\begin{array}{c}
2 j \\
j
\end{array}\right)} \frac{u^{j}}{j} S_{1}^{3}= & \tan \frac{\theta}{2}\left\{6 \mathrm{Cl}_{4}(\theta)-24 \mathrm{Cl}_{4}(\pi-\theta)-12 \theta \mathrm{Cl}_{3}(\pi-\theta)-4 \theta \mathrm{Cl}_{3}(\theta)\right. \\
& -24 L_{\theta}\left[\mathrm{Ls}_{3}(\pi-\theta)-\mathrm{Ls}_{3}(\pi)\right]+8\left[\mathrm{Ls}_{4}(\pi-\theta)-\mathrm{Ls}_{4}(\pi)\right]-\theta^{2} \mathrm{Cl}_{2}(\theta) \\
& \left.+3 \theta^{2} \mathrm{Cl}_{2}(\pi-\theta)+24 \mathrm{Cl}_{2}(\pi-\theta) L_{\theta}^{2}-\theta^{3} L_{\theta}-8 \theta L_{\theta}^{3}+7 \zeta_{3} \theta\right\}
\end{aligned}
$$

Combining Eqs. (2.67), (2.68) and (2.69), we successfully reproduce Eq (2.24).

As a further example, let us consider the sum $\Sigma_{2 ; 1 ; 1}^{1 ; 1}(u)$. We obtain

$$
\Sigma_{2 ; 1 ; 1}^{1 ; 1}(u)=\sum_{j=1}^{\infty} \frac{1}{\left(\begin{array}{c}
2 j \\
j
\end{array}\right)} \frac{u^{j}}{j} S_{2} \bar{S}_{1}=\tan \frac{\theta}{2} \int_{0}^{\theta} \mathrm{d} \phi R_{2 ; 1}\left(u_{\phi}\right)
$$

where $R_{2 ; 1}(u)$ corresponds to Eq. (2.55), with some of the contributing sums having $(2 j+1)$ in the denominator. Let us denote them as

$$
\widetilde{\Sigma}_{A ; B ; c ; d}(u) \equiv \sum_{j=1}^{\infty} \frac{u^{j}}{(2 j+1)^{d}} \eta_{A ; B ; c}(j) .
$$

To calculate such sums, it is convenient to apply the differential operator that lowers the value of $d$,

$$
2 u^{1 / 2} \frac{\mathrm{d}}{\mathrm{d} u}\left[u^{1 / 2} \widetilde{\Sigma}_{A ; B ; c ; d}(u)\right] \Leftrightarrow \frac{2}{\cos \frac{\theta}{2}} \frac{\mathrm{d}}{\mathrm{d} \theta}\left[\sin \frac{\theta}{2} \widetilde{\Sigma}_{A ; B ; c ; d}\left(u_{\theta}\right)\right]=\widetilde{\Sigma}_{A ; B ; c ; d-1}\left(u_{\theta}\right) .
$$

In particular, for $d=1$ the sum on the r.h.s. is the standard sum (1.1) which is supposed to be known. Then the result for $\widetilde{\Sigma}_{A ; B ; c ; 1}(u)$ can be obtained by integration,

$$
\widetilde{\Sigma}_{A ; B ; c ; 1}\left(u_{\theta}\right)=\frac{1}{2 \sin \frac{\theta}{2}} \int_{0}^{\theta} \mathrm{d} \phi \cos \frac{\phi}{2} \Sigma_{A ; B ; c}\left(u_{\phi}\right) .
$$

Using the following decomposition

$$
\frac{1}{j^{a}(2 j+1)^{b}}=\sum_{i=0}^{a-1}(-2)^{i}\left(\begin{array}{c}
b-1+i \\
b-1
\end{array}\right) \frac{1}{j^{a-i}}+\sum_{i=0}^{b-1}(-2)^{a}\left(\begin{array}{c}
a-1+i \\
a-1
\end{array}\right) \frac{1}{(2 j+1)^{b-i}},
$$

we are able to rewrite $\widetilde{\Sigma}_{A ; B ; c ; d}(u)$ as a linear combination of the sums, with one of the last two indices equal to zero:

$$
\widetilde{\Sigma}_{A ; B ; c ; d}(u)=\sum_{i=0}^{c-1}(-2)^{i}\left(\begin{array}{c}
d-1+i \\
d-1
\end{array}\right) \widetilde{\Sigma}_{A ; B ; c-i ; 0}(u)+\sum_{i=0}^{d-1}(-2)^{c}\left(\begin{array}{c}
c-1+i \\
c-1
\end{array}\right) \widetilde{\Sigma}_{A ; B ; 0 ; d-i}(u)
$$


For the calculation of the $\widetilde{\Sigma}_{A ; B ; 0 ; d}(u)($ with $c=0)$ we will apply d-times the procedure (2.70)

$$
\left(\frac{2}{\cos \frac{\theta}{2}} \frac{\mathrm{d}}{\mathrm{d} \theta}\right)^{d}\left[\sin \frac{\theta}{2} \widetilde{\Sigma}_{A ; B ; 0 ; d}\left(u_{\theta}\right)\right]=\left(\tan \frac{\theta}{2} \frac{\mathrm{d}}{\mathrm{d} \theta}\right) \Sigma_{A ; B ; 1 ; 0}\left(u_{\theta}\right)
$$

This equation can be easily integrated for some particular cases

$$
\begin{aligned}
& \sum_{j=1}^{\infty} \frac{1}{\left(\begin{array}{c}
2 j \\
j
\end{array}\right)} \frac{u^{j}}{2 j+1}= \frac{\theta}{\sin \theta}-1=\frac{2}{\sin \theta} \mathrm{Ti}_{1}\left(\tan \frac{\theta}{2}\right)-1 \\
& \sum_{j=1}^{\infty} \frac{1}{\left(\begin{array}{c}
2 j \\
j
\end{array}\right)} \frac{u^{j}}{(2 j+1)^{2}}= \frac{2}{\sin \frac{\theta}{2}} \mathrm{Ti}_{2}\left(\tan \frac{\theta}{4}\right)-1 \\
& \sum_{j=1}^{\infty} \frac{1}{\left(\begin{array}{c}
2 j \\
j
\end{array}\right)} \frac{u^{j}}{(2 j+1)} S_{1}= \theta \cot \frac{\theta}{2}+\frac{2}{\sin \theta}\left[\mathrm{Ls}_{2}(\pi-\theta)-\theta L_{\theta}\right]-2 \\
& \sum_{j=1}^{\infty} \frac{1}{\left(\begin{array}{c}
2 j \\
j
\end{array}\right)} \frac{u^{j}}{(2 j+1)} S_{2}= \frac{\theta^{3}}{6 \sin \theta}-2 \theta \cot \frac{\theta}{2}-\frac{1}{2} \theta^{2}+4 \\
& \sum_{j=1}^{\infty} \frac{1}{\left(\begin{array}{c}
2 j \\
j
\end{array}\right)} \frac{u^{j}}{(2 j+1)} \bar{S}_{1}= \frac{1}{\sin \theta}\left[2 \mathrm{Ls}_{2}(\pi-\theta)-2 \theta L_{\theta}+\mathrm{Ls}_{2}(\theta)+\theta l_{\theta}\right] \\
&+\frac{1}{2} \theta \cot \frac{\theta}{2}-\frac{2}{\sin \frac{\theta}{2}} \mathrm{Ti}_{2}\left(\tan \frac{\theta}{4}\right)-1 \\
& \sum_{j=1}^{\infty} \frac{1}{\left(\begin{array}{c}
2 j \\
j
\end{array}\right)} \frac{u^{j}}{(2 j+1)} S_{1}^{2}=-\frac{1}{2} \theta^{2}+4 \cot \frac{\theta}{2}\left[\mathrm{Ls}_{2}(\pi-\theta)-\theta L_{\theta}\right]-4+2 \theta \cot \frac{\theta}{2}+\frac{\theta^{3}}{6 \sin \theta} \\
& \sum_{j=1}^{\infty} \frac{1}{\left(\begin{array}{c}
2 j \\
j
\end{array}\right)} \frac{4}{(2 j+1)} S_{1} S_{2}= 8-\theta^{2} l_{\theta}+2 \theta \mathrm{Cl}_{2}(\pi-\theta)-2 \theta \mathrm{Cl}_{2}(\theta)-4 \mathrm{Cl}_{3}(\pi-\theta)-2 \mathrm{Cl}_{3}(\theta)-\zeta_{3} \\
&+\cot \frac{\theta}{2}\left[\frac{1}{6} \theta^{3}-4 \theta+4 \theta L_{\theta}-4 \mathrm{Cl}_{2}(\pi-\theta)\right] \\
&+\frac{1}{\sin \theta}\left\{\theta^{2}\left[\mathrm{Cl}_{2}(\pi-\theta)-\mathrm{Cl}_{2}(\theta)\right]-4 \theta\left[\mathrm{Cl}_{3}(\pi-\theta)+\mathrm{Cl}_{3}(\theta)\right]\right. \\
&\left.u^{j}(\pi-\theta)-\mathrm{Ls}_{3}(\pi)+\theta L_{\theta}^{2}-2 \mathrm{Ls}_{2}(\pi-\theta) L_{\theta}\right]
\end{aligned}
$$

where $\operatorname{Ti}_{N}(z)$ is the inverse tangent integral (2.16). Together with Eqs. (2.8) and (2.36), this provides us the result for $R_{2 ; 1}(u)$,

$$
R_{2 ; 1}\left(u_{\theta}\right)=4 \mathrm{Cl}_{3}(\pi-\theta)-\mathrm{Cl}_{3}(\theta)-2 \theta \mathrm{Cl}_{2}(\pi-\theta)+4 \zeta_{3}+\frac{1}{2} \theta^{2} l_{\theta}+\frac{1}{6} \theta^{3} \tan \frac{\theta}{2}+\frac{1}{12} \theta^{3} \cot \frac{\theta}{2} .
$$

Finally, using Eq. (2.62) and integrating over $\phi$, we arrive at

$$
\begin{aligned}
\sum_{j=1}^{\infty} \frac{1}{\left(\begin{array}{c}
2 j \\
j
\end{array}\right)} \frac{u^{j}}{j} S_{2} \bar{S}_{1}= & \tan \frac{\theta}{2}\left\{\theta^{2} \mathrm{Cl}_{2}(\pi-\theta)-4 \theta \mathrm{Cl}_{3}(\pi-\theta)-8 \mathrm{Cl}_{4}(\pi-\theta)-\mathrm{Cl}_{4}(\theta)\right. \\
& \left.+\frac{1}{6} \theta^{3} l_{\theta}-\frac{1}{3} \theta^{3} L_{\theta}+4 \theta \zeta_{3}\right\}
\end{aligned}
$$


Using the results for the sums with $c=1$ and applying Eq. (2.32), we obtain the following results for the case $c=2$ :

$$
\begin{aligned}
\sum_{j=1}^{\infty} \frac{1}{\left(\begin{array}{c}
2 j \\
j
\end{array}\right)} \frac{u^{j}}{j^{2}} S_{3}= & \theta^{2} \mathrm{Cl}_{3}(\theta)-6 \theta \mathrm{Cl}_{4}(\theta)-12 \mathrm{Cl}_{5}(\theta)-\theta^{2} \zeta_{3}+12 \zeta_{5} \\
\sum_{j=1}^{\infty} \frac{1}{\left(\begin{array}{c}
2 j \\
j
\end{array}\right)} \frac{u^{j}}{j^{2}} S_{1} S_{2}= & -\frac{1}{3} \theta^{3} \mathrm{Cl}_{2}(\pi-\theta)+2 \theta^{2} \mathrm{Cl}_{3}(\pi-\theta)+\theta^{2} \mathrm{Cl}_{3}(\theta)+8 \theta \mathrm{Cl}_{4}(\pi-\theta) \\
& -6 \theta \mathrm{Cl}_{4}(\theta)-16 \mathrm{Cl}_{5}(\pi-\theta)-12 \mathrm{Cl}_{5}(\theta)+\frac{1}{2} \theta^{2} \zeta_{3}-3 \zeta_{5}, \\
\sum_{j=1}^{\infty} \frac{1}{\left(\begin{array}{c}
2 j \\
j
\end{array}\right)} \frac{u^{j}}{j^{2}} S_{2} \bar{S}_{1}= & -\frac{1}{3} \theta^{3} \mathrm{Cl}_{2}(\pi-\theta)-\frac{1}{6} \theta^{3} \mathrm{Cl}_{2}(\theta)+2 \theta^{2} \mathrm{Cl}_{3}(\pi-\theta)+2 \theta^{2} \zeta_{3}-\frac{1}{2} \theta^{2} \mathrm{Cl}_{3}(\theta) \\
& +8 \theta \mathrm{Cl}_{4}(\pi-\theta)+\theta \mathrm{Cl}_{4}(\theta)-16 \mathrm{Cl}_{5}(\pi-\theta)+2 \mathrm{Cl}_{5}(\theta)-17 \zeta_{5}
\end{aligned}
$$

We note that the results for other sums obtained in the previous sections can be also reproduced by this method. Moreover, it is possible to consider not only the combinations $\left(\bar{S}_{2}+\bar{S}_{1}^{2}\right)$ that correspond to the $J=1$ case of Eq. (2.30) (given in Eq. (2.31)), but also the sums containing $\bar{S}_{2}$ or $\bar{S}_{1}^{2}$ separately (which appear for $J \neq 1$ ). As an example, let us consider the sum

$$
\Sigma_{-; 2 ; 1}^{-; 1}(u)=\sum_{j=1}^{\infty} \frac{1}{\left(\begin{array}{c}
2 j \\
j
\end{array}\right)} \frac{u^{j}}{j} \bar{S}_{2}=\tan \frac{\theta}{2}\left[\theta+\int_{0}^{\theta} \mathrm{d} \phi R_{-; 2}\left(u_{\phi}\right)\right],
$$

where $R_{-; 2}(u)$ corresponds to the case (2.53).

Substituting

$$
R_{-; 2}\left(u_{\theta}\right)=\frac{2}{\sin \frac{\theta}{2}} \mathrm{Ti}_{2}\left(\tan \frac{\theta}{4}\right)+\frac{1}{8} \theta^{2}-1
$$

into Eq. (2.84) and integrating over $\phi$, we arrive at

$$
\sum_{j=1}^{\infty} \frac{1}{\left(\begin{array}{c}
2 j \\
j
\end{array}\right)} \frac{u^{j}}{j} \bar{S}_{2}=\tan \frac{\theta}{2}\left\{4 \operatorname{Ti}_{3}\left(\tan \frac{\theta}{4}\right)+\frac{1}{24} \theta^{3}\right\} .
$$

Moreover, upon applying Eq. (2.32) we can perform another integration and obtain the result for $c=2$,

$$
\sum_{j=1}^{\infty} \frac{1}{\left(\begin{array}{c}
2 j \\
j
\end{array}\right)} \frac{u^{j}}{j^{2}} \bar{S}_{2}=4 \theta \operatorname{Ti}_{3}\left(\tan \frac{\theta}{4}\right)-8\left[\operatorname{Ti}_{2}\left(\tan \frac{\theta}{4}\right)\right]^{2}+\frac{1}{96} \theta^{4} .
$$

Using the connections between $\mathrm{Ti}_{2}\left(\tan \frac{\theta}{4}\right), \mathrm{Ti}_{3}\left(\tan \frac{\theta}{4}\right)$ and the log-sine functions (see Eq. (17) on p. 292 and Eq. (44) on p. 298 of Ref. [2]), we can get other representations,

$$
\begin{aligned}
\sum_{j=1}^{\infty} \frac{1}{\left(\begin{array}{c}
2 j \\
j
\end{array}\right)} \frac{u^{j}}{j} \bar{S}_{2}= & \tan \frac{\theta}{2}\left\{\frac{1}{24} \theta^{3}+\frac{1}{2} \theta \ln ^{2}\left(\tan \frac{\theta}{4}\right)+2 \ln \left(\tan \frac{\theta}{4}\right)\left[\operatorname{Ls}_{2}\left(\frac{\theta}{2}\right)+\operatorname{Ls}_{2}\left(\pi-\frac{\theta}{2}\right)\right]\right. \\
& \left.+2\left[\operatorname{Ls}_{3}\left(\pi-\frac{\theta}{2}\right)-\operatorname{Ls}_{3}(\pi)\right]-2 \operatorname{Ls}_{3}\left(\frac{\theta}{2}\right)+\frac{1}{2} \operatorname{Ls}_{3}(\theta)\right\}
\end{aligned}
$$




$$
\begin{aligned}
\sum_{j=1}^{\infty} \frac{1}{\left(\begin{array}{c}
2 j \\
j
\end{array}\right)} \frac{u^{j}}{j^{2}} \bar{S}_{2}= & 2 \theta\left[\operatorname{Ls}_{3}\left(\pi-\frac{\theta}{2}\right)-\operatorname{Ls}_{3}\left(\frac{\theta}{2}\right)\right]+\frac{1}{2} \theta \operatorname{Ls}_{3}(\theta)-2\left[\operatorname{Ls}_{2}\left(\pi-\frac{\theta}{2}\right)+\operatorname{Ls}_{2}\left(\frac{\theta}{2}\right)\right]^{2} \\
& +\frac{1}{6} \pi^{3} \theta+\frac{1}{96} \theta^{4}
\end{aligned}
$$

Note the appearance of log-sine functions of $\operatorname{arguments} \frac{\theta}{2}$ and $\left(\pi-\frac{\theta}{2}\right)$.

\section{Analytical continuation}

To obtain results valid in other regions of variable $u$ (for $u<0$ and $u>4$ ), we will construct the proper analytical continuation of the expressions presented in the previous section. For generalized log-sine integrals it is described in [16]. Let us introduce a new variable

$$
y \equiv e^{\mathrm{i} \sigma \theta}, \quad \ln (-y-\mathrm{i} \sigma 0)=\ln y-\mathrm{i} \sigma \pi,
$$

where the choice of the sign $\sigma= \pm 1$ is related to the causal " $+\mathrm{i} 0$ " prescription for the propagators. For completeness, we also present the inverse relations,

$$
u=-\frac{(1-y)^{2}}{y}, \quad y=\frac{1-\sqrt{\frac{u}{u-4}}}{1+\sqrt{\frac{u}{u-4}}}, \quad u \frac{\mathrm{d}}{\mathrm{d} u}=-\frac{1-y}{1+y} y \frac{\mathrm{d}}{\mathrm{d} y},
$$

and also expressions for $(1 \pm y)$ in terms of $\theta$,

$$
1-y=2 \sin \frac{\theta}{2} e^{-\mathrm{i} \sigma(\pi-\theta) / 2}, \quad 1+y=2 \cos \frac{\theta}{2} e^{\mathrm{i} \sigma \theta / 2} .
$$

In terms of this variable $y$, the analytic continuation of all generalized log-sine integrals can be expressed in terms of Nielsen polylogarithms, whereas for the function $\Phi(\theta)$ we get

$$
\begin{aligned}
\Phi(\theta)= & \zeta_{3} \ln 2+\frac{1}{2} \zeta_{4}-\mathrm{H}_{-1,0,0,1}(1)+\frac{1}{2}\left[\mathrm{H}_{-1,0,0,1}(y)+\mathrm{H}_{-1,0,0,1}\left(y^{-1}\right)\right] \\
& -\frac{1}{4}\left[\operatorname{Li}_{4}(y)+\operatorname{Li}_{4}\left(y^{-1}\right)\right]-\frac{1}{2}\left[\ln (1+y)-\frac{1}{2} \ln y\right]\left[\operatorname{Li}_{3}(y)+\operatorname{Li}_{3}\left(y^{-1}\right)\right],
\end{aligned}
$$

with $\mathrm{H}_{-1,0,0,1}(y)$ defined in Eq. (2.46).

For the cases involving the inverse tangent integrals $\operatorname{Ti}_{N}\left(\tan \frac{\theta}{2}\right)$ and $\operatorname{Ti}_{N}\left(\tan \frac{\theta}{4}\right)$, the analytic continuation is straightforward (see Eq. (2.16) ),

$$
\begin{array}{ll}
\operatorname{Ti}_{N}\left(\tan \frac{\theta}{2}\right)=-\frac{\sigma}{2 \mathrm{i}}\left[\operatorname{Li}_{N}(\omega)-\operatorname{Li}_{N}(-\omega)\right], & \omega=\frac{1-y}{1+y}=-\mathrm{i} \sigma \tan \frac{\theta}{2} . \\
\operatorname{Ti}_{N}\left(\tan \frac{\theta}{4}\right)=-\frac{\sigma}{2 \mathrm{i}}\left[\operatorname{Li}_{N}\left(\omega_{s}\right)-\operatorname{Li}_{N}\left(-\omega_{s}\right)\right], & \omega_{s}=\frac{1-\sqrt{y}}{1+\sqrt{y}}=-\mathrm{i} \sigma \tan \frac{\theta}{4} .
\end{array}
$$

Below we list the most complicated results, corresponding to the analytical continuation of the results obtained in Section 2:

$$
\sum_{j=1}^{\infty} \frac{1}{\left(\begin{array}{c}
2 j \\
j
\end{array}\right)} \frac{u^{j}}{j^{2}} S_{1}^{2}=-8 \mathrm{~S}_{1,2}(-y) \ln y+4 \mathrm{Li}_{3}(-y) \ln y-2 \mathrm{Li}_{2}(-y) \ln ^{2} y+4\left[\operatorname{Li}_{2}(-y)\right]^{2}
$$




$$
-\frac{1}{24} \ln ^{4} y+4 \zeta_{2} \operatorname{Li}_{2}(-y)+\zeta_{2} \ln ^{2} y+4 \zeta_{3} \ln y+\frac{5}{2} \zeta_{4},
$$

$\sum_{j=1}^{\infty} \frac{1}{\left(\begin{array}{c}2 j \\ j\end{array}\right)} \frac{u^{j}}{j^{2}} S_{1} \bar{S}_{1}=-10 \mathrm{~S}_{1,2}(-y) \ln y+\mathrm{S}_{1,2}\left(y^{2}\right) \ln y-2 \mathrm{~S}_{1,2}(y) \ln y+4\left[\operatorname{Li}_{2}(-y)\right]^{2}$

$-2 \mathrm{Li}_{2}(y) \mathrm{Li}_{2}(-y)+3 \mathrm{Li}_{3}(-y) \ln y-\mathrm{Li}_{3}(y) \ln y-\frac{3}{2} \mathrm{Li}_{2}(-y) \ln ^{2} y+\frac{1}{2} \mathrm{Li}_{2}(y) \ln ^{2} y$

$-\frac{1}{48} \ln ^{4} y+6 \zeta_{2} \operatorname{Li}_{2}(-y)-\zeta_{2} \operatorname{Li}_{2}(y)+\frac{5}{4} \zeta_{2} \ln ^{2} y+\frac{11}{2} \zeta_{3} \ln y+5 \zeta_{4}$,

$\sum_{j=1}^{\infty} \frac{1}{\left(\begin{array}{c}2 j \\ j\end{array}\right)} \frac{u^{j}}{j^{2}}\left(\bar{S}_{2}+\bar{S}_{1}^{2}\right)=-12 \mathrm{~S}_{1,2}(-y) \ln y+2 \mathrm{~S}_{1,2}\left(y^{2}\right) \ln y-6 \mathrm{~S}_{1,2}(y) \ln y+4\left[\operatorname{Li}_{2}(-y)\right]^{2}$

$+\left[\operatorname{Li}_{2}(y)\right]^{2}-4 \mathrm{Li}_{2}(-y) \mathrm{Li}_{2}(y)+2 \mathrm{Li}_{3}(-y) \ln y-\mathrm{Li}_{3}(y) \ln y-\mathrm{Li}_{2}(-y) \ln ^{2} y$

$+\frac{1}{2} \operatorname{Li}_{2}(y) \ln ^{2} y+8 \zeta_{2} \operatorname{Li}_{2}(-y)-4 \zeta_{2} \operatorname{Li}_{2}(y)+\zeta_{2} \ln ^{2} y+8 \zeta_{3} \ln y+10 \zeta_{4}$,

$\sum_{j=1}^{\infty} \frac{1}{\left(\begin{array}{c}2 j \\ j\end{array}\right)} \frac{u^{j}}{j} \mathcal{C}_{1}=\frac{1-y}{1+y}\left[3 \mathrm{Li}_{4}(-y)-3 \mathrm{Li}_{4}(y)+28 \mathrm{~S}_{1,3}(-y)-2 \mathrm{~S}_{1,3}\left(y^{2}\right)+4 \mathrm{~S}_{1,3}(y)\right.$

$-14 \mathrm{~S}_{2,2}(-y)+\mathrm{S}_{2,2}\left(y^{2}\right)-2 \mathrm{~S}_{2,2}(y)+28 \mathrm{~S}_{1,2}(-y) \ln (1+y)-2 \mathrm{~S}_{1,2}\left(y^{2}\right) \ln (1-y)$

$-2 \mathrm{~S}_{1,2}\left(y^{2}\right) \ln (1+y)+4 \mathrm{~S}_{1,2}(y) \ln (1-y)+4 \mathrm{Li}_{3}(-y) \ln (1-y)+2 \mathrm{Li}_{3}(y) \ln (1-y)$

$-10 \mathrm{Li}_{3}(-y) \ln (1+y)+4 \mathrm{Li}_{3}(y) \ln (1+y)-2 \mathrm{Li}_{2}(-y) \ln ^{2}(1-y)$

$-2 \mathrm{Li}_{2}\left(y^{2}\right) \ln (1+y) \ln (1-y)+12 \mathrm{Li}_{2}(-y) \ln ^{2}(1+y)-2 \mathrm{Li}_{2}(y) \ln ^{2}(1+y)$

$-2 \ln y \ln ^{2}(1-y) \ln (1+y)-2 \ln y \ln (1-y) \ln ^{2}(1+y)+4 \ln y \ln ^{3}(1+y)$

$+\frac{1}{2} \ln ^{2} y \ln ^{2}(1-y)+2 \ln ^{2} y \ln (1-y) \ln (1+y)-\frac{5}{2} \ln ^{2} y \ln ^{2}(1+y)-\frac{1}{2} \ln ^{3} y \ln (1-y)$

$+\frac{1}{2} \ln ^{3} y \ln (1+y)+\frac{45}{8} \zeta_{4}-\zeta_{3} \ln (1-y)-13 \zeta_{3} \ln (1+y)+7 \zeta_{3} \ln y+\frac{9}{4} \zeta_{2} \ln ^{2} y$

$\left.-\zeta_{2} \ln ^{2}(1-y)+2 \zeta_{2} \ln (1-y) \ln (1+y)+8 \zeta_{2} \ln ^{2}(1+y)-9 \zeta_{2} \ln y \ln (1+y)\right]$,

$\sum_{j=1}^{\infty} \frac{1}{\left(\begin{array}{c}2 j \\ j\end{array}\right)} \frac{u^{j}}{j} \mathcal{C}_{2}=\frac{1-y}{1+y}\left[2 \mathrm{~S}_{1,3}\left(y^{2}\right)-24 \mathrm{~S}_{1,3}(-y)-6 \mathrm{~S}_{1,3}(y)+12 \mathrm{~S}_{2,2}(-y)-\mathrm{S}_{2,2}\left(y^{2}\right)\right.$

$+3 \mathrm{~S}_{2,2}(y)-2 \mathrm{Li}_{4}(-y)+\frac{5}{2} \operatorname{Li}_{4}(y)-24 \mathrm{~S}_{1,2}(-y) \ln (1+y)-6 \mathrm{~S}_{1,2}(y) \ln (1-y)$

$-\mathrm{Li}_{3}(y) \ln (1-y)+2 \mathrm{~S}_{1,2}\left(y^{2}\right) \ln (1-y)+2 \mathrm{~S}_{1,2}\left(y^{2}\right) \ln (1+y)-4 \operatorname{Li}_{3}(-y) \ln (1-y)$

$+8 \mathrm{Li}_{3}(-y) \ln (1+y)-4 \mathrm{Li}_{3}(y) \ln (1+y)+2 \operatorname{Li}_{2}(-y) \ln ^{2}(1-y)-\operatorname{Li}_{2}(y) \ln ^{2}(1-y)$

$+4 \mathrm{Li}_{2}(-y) \ln (1-y) \ln (1+y)+4 \mathrm{Li}_{2}(y) \ln (1-y) \ln (1+y)-10 \mathrm{Li}_{2}(-y) \ln ^{2}(1+y)$

$+2 \mathrm{Li}_{2}(y) \ln ^{2}(1+y)-\frac{1}{3} \ln y \ln ^{3}(1-y)+2 \ln y \ln ^{2}(1-y) \ln (1+y)$

$+2 \ln y \ln (1-y) \ln ^{2}(1+y)-\frac{10}{3} \ln y \ln ^{3}(1+y)-\frac{1}{4} \ln ^{2} y \ln ^{2}(1-y)$

$-2 \ln ^{2} y \ln (1-y) \ln (1+y)+2 \ln ^{2} y \ln ^{2}(1+y)+\frac{5}{12} \ln ^{3} y \ln (1-y)-\frac{1}{3} \ln ^{3} y \ln (1+y)$

$-\frac{1}{96} \ln ^{4} y-\frac{9}{4} \zeta_{4}+2 \zeta_{3} \ln (1-y)+11 \zeta_{3} \ln (1+y)-\frac{13}{2} \zeta_{3} \ln y-7 \zeta_{2} \ln ^{2}(1+y)$

$+2 \zeta_{2} \ln ^{2}(1-y)-2 \zeta_{2} \ln (1-y) \ln (1+y)-\frac{7}{4} \zeta_{2} \ln ^{2} y-\zeta_{2} \ln y \ln (1-y)$

$\left.+8 \zeta_{2} \ln y \ln (1+y)\right]$,

$\sum_{j=1}^{\infty} \frac{1}{\left(\begin{array}{c}2 j \\ j\end{array}\right)} \frac{u^{j}}{j^{3}} S_{1}=4 H_{-1,0,0,1}(-y)+\mathrm{S}_{2,2}\left(y^{2}\right)-4 \mathrm{~S}_{2,2}(y)-4 \mathrm{~S}_{2,2}(-y)-6 \operatorname{Li}_{4}(-y)$ 


$$
\begin{aligned}
& -2 \mathrm{Li}_{4}(y)+4 \mathrm{~S}_{1,2}(-y) \ln y+4 \mathrm{~S}_{1,2}(y) \ln y-2 \mathrm{~S}_{1,2}\left(y^{2}\right) \ln (y)+4 \operatorname{Li}_{3}(-y) \ln (1-y) \\
& +2 \mathrm{Li}_{3}(-y) \ln y+2 \mathrm{Li}_{3}(y) \ln y-\mathrm{Li}_{2}(y) \ln ^{2} y-4 \mathrm{Li}_{2}(-y) \ln y \ln (1-y) \\
& -\frac{1}{3} \ln ^{3} y \ln (1-y)+\frac{1}{24} \ln ^{4} y+2 \zeta_{2} \operatorname{Li}_{2}(y)-\frac{1}{2} \zeta_{2} \ln ^{2} y+2 \zeta_{2} \ln y \ln (1-y) \\
& +6 \zeta_{3} \ln (1-y)-3 \zeta_{3} \ln y-4 \zeta_{4} \text {, } \\
& \sum_{j=1}^{\infty} \frac{1}{\left(\begin{array}{c}
2 j \\
j
\end{array}\right)} \frac{u^{j}}{j^{3}} \bar{S}_{1}=4 H_{-1,0,0,1}(-y)+\mathrm{S}_{2,2}\left(y^{2}\right)-8 \mathrm{~S}_{2,2}(y)-4 \mathrm{~S}_{2,2}(-y)-6 \operatorname{Li}_{4}(-y) \\
& +2 \mathrm{Li}_{4}(y)-\left[\operatorname{Li}_{2}(y)\right]^{2}+4 \mathrm{~S}_{1,2}(-y) \ln y+8 \mathrm{~S}_{1,2}(y) \ln y-2 \mathrm{~S}_{1,2}\left(y^{2}\right) \ln y+\frac{1}{48} \ln ^{4} y \\
& +4 \mathrm{Li}_{3}(-y) \ln (1-y)-4 \mathrm{Li}_{3}(y) \ln (1-y)+2 \mathrm{Li}_{3}(-y) \ln y-4 \mathrm{Li}_{2}(-y) \ln y \ln (1-y) \\
& +2 \mathrm{Li}_{2}(y) \ln y \ln (1-y)-\frac{1}{2} \mathrm{Li}_{2}(y) \ln ^{2} y-\frac{1}{6} \ln ^{3} y \ln (1-y)+4 \zeta_{2} \ln y \ln (1-y) \\
& -\zeta_{2} \ln ^{2} y+10 \zeta_{3} \ln (1-y)-5 \zeta_{3} \ln y+4 \zeta_{2} \operatorname{Li}_{2}(y)-\frac{19}{2} \zeta_{4} \text {, } \\
& \sum_{j=1}^{\infty} \frac{1}{\left(\begin{array}{c}
2 j \\
j
\end{array}\right)} \frac{u^{j}}{j} S_{1}^{3}=\frac{1-y}{1+y}\left[-48 \mathrm{~S}_{1,2}(-y) \ln (1+y)-48 \mathrm{~S}_{1,3}(-y)+24 \mathrm{~S}_{2,2}(-y)\right. \\
& -12 \zeta_{2} \ln ^{2}(1+y)-24 \ln ^{2}(1+y) \operatorname{Li}_{2}(-y)+24 \zeta_{3} \ln (1+y)+24 \ln (1+y) \operatorname{Li}_{3}(-y) \\
& -8 \ln y \ln ^{3}(1+y)+12 \zeta_{2} \ln y \ln (1+y)+6 \ln ^{2} y \ln ^{2}(1+y)-\ln ^{3} y \ln (1+y) \\
& +\frac{1}{24} \ln ^{4} y-\frac{3}{2} \zeta_{2} \ln ^{2} y+3 \ln ^{2} y \operatorname{Li}_{2}(-y)+\ln ^{2} y \operatorname{Li}_{2}(y)-5 \zeta_{3} \ln y-12 \ln y \operatorname{Li}_{3}(-y) \\
& \left.-4 \ln y \mathrm{Li}_{3}(y)+\frac{3}{2} \zeta_{4}+12 \mathrm{Li}_{4}(-y)+6 \mathrm{Li}_{4}(y)\right] \\
& \sum_{j=1}^{\infty} \frac{1}{\left(\begin{array}{c}
2 j \\
j
\end{array}\right)} \frac{u^{j}}{j^{2}} S_{3}=-12 \mathrm{Li}_{5}(y)+6 \ln y \mathrm{Li}_{4}(y)-\ln ^{2} y \mathrm{Li}_{3}(y)-\frac{1}{120} \ln ^{5} y \\
& +\zeta_{3} \ln ^{2} y+6 \zeta_{4} \ln y+12 \zeta_{5} \\
& \sum_{j=1}^{\infty} \frac{1}{\left(\begin{array}{c}
2 j \\
j
\end{array}\right)} \frac{u^{j}}{j^{2}} S_{1} S_{2}=-12 \operatorname{Li}_{5}(y)-16 \operatorname{Li}_{5}(-y)+6 \ln y \operatorname{Li}_{4}(y)+8 \ln y \operatorname{Li}_{4}(-y) \\
& -\ln ^{2} y \operatorname{Li}_{3}(y)-2 \ln ^{2} y \operatorname{Li}_{3}(-y)+\frac{1}{3} \ln ^{3} y \operatorname{Li}_{2}(-y)+\frac{1}{120} \ln ^{5} y-\frac{1}{6} \zeta_{2} \ln ^{3} y \\
& -\frac{1}{2} \zeta_{3} \ln ^{2} y-\zeta_{4} \ln y-3 \zeta_{5} \\
& \sum_{j=1}^{\infty} \frac{1}{\left(\begin{array}{c}
2 j \\
j
\end{array}\right)} \frac{u^{j}}{j^{2}} S_{2} \bar{S}_{1}=2 \operatorname{Li}_{5}(y)-16 \operatorname{Li}_{5}(-y)-\ln y \operatorname{Li}_{4}(y)+8 \ln y \operatorname{Li}_{4}(-y) \\
& +\frac{1}{2} \ln ^{2} y \mathrm{Li}_{3}(y)-2 \ln ^{2} y \mathrm{Li}_{3}(-y)+\frac{1}{3} \ln ^{3} y \mathrm{Li}_{2}(-y)-\frac{1}{6} \ln ^{3} y \mathrm{Li}_{2}(y)+\frac{1}{240} \ln ^{5} y \\
& -\frac{1}{3} \zeta_{2} \ln ^{3} y-2 \zeta_{3} \ln ^{2} y-8 \zeta_{4} \ln y-17 \zeta_{5},
\end{aligned}
$$

where we have used the relations (A.6) and (A.7).

For the results involving $\operatorname{Lsc}_{2,3}(\theta)$ we can use Eq. (2.18) to express them in terms of Ti function, and then employ Eq. (3.5). For example, starting from Eq. (C.5) we obtain

$$
\begin{aligned}
& \sum_{j=1}^{\infty} \frac{1}{\left(\begin{array}{c}
2 j \\
j
\end{array}\right)} \frac{u^{j}}{j} S_{1}^{2} \bar{S}_{1}=\frac{1-y}{1+y}\left\{\operatorname{Li}_{4}(y)+8 \operatorname{Li}_{4}(-y)-4 \operatorname{Li}_{4}(\omega)+4 \operatorname{Li}_{4}(-\omega)\right. \\
& +2 \mathrm{~S}_{1,3}\left(y^{2}\right)-8 \mathrm{~S}_{1,3}(y)-48 \mathrm{~S}_{1,3}(-y)-\mathrm{S}_{2,2}\left(y^{2}\right)+4 \mathrm{~S}_{2,2}(y)+24 \mathrm{~S}_{2,2}(-y) \\
& +2 \ln (1+y)\left[\mathrm{S}_{1,2}\left(y^{2}\right)-24 \mathrm{~S}_{1,2}(-y)+10 \operatorname{Li}_{3}(-y)-2 \operatorname{Li}_{3}(y)\right]
\end{aligned}
$$




$$
\begin{aligned}
& +2 \ln (1-y)\left[\mathrm{S}_{1,2}\left(y^{2}\right)-4 \mathrm{~S}_{1,2}(y)-2 \operatorname{Li}_{3}(-y)\right]-8 \ln y \operatorname{Li}_{3}(-y) \\
& +2 \operatorname{Li}_{2}(-y)\left[\ln ^{2}(1-y)+2 \ln (1-y) \ln (1+y)-11 \ln ^{2}(1+y)+\ln ^{2} y\right] \\
& +2 \operatorname{Li}_{2}(y)\left[\ln ^{2}(1+y)-\ln ^{2}(1-y)+2 \ln (1-y) \ln (1+y)\right]+\frac{1}{48} \ln ^{4} y \\
& +2 \ln y\left[\ln (1+y) \ln ^{2}(1-y)+\ln (1-y) \ln ^{2}(1+y)-\frac{1}{3} \ln ^{3}(1-y)-\frac{11}{3} \ln ^{3}(1+y)\right] \\
& -2 \ln ^{2} y \ln (1-y) \ln (1+y)+5 \ln ^{2} y \ln ^{2}(1+y)+\frac{1}{6} \ln ^{3} y \ln (1-y)-\frac{2}{3} \ln ^{3} y \ln (1+y) \\
& +\zeta_{2}\left[3 \ln ^{2}(1-y)-2 \ln (1-y) \ln (1+y)-13 \ln ^{2}(1+y)-2 \ln y \ln (1-y)+14 \ln y \ln (1+y)-\frac{3}{2} \ln ^{2} y\right] \\
& \left.+\zeta_{3}[3 \ln (1-y)-6 \ln y+23 \ln (1+y)]+\frac{33}{4} \zeta_{4}\right\},
\end{aligned}
$$

where $\omega=(1-y) /(1+y)$, see Eq. (3.5). We note that the analytic continuation of $\operatorname{Lsc}_{2,3}(\theta)$ can also be obtained directly, using the integral representation (2.14). This procedure is described in Appendix A, Eqs. A.26 A.32.

The analytic continuation of the sum involving $\bar{S}_{2}$ can be presented in terms of the variable $\omega_{s}$ given in Eq. (3.6),

$$
\sum_{j=1}^{\infty} \frac{1}{\left(\begin{array}{c}
2 j \\
j
\end{array}\right)} \frac{u^{j}}{j^{2}} \bar{S}_{2}=2 \ln y\left[\operatorname{Li}_{3}\left(\omega_{s}\right)-\operatorname{Li}_{3}\left(-\omega_{s}\right)\right]+2\left[\operatorname{Li}_{2}\left(\omega_{s}\right)-\operatorname{Li}_{2}\left(-\omega_{s}\right)\right]^{2}+\frac{1}{96} \ln ^{4} y .
$$

The results for lower values of $c$ can be deduced using

$$
\sum_{j=1}^{\infty} \frac{u^{j}}{j^{c}} f(j)=u \frac{\mathrm{d}}{\mathrm{d} u} \sum_{j=1}^{\infty} \frac{u^{j}}{j^{c+1}} f(j)
$$

For example,

$$
\begin{aligned}
\sum_{j=1}^{\infty} \frac{1}{\left(\begin{array}{c}
2 j \\
j
\end{array}\right)} \frac{u^{j}}{j^{2}} S_{1}= & 4 \operatorname{Li}_{3}(-y)-2 \operatorname{Li}_{2}(-y) \ln y-\frac{1}{6} \ln ^{3} y+3 \zeta_{3}+\zeta_{2} \ln y \\
\sum_{j=1}^{\infty} \frac{1}{\left(\begin{array}{c}
2 j \\
j
\end{array}\right)} \frac{u^{j}}{j^{2}} \bar{S}_{1}= & -2 \operatorname{Li}_{3}(y)+4 \operatorname{Li}_{3}(-y)-2 \operatorname{Li}_{2}(-y) \ln y+\operatorname{Li}_{2}(y) \ln y \\
& -\frac{1}{12} \ln ^{3} y+2 \zeta_{2} \ln y+5 \zeta_{3} .
\end{aligned}
$$

The results for these two sums can be extracted from Ref. [14]. Further results for the sums are collected in Appendix C.

\section{Application to Feynman diagrams}

Below we present results for the $\varepsilon$-expansion of one- and two-loop master integrals shown in Fig. 1. In the rest of this paper we use the notation $u=p^{2} / m^{2}$. 


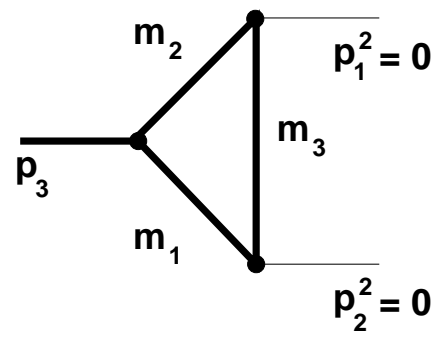

$J_{3}$

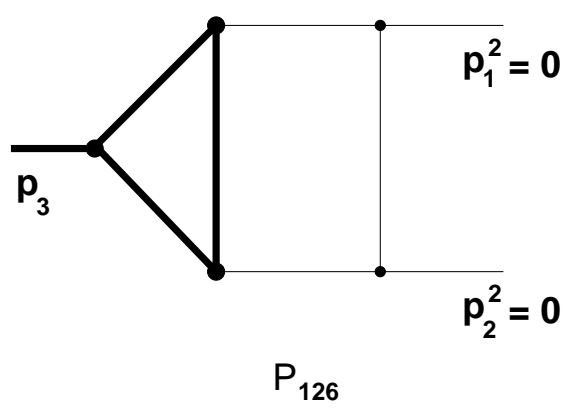

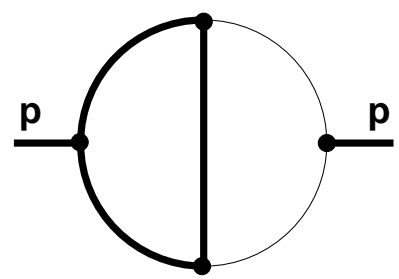

F10101

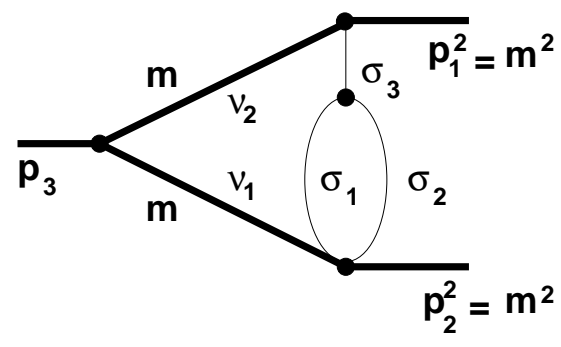

$\mathrm{K}\left(v_{1}, v_{2}, \sigma_{1}, \sigma_{2}, \sigma_{3}\right)$

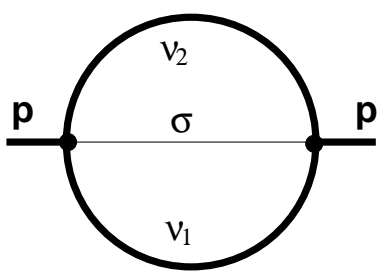

$\operatorname{J} 011\left(\sigma, v_{1}, v_{2}\right)$

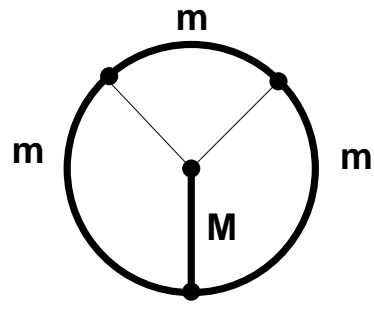

$\mathrm{D}_{4}$

Figure 1: One- two- and three-loop diagrams considered in the paper. Bold and thin lines correspond to massive and massless propagators, respectively.

\subsection{One-loop vertex}

Let us consider a one-loop triangle diagram with $m_{1}=m_{2}=m_{3} \equiv m, p_{1}^{2}=p_{2}^{2}=0$, with an arbitrary (off-shell) value of $p_{3}^{2} \equiv p^{2}$. Such diagrams occur, for example, in Higgs decay into two photons or two gluons via a massive quark loop. Following the notation of Ref. [10], we will denote this integral (with unit powers of propagators) as $J_{3}(1,1,1 ; m)$. According to Eq. (40) of Ref. [10], the result in an arbitrary space-time dimension $n=4-2 \varepsilon$ is

$$
\left.J_{3}(1,1,1 ; m)\right|_{p_{1}^{2}=p_{2}^{2}=0}=-\frac{1}{2} \mathrm{i} \pi^{2-\varepsilon}\left(m^{2}\right)^{-1-\varepsilon} \Gamma(1+\varepsilon){ }_{3} F_{2}\left(\begin{array}{c|c}
1,1,1+\varepsilon & \frac{p^{2}}{2} \\
\frac{3}{2}, 2 & 4 m^{2}
\end{array}\right) .
$$

For this integral a number of one-fold integral representations are available, see Eqs. (3.10)(3.11) in Ref. [16]. Expanding in $\varepsilon$ we get

$$
\begin{gathered}
\left.J_{3}(1,1,1 ; m)\right|_{p_{1}^{2}=p_{2}^{2}=0}=-\mathrm{i} \pi^{2-\varepsilon} \Gamma(1+\varepsilon) \frac{\left(m^{2}\right)^{-\varepsilon}}{p^{2}} \sum_{j=1}^{\infty} \frac{1}{\left(\begin{array}{c}
2 j \\
j
\end{array}\right)} \frac{u^{j}}{j^{2}}\left[1+\varepsilon S_{1}+\frac{1}{2} \varepsilon^{2}\left(S_{1}^{2}-S_{2}\right)\right. \\
\left.+\frac{1}{6} \varepsilon^{3}\left(S_{1}^{3}-3 S_{1} S_{2}+2 S_{3}\right)+\frac{1}{24} \varepsilon^{4}\left(S_{1}^{4}-6 S_{1}^{2} S_{2}+8 S_{1} S_{3}+3 S_{2}^{2}-6 S_{4}\right)+\mathcal{O}\left(\varepsilon^{5}\right)\right] .
\end{gathered}
$$

Substituting results for the occurring inverse binomial sums, we obtain

$$
\left.J_{3}(1,1,1 ; m)\right|_{p_{1}^{2}=p_{2}^{2}=0}=-\mathrm{i} \pi^{2-\varepsilon} \Gamma(1+\varepsilon) \frac{\left(m^{2}\right)^{-\varepsilon}}{p^{2}}\left\{-\frac{1}{2} \ln ^{2} y\right.
$$




$$
\begin{aligned}
& +\varepsilon\left[4 \mathrm{Li}_{3}(-y)-2 \mathrm{Li}_{2}(-y) \ln y-\frac{1}{6} \ln ^{3} y+\zeta_{2} \ln y+3 \zeta_{3}\right] \\
& +\varepsilon^{2}\left[2\left[\mathrm{Li}_{2}(-y)\right]^{2}+2 \mathrm{Li}_{3}(-y) \ln y-4 \mathrm{~S}_{1,2}(-y) \ln y-\mathrm{Li}_{2}(-y) \ln ^{2} y\right. \\
& \left.\left.+2 \zeta_{2} \operatorname{Li}_{2}(-y)+\frac{1}{2} \zeta_{2} \ln ^{2} y+2 \zeta_{3} \ln y-\frac{1}{24} \ln ^{4} y+\frac{5}{4} \zeta_{4}\right]+\mathcal{O}\left(\varepsilon^{3}\right)\right\} \cdot(4.3)
\end{aligned}
$$

These results correspond to the analytic continuation of Eqs. (3.13), (3.14) and (3.16) from Ref. [16].

The $\varepsilon^{3}$ term is Eq. (4.2) contains the same combination of sums as $\mathcal{C}_{0}$ in Eq. (2.5). However, the sum in Eq. (4.2) contains $j^{2}$ in the denominator, i.e., it corresponds to the case $c=2$. Using the result (2.24) (with $c=1$ ) together with (2.32), we obtain a one-fold integral representation for the $\varepsilon^{3}$-term in Eq. (4.2). Analyzing it, we see that we get only one new non-trivial integral,

$$
\int_{0}^{\theta} \mathrm{d} \phi \operatorname{Ls}_{2}(\phi) \ln ^{2}\left|2 \sin \frac{\phi}{2}\right|
$$

while all other terms can be expressed in terms of known functions. For $\theta=\frac{2 \pi}{3}$ the integral (4.4) is connected with the new element $\chi_{5}$ of the odd basis, whereas for $\theta=\frac{\pi}{2}$ it is related to the new element $\widetilde{\chi}_{5}$ of the even basis, see Section 3.3 in Ref. [16] and Eqs. (14)-(15) in Ref. [24].

At the same time, the $\varepsilon^{4}$-term of Eq. (4.2) (and all other even powers of $\varepsilon$ ) can be calculated in terms of log-sine functions, so that their analytic continuation can be expressed in terms of Nielsen polylogarithms. Namely, for the $\varepsilon^{4}$-term, combining Eqs. (3.14), (3.19) from Ref. [16] and Eq. (2.34), we obtain the result for the sum

$$
\sum_{j=1}^{\infty} \frac{1}{\left(\begin{array}{c}
2 j \\
j
\end{array}\right)} \frac{u^{j}}{j^{c}}\left(S_{1}^{4}-6 S_{1}^{2} S_{2}+8 S_{1} S_{3}-3 S_{2}^{2}\right)
$$

with $c=2$. Using Eq. (3.20), we also obtain the result for the case $c=1$.

\subsection{Two-loop self-energy integral $F_{10101}$}

This integral is a good illustration of the application of general expressions given in Section 3. The off-shell result for this integral in arbitrary dimension was presented in [34] (where it was called $\widetilde{I}_{3}$, see Eq. (22) of [34]). For unit powers of propagators, the result reads ${ }^{6}$

$$
\begin{aligned}
& m^{2+4 \varepsilon}(1-2 \varepsilon) F_{10101}\left(p^{2}, m\right)=\frac{1}{\left(1-\varepsilon^{2}\right)(1+2 \varepsilon)}{ }_{4} F_{3}\left(\begin{array}{c}
1,1+\varepsilon, 1+\varepsilon, 1+2 \varepsilon \\
\frac{3}{2}+\varepsilon, 2+\varepsilon, 2-\varepsilon
\end{array} \mid \frac{p^{2}}{4 m^{2}}\right) \\
& -\frac{1}{2 \varepsilon(1+\varepsilon)}{ }_{3} F_{2}\left(\begin{array}{c}
1,1+\varepsilon, 1+\varepsilon \\
\frac{3}{2}, 2+\varepsilon
\end{array} \mid \frac{p^{2}}{4 m^{2}}\right)+\frac{1}{2 \varepsilon} \frac{\Gamma^{2}(1-\varepsilon)}{\Gamma(1-2 \varepsilon)}\left(-\frac{m^{2}}{p^{2}}\right)^{\varepsilon}{ }_{3} F_{2}\left(\begin{array}{c}
1,1,1+\varepsilon \\
\frac{3}{2}, 2
\end{array} \mid \frac{p^{2}}{4 m^{2}}\right) .
\end{aligned}
$$

\footnotetext{
${ }^{6}$ In given normalization each loop is divided by $\pi^{2-\varepsilon} \Gamma(1+\varepsilon)$.
} 
Expanding in $\varepsilon$, we obtain

$$
\begin{aligned}
& p^{2}\left(m^{2}\right)^{2 \varepsilon}(1-2 \varepsilon) F_{10101}\left(p^{2}, m\right) \\
& =\sum_{j=1}^{\infty} \frac{1}{\left(\begin{array}{c}
2 j \\
j
\end{array}\right)} \frac{u^{j}}{j^{2}}\left\{\frac{3}{j}-\ln (-u)+\varepsilon\left[-\frac{1}{j^{2}}+\frac{11}{j} S_{1}-\frac{4}{j} \bar{S}_{1}-\ln (-u) S_{1}+\frac{1}{2} \ln ^{2}(-u)-\zeta_{2}\right]+\mathcal{O}\left(\varepsilon^{2}\right)\right\} \\
& =6 \operatorname{Li}_{3}(y)-6 \operatorname{Li}_{2}(y) \ln y-2 \ln ^{2} y \ln (1-y)-6 \zeta_{3} \\
& +\varepsilon\left\{28 H_{-1,0,0,1}(-y)+7 \mathrm{~S}_{2,2}\left(y^{2}\right)-28 \mathrm{~S}_{2,2}(-y)-16 \mathrm{~S}_{2,2}(y)-42 \operatorname{Li}_{4}(-y)-26 \operatorname{Li}_{4}(y)\right. \\
& +4\left[\operatorname{Li}_{2}(y)\right]^{2}+16 \mathrm{~S}_{1,2}(y) \ln y-14 \mathrm{~S}_{1,2}\left(y^{2}\right) \ln y+28 \mathrm{~S}_{1,2}(-y) \ln y+18 \operatorname{Li}_{3}(-y) \ln y \\
& +20 \operatorname{Li}_{3}(y) \ln y+20 \operatorname{Li}_{3}(-y) \ln (1-y)+12 \operatorname{Li}_{3}(y) \ln (1-y)-2 \operatorname{Li}_{2}(-y) \ln ^{2} y-9 \operatorname{Li}_{2}(y) \ln ^{2} y \\
& -24 \operatorname{Li}_{2}(-y) \ln y \ln (1-y)-4 \operatorname{Li}_{2}(y) \ln y \ln (1-y)-2 \ln ^{3} y \ln (1-y)+6 \zeta_{2} \operatorname{Li}_{2}(y) \\
& \left.+4 \zeta_{2} \ln y \ln (1-y)-12 \zeta_{3} \ln y+24 \zeta_{3} \ln (1-y)-9 \zeta_{4}\right\}+\mathcal{O}\left(\varepsilon^{2}\right) .
\end{aligned}
$$

The result for the finite part coincides with [35], whereas the result for the $\varepsilon$-term is new.

\subsection{Two-loop sunset-type diagram $J_{011}$}

Let us consider sunset-type diagrams with two equal masses and one zero mass (see Fig. 1). The off-shell result for the sunset-type integral $J_{011}$ with arbitrary powers of propagators has been obtained ${ }^{7}$ in Refs. [34,36] by using the Mellin-Barnes technique [10]:

$$
\begin{aligned}
J_{011}\left(\sigma, \nu_{1}, \nu_{2} ; p^{2}, m\right)= & \left(m^{2}\right)^{n-\sigma-\nu_{1}-\nu_{2}} \frac{\Gamma\left(\nu_{1}+\nu_{2}+\sigma-n\right) \Gamma\left(\frac{n}{2}-\sigma\right) \Gamma\left(\nu_{2}+\sigma-\frac{n}{2}\right) \Gamma\left(\nu_{1}+\sigma-\frac{n}{2}\right)}{\Gamma\left(\nu_{1}\right) \Gamma\left(\nu_{2}\right) \Gamma\left(\frac{n}{2}\right) \Gamma\left(\nu_{1}+\nu_{2}+2 \sigma-n\right) \Gamma^{2}\left(3-\frac{n}{2}\right)} \\
& \times{ }_{4} F_{3}\left(\begin{array}{c}
\sigma, \nu_{1}+\nu_{2}+\sigma-n, \nu_{2}+\sigma-\frac{n}{2}, \nu_{1}+\sigma-\frac{n}{2} \\
\frac{n}{2}, \sigma+\frac{1}{2}\left(\nu_{1}+\nu_{2}-n\right), \sigma+\frac{1}{2}\left(\nu_{1}+\nu_{2}+1-n\right)
\end{array}\right) \cdot\left(\frac{p^{2}}{4 m^{2}}\right) \cdot(4.7)
\end{aligned}
$$

For simplicity, in the definition of the integral $J_{011}$ we will omit the arguments $p^{2}$ and $m$

$$
J_{011}\left(\sigma, \nu_{1}, \nu_{2}\right) \equiv J_{011}\left(\sigma, \nu_{1}, \nu_{2} ; p^{2}, m\right)
$$

Let us remind that for the integrals $J_{011}$ with different integer values of $\sigma$ and $\nu_{i}$ there are two master integrals [38] of this type, $J_{011}(1,1,1)$ and $J_{011}(1,1,2)$. However, two other independent combinations of the integrals of this type happen to be more suitable for constructing the $\varepsilon$-expansion, $J_{011}(1,2,2)$ and $\left[J_{011}(1,2,2)+2 J_{011}(2,1,2)\right]$ (see also in Ref. [39]). The latter combination corresponds to the integral $J_{011}(1,1,1)$ in $2-2 \varepsilon$ dimensions [40]. In Ref. [18] $J_{011}(1,1,3)$ has been used as the second integral. To construct the $\varepsilon$-expansion of the integrals $J_{011}(1,1,1)$ and $J_{011}(1,1,2)$ up to order $\varepsilon^{2}$, the integral $J_{011}(1,2,2)$ should also be expanded up to $\varepsilon^{2}$, whereas $\left[J_{011}(1,2,2)+2 J_{011}(2,1,2)\right]$ or $J_{011}(1,1,3)$ up to the order $\varepsilon$ only. In Appendix $\mathrm{C}$ we give an example of a realistic calculation which demonstrates the required orders of the $\varepsilon$-expansion for the integrals involved.

\footnotetext{
${ }^{7}$ The imaginary part of the sunset diagrams in an arbitrary dimension is presented in [37].
} 
For $J_{011}(1,2,2)$ we have obtained

$$
\begin{aligned}
J_{011}( & , 2,2)=\frac{\left(m^{2}\right)^{-1-2 \varepsilon}}{(1-\varepsilon)(1+2 \varepsilon)}{ }_{3} F_{2}\left(\begin{array}{c}
1,1+\varepsilon, 1+2 \varepsilon \\
\frac{3}{2}+\varepsilon, 2-\varepsilon
\end{array} \mid \frac{p^{2}}{4 m^{2}}\right) \\
= & 2 \frac{\left(m^{2}\right)^{-2 \varepsilon}}{p^{2}} \sum_{j=1}^{\infty} \frac{1}{\left(\begin{array}{c}
2 j \\
j
\end{array}\right)} \frac{u^{j}}{j^{2}}\left\{1+\varepsilon\left[\frac{1}{j}+5 S_{1}-2 \bar{S}_{1}\right]\right. \\
& +\varepsilon^{2}\left[\frac{1}{j^{2}}+\frac{5}{j} S_{1}-\frac{2}{j} \bar{S}_{1}+\frac{25}{2} S_{1}^{2}-10 S_{1} \bar{S}_{1}-\frac{5}{2} S_{2}+2 \bar{S}_{1}^{2}+2 \bar{S}_{2}\right] \\
& +\varepsilon^{3}\left[\frac{1}{j^{3}}+\frac{5}{j^{2}} S_{1}-\frac{2}{j^{2}} \bar{S}_{1}+\frac{25}{2 j} S_{1}^{2}-\frac{10}{j} S_{1} \bar{S}_{1}-\frac{5}{2 j} S_{2}+\frac{2}{j}\left(\bar{S}_{1}^{2}+\bar{S}_{2}\right)+\frac{125}{6} S_{1}^{3}-25 S_{1}^{2} \bar{S}_{1}\right. \\
& \left.\left.-\frac{25}{2} S_{1} S_{2}+\frac{11}{3} S_{3}+5 S_{2} \bar{S}_{1}+10 S_{1}\left(\bar{S}_{1}^{2}+\bar{S}_{2}\right)-\frac{4}{3}\left(\bar{S}_{1}^{3}+3 \bar{S}_{1} \bar{S}_{2}+2 \bar{S}_{3}\right)\right]+\mathcal{O}\left(\varepsilon^{4}\right)\right\} \\
= & 2 \frac{\left(m^{2}\right)^{-2 \varepsilon}}{p^{2}}(1-y)^{2 \varepsilon} y^{2 \varepsilon}\left\{-\frac{1}{2} \ln ^{2} y\right. \\
& +\varepsilon\left[\frac{1}{2} \ln ^{3} y+\zeta_{2} \ln y-6 \ln y \operatorname{Li}_{2}(-y)-4 \ln y \operatorname{Li}_{2}(y)+3 \zeta_{3}+12 \operatorname{Li}_{3}(-y)+6 \operatorname{Li}_{3}(y)\right] \\
& +\varepsilon^{2}\left[12 H_{-1,0,0,1}(-y)-12 \ln y \mathrm{~S}_{1,2}(-y)-12 \ln y \mathrm{~S}_{1,2}\left(y^{2}\right)+8 \ln y \mathrm{~S}_{1,2}(y)-12 \mathrm{~S}_{2,2}(-y)\right. \\
& +3 \mathrm{~S}_{2,2}\left(y^{2}\right)-12 \ln (1-y) \operatorname{Li}_{3}(-y)-\frac{7}{24} \ln { }^{4} y-\frac{1}{2} \zeta_{2} \ln ^{2} y+2 \zeta_{3} \ln y+6 \ln y \operatorname{Li}_{3}(-y) \\
& +8 \ln y \operatorname{Li}_{3}(y)+6 \zeta_{2} \operatorname{Li}_{2}(-y)+4 \zeta_{2} \operatorname{Li}_{2}(y)+\frac{13}{4} \zeta_{4}+18\left[\operatorname{Li}_{2}(-y)\right]^{2} \\
& \left.\left.+12 \operatorname{Li}_{2}(y) \operatorname{Li}_{2}(-y)+4\left[\operatorname{Li}_{2}(y)\right]^{2}-\frac{9}{4} \operatorname{Li}_{4}\left(y^{2}\right)\right]+\mathcal{O}\left(\varepsilon^{3}\right)\right\}
\end{aligned}
$$

where the result for the $\varepsilon^{2}$-term is new.

For another combination, we get

$$
\begin{aligned}
& J_{011}(1,2,2)+2 J_{011}(2,1,2)=-\frac{\left(m^{2}\right)^{-1-2 \varepsilon}}{\varepsilon(1+2 \varepsilon)}{ }_{3} F_{2}\left(\begin{array}{c}
1,1+\varepsilon, 1+2 \varepsilon \\
\frac{3}{2}+\varepsilon, 1-\varepsilon
\end{array} \mid \frac{p^{2}}{4 m^{2}}\right) \\
& =-\frac{2\left(m^{2}\right)^{-2 \varepsilon}}{p^{2}} \sum_{j=1}^{\infty} \frac{1}{\left(\begin{array}{c}
2 j \\
j
\end{array}\right)} \frac{u^{j}}{j}\left\{\frac{1}{\varepsilon}+\left[5 S_{1}-2 \bar{S}_{1}\right]+\varepsilon\left[\frac{25}{2} S_{1}^{2}-10 S_{1} \bar{S}_{1}-\frac{5}{2} S_{2}+2 \bar{S}_{1}^{2}+2 \bar{S}_{2}\right]\right. \\
& +\varepsilon^{2}\left[\frac{125}{6} S_{1}^{3}-25 S_{1}^{2} \bar{S}_{1}-\frac{25}{2} S_{1} S_{2}+\frac{11}{3} S_{3}+5 S_{2} \bar{S}_{1}+10 S_{1}\left(\bar{S}_{1}^{2}+\bar{S}_{2}\right)\right. \\
& \left.\left.-\frac{4}{3}\left(\bar{S}_{1}^{3}+3 \bar{S}_{1} \bar{S}_{2}+2 \bar{S}_{3}\right)\right]+\mathcal{O}\left(\varepsilon^{3}\right)\right\} \\
& =\frac{2\left(m^{2}\right)^{-2 \varepsilon}}{p^{2}} \frac{(1-y)^{1-2 \varepsilon}}{(1+y)^{1+6 \varepsilon}}\left\{\frac{1}{\varepsilon} \ln y+2 \ln ^{2} y-\zeta_{2}-6 \operatorname{Li}_{2}(-y)-2 \operatorname{Li}_{2}(y)\right. \\
& +\varepsilon\left[24 \mathrm{~S}_{1,2}(-y)+6 \mathrm{~S}_{1,2}\left(y^{2}\right)-8 \mathrm{~S}_{1,2}(y)+\frac{5}{3} \ln 3-4 \zeta_{2} \ln y-11 \zeta_{3}-24 \operatorname{Li}_{3}(-y)-8 \operatorname{Li}_{3}(y)\right] \\
& +\varepsilon^{2}\left[24 \operatorname{Li}_{4}(\omega)-24 \operatorname{Li}_{4}(-\omega)-24 \mathrm{~S}_{1,3}\left(y^{2}\right)+12 \mathrm{~S}_{2,2}\left(y^{2}\right)+64 \mathrm{~S}_{1,3}(y)\right. \\
& +96 \mathrm{~S}_{2,2}(-y)-32 \mathrm{~S}_{2,2}(y)-192 \mathrm{~S}_{1,3}(-y)+52 \operatorname{Li}_{4}(y)+48 \operatorname{Li}_{4}(-y) \\
& \quad-6 \ln \omega\left[2 \mathrm{~S}_{1,2}\left(y^{2}\right)-8 \mathrm{~S}_{1,2}(y)-8 \mathrm{~S}_{1,2}(-y)+7 \zeta_{3}\right]-72 \ln y \operatorname{Li}_{3}(-y)-48 \ln y \operatorname{Li}_{3}(y)
\end{aligned}
$$




$$
\begin{aligned}
& +6 \ln ^{2} \omega\left[2 \mathrm{Li}_{2}(y)-2 \operatorname{Li}_{2}(-y)-3 \zeta_{2}\right]+12 \ln ^{2} y \operatorname{Li}_{2}(y)+18 \ln ^{2} y \operatorname{Li}_{2}(-y) \\
& \left.\left.+4 \ln y \ln ^{3} \omega+\frac{11}{12} \ln ^{4} y-5 \zeta_{2} \ln ^{2} y-26 \zeta_{3} \ln y-57 \zeta_{4}\right]+\mathcal{O}\left(\varepsilon^{3}\right)\right\}
\end{aligned}
$$

where $\omega=(1-y) /(1+y)$, see Eq. (3.5) . The $\varepsilon$-term can be related to that of the result for $J_{011}(1,1,3)$ presented in Ref. [18], whereas the result for the $\varepsilon^{2}$ term is new.

\subsection{Two-loop vertex diagrams}

Consider the two-loop vertex-type diagram $P_{126}$ given in Ref. $[14]^{8}$ (see Fig. 1),

$$
\begin{aligned}
\left(p^{2}\right)^{2} P_{126}= & \sum_{j=1}^{\infty} \frac{1}{\left(\begin{array}{c}
2 j \\
j
\end{array}\right)} \frac{u^{j}}{j^{2}}\left\{\frac{1}{\varepsilon^{2}}+\frac{1}{\varepsilon}\left[-S_{1}-\log (-u)\right]\right. \\
& \left.+\frac{1}{2} \log ^{2}(-u)-S_{1} \log (-u)-\frac{3}{2} S_{2}-\frac{15}{2} S_{1}^{2}+4 S_{1} \bar{S}_{1}+2 \frac{S_{1}}{j}+\mathcal{O}(\varepsilon)\right\} .
\end{aligned}
$$

It corresponds to one of the two-loop contributions to a boson decay into two massless particles, with a massive triangle subloop. Diagrams of such type have been intensively studied for Higgs boson production via gluon fusion [42].

Using our approach, we obtain ${ }^{9}$

$$
\begin{aligned}
\left(p^{2}\right)^{2} P_{126}= & -\frac{1}{2 \varepsilon^{2}} \ln ^{2} y+\frac{1}{\varepsilon}\left[2 \operatorname{Li}_{2}(-y) \ln y-4 \operatorname{Li}_{3}(-y)+\ln ^{2} y \ln (1-y)-\frac{1}{3} \ln ^{3} y-\zeta_{2} \ln y-3 \zeta_{3}\right] \\
& +8 H_{-1,0,0,1}(-y)+2 \mathrm{~S}_{2,2}\left(y^{2}\right)-8 \mathrm{~S}_{2,2}(-y)-8 \mathrm{~S}_{2,2}(y)-4 \operatorname{Li}_{4}(y)-12 \operatorname{Li}_{4}(-y) \\
& -8 \operatorname{Li}_{2}(y) \operatorname{Li}_{2}(-y)-14\left[\operatorname{Li}_{2}(-y)\right]^{2}+28 \mathrm{~S}_{1,2}(-y) \ln y-10 \operatorname{Li}_{3}(-y) \ln y \\
& +7 \operatorname{Li}_{2}(-y) \ln ^{2} y-4 \mathrm{Li}_{2}(-y) \ln y \ln (1-y)-\ln ^{2} y \ln ^{2}(1-y)-\frac{1}{6} \ln ^{4} y \\
& +\frac{2}{3} \ln ^{3} y \ln (1-y)+2 \zeta_{2} \ln y \ln (1-y)-\frac{5}{2} \zeta_{2} \ln ^{2} y+6 \zeta_{3} \ln (1-y)-11 \zeta_{3} \ln y \\
& -6 \operatorname{Li}_{2}(-y) \zeta_{2}-\frac{27}{4} \zeta_{4}+\mathcal{O}(\varepsilon) .
\end{aligned}
$$

Here, the result for the finite part is new. Alternatively, results of such type can be obtained in a different way, using a technique based on Mellin-Barnes contour integrals [46].

Let us consider another two-loop vertex-type diagram shown in Fig. $1, K\left(\nu_{1}, \nu_{2}, \sigma_{1}, \sigma_{2}, \sigma_{3}\right)$, where two external momenta are on shell $\left(p_{1}^{2}=p_{2}^{2}=m^{2}\right)$ and we also put $p_{3}^{2}=p^{2}$. Note

\footnotetext{
${ }^{8}$ The $q^{2}$ from Ref. [14] corresponds to our $p^{2}$, whereas their $z$ corresponds to our $u$. For $P_{126}$ we keep the normalization used in in Ref. [14] for two-loop vertices: each loop integral is divided by $\pi^{2-\varepsilon} m^{-2 \varepsilon} e^{-\gamma_{E} \varepsilon}$, where $\gamma_{E}$ is Euler's constant. Note that it is different from the normalization used in Ref. [41], where each loop is divided by $\mathrm{i} \pi^{2-\varepsilon} \Gamma(1+\varepsilon) \mu^{-2 \varepsilon}$, where $\mu$ is the scale parameter of dimensional regularization [1]. Furthermore, in numerical results presented in Table 1 of Ref. [41] an extra common factor $m^{4} \Gamma(1-2 \varepsilon) / \Gamma^{2}(1-\varepsilon)$ was extracted.

${ }^{9}$ Recently this result was checked numerically by G. Passarino and S. Uccirati with the help of their approach [43]. After fixing a typo in an earlier version of our result, the results are in full agreement. The corrected result below was also confirmed (according to a private communication by R. Bonciani) by R. Bonciani, P. Mastrolia and E. Remiddi [44], using their approach [45].
} 
that $K(1,1,1,1,0)$ appears as one of the master integrals in Ref. [45] (see Eqs. (102)-(106) of $[45])$.

First of all, integration over the momentum of the massless loop (with powers of the propagators equal to $\sigma_{1}$ and $\sigma_{2}$ ) can be easily performed, yielding the corresponding massless propagator to the power $\sigma_{1}+\sigma_{2}-\frac{n}{2}$, times a well-known factor containing $\Gamma$ functions. The resulting integral is nothing but a one-loop triangle function, $J_{2}\left(\nu_{1}, \nu_{2}, \sigma_{1}+\sigma_{2}+\sigma_{3}-\frac{n}{2} ; m\right)$ in the notation of Ref. [10], with two external momenta $\left(p_{1}\right.$ and $\left.p_{2}\right)$ on shell and $\nu_{3}=$ $\sigma_{1}+\sigma_{2}+\sigma_{3}-\frac{n}{2}$. Using the results of Ref. [10], one can see that in this on-shell limit the threepoint integral $J_{2}$ reduces to a two-point function $J^{(2)}$ with two massive propagators (with the powers $\nu_{1}$ and $\left.\nu_{2}\right)$, but with a shifted value of the space-time dimension $\left(n \rightarrow n-2 \nu_{3}\right)$,

$$
\left.J_{2}\left(\nu_{1}, \nu_{2}, \nu_{3} ; m\right)\right|_{p_{1}^{2}=p_{2}^{2}=m^{2}, p_{3} \equiv p}=\pi^{\nu_{3}} \mathrm{i}^{-2 \nu_{3}} \frac{\Gamma\left(n-\nu_{1}-\nu_{2}-2 \nu_{3}\right)}{\Gamma\left(n-\nu_{1}-\nu_{2}-\nu_{3}\right)} J^{(2)}\left(n-2 \nu_{3} ; \nu_{1}, \nu_{2}\right) \text {. }
$$

This is a generalization to arbitrary values of $\nu_{i}$ of the relation found in Ref. [16,47] for $\nu_{1}=\nu_{2}=\nu_{3}=1$.

Using Eq. (4.12), we arrive at the following result for the diagram $K\left(\nu_{1}, \nu_{2}, \sigma_{1}, \sigma_{2}, \sigma_{3} ; p^{2}, m\right)$ (which we normalize by dividing each loop by $\mathrm{i} \pi^{n / 2} \Gamma\left(3-\frac{n}{2}\right)$ ):

$$
\begin{aligned}
K\left(\nu_{1}, \nu_{2},\right. & \left.\sigma_{1}, \sigma_{2}, \sigma_{3} ; p^{2}, m\right) \\
= & \frac{(-1)^{\nu_{1}+\nu_{2}+\sigma_{1}+\sigma_{2}+\sigma_{3}}}{\left(m^{2}\right)^{\nu_{1}+\nu_{2}+\sigma_{1}+\sigma_{2}+\sigma_{3}-n}} \frac{\Gamma\left(\nu_{1}+\nu_{2}+\sigma_{1}+\sigma_{2}+\sigma_{3}-n\right) \Gamma\left(\frac{n}{2}-\sigma_{1}\right) \Gamma\left(\frac{n}{2}-\sigma_{2}\right) \Gamma\left(\sigma_{1}+\sigma_{2}-\frac{n}{2}\right)}{\Gamma\left(\sigma_{1}\right) \Gamma\left(\sigma_{2}\right) \Gamma\left(\nu_{1}+\nu_{2}\right) \Gamma\left(n-\sigma_{1}-\sigma_{2}\right) \Gamma\left(3-\frac{n}{2}\right)} \\
& \times \frac{\Gamma\left(2 n-\nu_{1}-\nu_{2}-2 \sigma_{1}-2 \sigma_{2}-2 \sigma_{3}\right)}{\Gamma\left(\frac{3 n}{2}-\nu_{1}-\nu_{2}-\sigma_{1}-\sigma_{2}-\sigma_{3}\right)} F_{2}\left(\begin{array}{c}
\nu_{1}, \nu_{2}, \nu_{1}+\nu_{2}+\sigma_{1}+\sigma_{2}+\sigma_{3}-n \\
\frac{1}{2}\left(\nu_{1}+\nu_{2}\right), \frac{1}{2}\left(\nu_{1}+\nu_{2}+1\right)
\end{array}\right),
\end{aligned}
$$

where $u=p^{2} / m^{2}$.

For $\nu_{1}=\nu_{2}=\sigma_{1}=\sigma_{2}=1$ and $\sigma_{3}=0$, the parameter $\nu_{3}$ in Eq. (4.12) gets equal to $\varepsilon$, and the function corresponds to a one-loop two-point function in $4-4 \varepsilon$ dimensions (i.e., $\varepsilon \rightarrow 2 \varepsilon)$,

$$
K\left(1,1,1,1,0 ; p^{2}, m\right)=\left(m^{2}\right)^{-2 \varepsilon} \frac{1}{2 \varepsilon^{2}} \frac{\Gamma^{2}(1-\varepsilon) \Gamma(1+2 \varepsilon) \Gamma(2-4 \varepsilon)}{\Gamma(2-2 \varepsilon) \Gamma(2-3 \varepsilon) \Gamma(1+\varepsilon)}{ }_{2} F_{1}\left(\begin{array}{c|c}
1,2 \varepsilon & \frac{u}{4} \\
\frac{3}{2} & \frac{3}{4}
\end{array}\right)
$$

As in the one-loop case (cf. Eqs. (C29)-(C30) of Ref. [47]), the ${ }_{2} F_{1}$ function can be reduced to the type considered in section 2 by means of one of the Kummer relations,

$$
(1-4 \varepsilon){ }_{2} F_{1}\left(\begin{array}{c}
1,2 \varepsilon \\
\frac{3}{2}
\end{array} \mid z\right)=1-4 \varepsilon(1-z){ }_{2} F_{1}\left(\begin{array}{c}
1,1+2 \varepsilon \\
\frac{3}{2}
\end{array} \mid z\right)
$$

All orders of the $\varepsilon$-expansion of the resulting ${ }_{2} F_{1}$ function are given in (see also in Refs. [16, 30]). For another special case, $K(1,1,1,1,1)$, the parameter $\nu_{3}$ in Eq. (4.12) is equal to $1+\varepsilon$, and the function corresponds to a one-loop two-point function in $2-4 \varepsilon$ dimensions. In this case, we directly get the ${ }_{2} F_{1}$ function of the type (2.10), with $\varepsilon \rightarrow 2 \varepsilon$. 
Analytic continuation in terms of the Nielsen polylogarithms was discussed in section 2.2 of Ref. [16]. In the case of $K(1,1,1,1,0)$, we get

$$
\begin{aligned}
K(1,1,1,1,0 ; & \left.p^{2}, m\right)=\left(m^{2}\right)^{-2 \varepsilon} \frac{1}{2 \varepsilon^{2}} \frac{\Gamma^{2}(1-\varepsilon) \Gamma(1+2 \varepsilon) \Gamma(1-4 \varepsilon)}{\Gamma(1-2 \varepsilon) \Gamma(1-3 \varepsilon) \Gamma(1+\varepsilon)} \frac{1}{(1-2 \varepsilon)(1-3 \varepsilon)} \\
& \times\left\{1-\frac{(1+y)^{1-4 \varepsilon}}{2(1-y)}\left(1-y^{4 \varepsilon}\right)-2 \varepsilon \frac{(1+y)^{1-4 \varepsilon}}{y^{-2 \varepsilon}(1-y)} \sum_{j=0}^{\infty}(-4 \varepsilon)^{j}\right. \\
& \left.\times \sum_{p=0}^{j-1} \frac{\ln ^{p} y}{2^{p} p !} \sum_{k=1}^{j-p}(-2)^{-k}\left[\mathrm{~S}_{k, j+1-k-p}(-y)-(-1)^{p} \mathrm{~S}_{k, j+1-k-p}\left(-y^{-1}\right)\right]\right\}
\end{aligned}
$$

where the generalized polylogarithms $\mathrm{S}_{k, p}\left(-y^{-1}\right)$ can be expressed in terms of inverse argument by means of the standard formulae given in Ref. [3]. In this way, we have obtained results for all coefficients of the $\varepsilon$-expansion of $K(1,1,1,1,0)$. The first three coefficients coincide with those given in Eqs. (104)-(106) of Ref. [45].

\subsection{Three-loop vacuum diagram $D_{4}$}

Consider a three-loop vacuum diagram with two different masses shown in Fig. (11). Such an integral with equal masses $(M=m)$ enters as a master integral in Avdeev's package [49] and MATAD [50]. Although there is no similar package for three-loop vacuum diagrams with two different masses, it is clear that such configuration (with unit powers of the propagators) will remain one of the master integrals in this more general case.

This integral can be calculated by integrating over the momentum $p$ the off-shell twoloop diagram $F_{10101}$ (also shown in Fig. 1) with a massive propagator containing an arbitrary mass $M$ (see Eq. (4.7) in [16]),

$$
D_{4}(1,1,1,1,1,1 ; u)=\frac{1}{\mathrm{i} \pi^{n / 2}} \int \frac{\mathrm{d}^{n} p}{p^{2}-M^{2}} F_{10101}\left(p^{2}, m\right)
$$

where $u=M^{2} / m^{2}$. The result of this integration can be presented in the following form:

$$
\begin{aligned}
\left(m^{2}\right)^{3 \varepsilon} & (1-\varepsilon)(1-2 \varepsilon) D_{4}(1,1,1,1,1,1 ; u) \\
& =-\frac{u^{1-\varepsilon}}{\varepsilon(1-\varepsilon)(1+\varepsilon)(1+2 \varepsilon)}{ }_{4} F_{3}\left(\begin{array}{c}
1,1+\varepsilon, 1+\varepsilon, 1+2 \varepsilon \\
\frac{3}{2}+\varepsilon, 2+\varepsilon, 2-\varepsilon
\end{array}\right. \\
& +\frac{u \Gamma(1-\varepsilon) \Gamma^{2}(1+2 \varepsilon) \Gamma(1+3 \varepsilon)}{\varepsilon(1+2 \varepsilon)(1+4 \varepsilon) \Gamma^{2}(1+\varepsilon) \Gamma(1+4 \varepsilon)}{ }_{4} F_{3}\left(\begin{array}{c}
1,1+2 \varepsilon, 1+2 \varepsilon, 1+3 \varepsilon \\
\frac{3}{2}+2 \varepsilon, 2,2+2 \varepsilon
\end{array}\right. \\
& +\frac{u^{1-\varepsilon}}{2 \varepsilon^{2}(1+\varepsilon)}{ }_{3} F_{2}\left(\begin{array}{c}
1,1+\varepsilon, 1+\varepsilon \\
\frac{3}{2}, 2+\varepsilon
\end{array}\right) \\
& \left.-u^{1-2 \varepsilon} \frac{u}{4}\right)-\frac{u}{2 \varepsilon^{2}(1+2 \varepsilon)^{2}}{ }_{3} F_{2}\left(\begin{array}{c}
1,1+2 \varepsilon, 1+2 \varepsilon \\
\frac{3}{2}+\varepsilon, 2+2 \varepsilon
\end{array}\right) \\
& +\frac{u \Gamma(1-\varepsilon) \Gamma(1+2 \varepsilon)}{4 \varepsilon^{2} \Gamma(1+\varepsilon)}{ }_{3} F_{2}\left(\begin{array}{c}
1,1,1+\varepsilon \\
\frac{3}{2}, 2
\end{array}\right)
\end{aligned}
$$


where, as before, $u=4 z=4 \sin ^{2} \frac{\theta}{2}$. All hypergeometric functions occurring in Eq. (4.17) belong to the type considered in section 2. Applying the results for the $\varepsilon$-expansion of these functions, we arrive at

$$
\begin{aligned}
& \left(m^{2}\right)^{3 \varepsilon}(1-\varepsilon)(1-2 \varepsilon) D_{4}(1,1,1,1,1,1 ; u) \\
& =\frac{2 \zeta_{3}}{\varepsilon}-9 \zeta_{4}+\sum_{j=1}^{\infty} \frac{u^{j}}{\left(\begin{array}{c}
2 j \\
j
\end{array}\right)}\left\{-\frac{1}{2 j^{2}} \ln ^{2} u+\frac{3}{j^{3}} \ln u-\frac{5}{j^{4}}-\frac{1}{j^{2}} \zeta_{2}\right. \\
& \left.\quad+\frac{4}{j^{3}} S_{1}-\frac{4}{j^{3}} \bar{S}_{1}+\frac{2}{j^{2}} S_{1}^{2}-\frac{4}{j^{2}} S_{1} \bar{S}_{1}-\frac{1}{j^{2}} S_{2}+\frac{2}{j^{2}}\left(\bar{S}_{1}^{2}+\bar{S}_{2}\right)+\mathcal{O}(\varepsilon)\right\} \\
& =\frac{2 \zeta_{3}}{\varepsilon}+2 \mathrm{Ls}_{4}^{(1)}(\theta)+8 l_{\theta}\left[\mathrm{Cl}_{3}(\theta)-\zeta_{3}\right]-2 \theta \mathrm{Ls}_{3}(\theta)-6\left[\mathrm{Ls}_{2}(\theta)\right]^{2}+\frac{1}{12} \theta^{4}-\frac{1}{2} \zeta_{2} \theta^{2}-9 \zeta_{4}+\mathcal{O}(\varepsilon) .
\end{aligned}
$$

Using analytic continuation described in section 3, we can also present this result in terms of the variable $y$ defined in (3.2),

$$
\begin{aligned}
& \left(m^{2}\right)^{3 \varepsilon}(1-\varepsilon)(1-2 \varepsilon) D_{4}(1,1,1,1,1,1 ; u) \\
& =\frac{2 \zeta_{3}}{\varepsilon}+\frac{1}{4} \ln ^{2} u \ln ^{2} y+\ln u\left[6 \operatorname{Li}_{3}(y)-6 \ln y \operatorname{Li}_{2}(y)+\frac{1}{2} \ln ^{3} y-3 \ln ^{2} y \ln (1-y)-6 \zeta_{3}\right] \\
& \quad+4 \operatorname{Li}_{4}(y)-4 \mathrm{~S}_{2,2}(y)+6\left[\operatorname{Li}_{2}(y)\right]^{2}-4 \ln (1-y) \operatorname{Li}_{3}(y)+12 \ln y \ln (1-y) \operatorname{Li}_{2}(y) \\
& \quad-3 \ln ^{2} y \operatorname{Li}_{2}(y)+5 \ln ^{2} y \ln ^{2}(1-y)-\frac{7}{3} \ln ^{3} y \ln (1-y)+\frac{1}{4} \ln ^{4} y \\
& \quad-12 \zeta_{2} \operatorname{Li}_{2}(y)-8 \zeta_{2} \ln y \ln (1-y)+\frac{3}{2} \zeta_{2} \ln ^{2} y+4 \zeta_{3} \ln (1-y)+3 \zeta_{4}+\mathcal{O}(\varepsilon) .
\end{aligned}
$$

One should remember however, that in this case the variable $y$ becomes complex (although all imaginary parts should cancel for real masses), whereas Eq. (4.18) is explicitly real.

As a non-trivial check on these results we consider two particular values, $M^{2}=m^{2}$ and $M^{2}=0$. In the first case $\left(\theta=\frac{\pi}{3}\right)$, we reproduce the known result (see in Ref. [23]) for the master integral $\left.\mathbf{D}_{4} \equiv D_{4}(1,1,1,1,1,1 ; u)\right|_{u \rightarrow 1}$,

$$
\left.\left(m^{2}\right)^{3 \varepsilon}(1-\varepsilon)(1-2 \varepsilon) D_{4}(1,1,1,1,1,1 ; u)\right|_{u \rightarrow 1}=\frac{2 \zeta_{3}}{\varepsilon}-\frac{77}{12} \zeta_{4}-6\left[\operatorname{Ls}_{2}\left(\frac{\pi}{3}\right)\right]^{2}+\mathcal{O}(\varepsilon)
$$

In the second case, $M^{2}=0(\theta=0, y \rightarrow 1)$, the result for the master integral $B_{M}$ is reproduced (which was first calculated in Ref. [51]),

$$
\left.\left(m^{2}\right)^{3 \varepsilon}(1-\varepsilon)(1-2 \varepsilon) D_{4}(1,1,1,1,1,1 ; u)\right|_{u \rightarrow 0}=\left(m^{2}\right)^{3 \varepsilon}(1-\varepsilon)(1-2 \varepsilon) B_{M}=\frac{2 \zeta_{3}}{\varepsilon}-9 \zeta_{4}+\mathcal{O}(\varepsilon) .
$$

In a similar manner, analytical results can be deduced for other diagrams with two different mass scales, like $D_{3}$ and $E_{3}$ (for notations, see in Ref. [16]). To our knowledge, this is the first example of the calculation of the finite part of a three-loop vacuum diagram with six internal lines with two different mass scales. In previous publications [52], only the divergent parts of some three-loop vacuum integrals have been analyzed. 


\section{Conclusion}

In this paper, we have studied the multiple inverse binomial sums of the type (1.1), for arbitrary values of the variable $u$. Our analysis was based on the connection between these sums and terms of the expansion of certain hypergeometric functions with respect to the parameter $\varepsilon$. Using known results for the hypergeometric functions, together with the integral representation (2.32), we have obtained a number of new analytical results up to the weight 4, Eqs. (2.19)-(2.27), (2.35)-(2.39) and (2.43)-(2.44). Moreover, in some cases like (2.33) -(2.34) the results can be obtained for an arbitrary weight. Constructing analytical continuation of the obtained results, we have expressed them in terms of the generalized polylogarithms (3.7)-(3.13). In the cases considered, only one new function was needed, in addition to the basis of Nielsen polylogarithms. As such function one can take, e.g., the harmonic polylogarithm $H_{-1,0,0,1}(-y)$, where the variable $y$ is given in Eq. (3.2).

This approach allowed us to construct some terms of the $\varepsilon$-expansion of the generalized hypergeometric function ${ }_{P+1} F_{P}$ (2.30) and obtain new analytical results for higher terms of the $\varepsilon$-expansion of some one- and two-loop propagator-type (see Eqs. (4.6), (4.8) and (4.9)) and vertex-type (see Eqs. (4.3) and (4.11)) diagrams depending on one dimensionless variable $u=p^{2} / m^{2}$. As a by-product, we have analytically proven the earlier published results for the three-loop vacuum integrals, the finite part of $\mathbf{D}_{\mathbf{4}}$ and the $\varepsilon$-part of $\mathbf{D}_{\mathbf{3}}$ and $\mathbf{E}_{\mathbf{3}}$, corresponding to the particular value of $u=1\left(z=\frac{1}{4}\right)$, see Sections $4.3,4.5$ and 4.6 in Ref. [16] and Refs. [21,23,61]. These integrals enter as master integrals in Avdeev's package [49] and MATAD [50]. Moreover, the developed technique is useful in the calculation of two-loop vertex-type diagrams $[45,48]$.

Acknowledgements. We are grateful to F. Jegerlehner, A. Kotikov, M. Spira, V.A. Smirnov and O.V. Tarasov for useful discussions. We would like to thank R. Delbourgo and J. Fleischer for their interest in our work, and J. Gluza for checks of some formulae. We are very thankful to G. Passarino and S. Uccirati for an independent numerical check of some expressions from sections 3 and 4 which helped to identify typos in earlier versions of Eqs. (3.11) and (4.11). This research was supported in part by INTAS-CERN grant No. 99-0377 and by the Australian Research Council grant No. A00000780.

\section{A Harmonic polylogarithms of complex arguments}

Here we collect some properties of the harmonic polylogarithms introduced in [4]. Let us define a $w$-dimensional vector $\vec{a}=(a, \vec{b})$, where $a$ is the leftmost component of $\vec{a}$, while $\vec{b}$ stands for the vector of the remaining $(w-1)$ components. The harmonic polylogarithms of weight $w$ are then defined as follows:

$$
\mathrm{H}_{\vec{a}}(y)=\int_{0}^{y} \mathrm{~d} x \mathrm{f}(a ; x) \mathrm{H}_{\vec{b}}(x),
$$


where the three rational fractions $\mathrm{f}(a ; x)$ are given by

$$
\mathrm{f}(+1 ; x)=\frac{1}{1-x}, \quad \mathrm{f}(0 ; x)=\frac{1}{x}, \quad \mathrm{f}(-1 ; x)=\frac{1}{1+x} .
$$

The derivatives can be written in a compact form

$$
\frac{\mathrm{d}}{\mathrm{d} y} \mathrm{H}_{\vec{a}}(y)=\mathrm{f}(a ; y) \mathrm{H}_{\vec{b}}(y) .
$$

Let us put $y=e^{\mathrm{i} \theta}$, so that

$$
\mathrm{H}_{\vec{a}}\left(e^{\mathrm{i} \theta}\right)=\mathrm{H}_{\vec{a}}(1)+\mathrm{i} \int_{0}^{\theta} \mathrm{d} \phi e^{\mathrm{i} \phi} \mathrm{f}\left(a ; e^{\mathrm{i} \phi}\right) \mathrm{H}_{\vec{b}}\left(e^{\mathrm{i} \phi}\right),
$$

where

$$
\begin{aligned}
e^{\mathrm{i} \phi} \mathrm{f}\left(+1 ; e^{\mathrm{i} \phi}\right) & =\frac{e^{\mathrm{i}(\pi+\phi) / 2}}{2 \sin \frac{\phi}{2}}=-\frac{1}{2}\left(1-\mathrm{i} \cot \frac{\phi}{2}\right) \\
e^{\mathrm{i} \phi} \mathrm{f}\left(0 ; e^{\mathrm{i} \phi}\right) & =1 \\
e^{\mathrm{i} \phi} \mathrm{f}\left(-1 ; e^{\mathrm{i} \phi}\right) & =\frac{e^{\mathrm{i} \phi / 2}}{2 \cos \frac{\phi}{2}}=\frac{1}{2}\left(1+\mathrm{i} \tan \frac{\phi}{2}\right) .
\end{aligned}
$$

Consider, for example, the harmonic polylogarithm $H_{-1,0,0,1}(y)$ given in Eq. (2.46). Using the decomposition of $\mathrm{Li}_{3}\left(e^{\mathrm{i} \phi}\right)$ into the real and imaginary parts [2] and integrating by parts, we obtain expressions in terms of Clausen's and generalized log-sine functions,

$$
\begin{aligned}
\mathrm{H}_{-1,0,0,1}\left(e^{\mathrm{i} \theta}\right)= & \mathrm{H}_{-1,0,0,1}(1)+\frac{\mathrm{i}}{2} \int_{0}^{\theta} \mathrm{d} \phi \operatorname{Li}_{3}\left(e^{\mathrm{i} \phi}\right)\left(1+\mathrm{i} \frac{\sin \frac{\phi}{2}}{\cos \frac{\phi}{2}}\right) \\
= & \mathrm{H}_{-1,0,0,1}(1)-\frac{1}{96} \theta^{2}(2 \pi-\theta)^{2}+\Phi(\theta)+\ln \left(2 \cos \frac{\theta}{2}\right) \mathrm{Cl}_{3}(\theta)-\zeta_{3} \ln 2 \\
& +\mathrm{i} \ln \left(2 \cos \frac{\theta}{2}\right) \mathrm{Gl}_{3}(\theta)+\frac{1}{2} \mathrm{i}\left[\mathrm{Cl}_{4}(\theta)+\mathrm{Cl}_{4}(\pi-\theta)\right] \\
& -\mathrm{i}\left[\mathrm{Gl}_{2}(\theta) \mathrm{Cl}_{2}(\pi-\theta)+\frac{1}{2}(\pi-\theta) \mathrm{Cl}_{3}(\pi-\theta)-\frac{1}{2} \pi \mathrm{Cl}_{3}(\pi)\right]
\end{aligned}
$$

where

$$
\mathrm{Cl}_{3}(\pi)=-\frac{3}{4} \zeta_{3}, \quad \mathrm{Gl}_{2}(\theta)=\zeta_{2}-\frac{1}{2} \pi \theta+\frac{1}{4} \theta^{2}, \quad \mathrm{Gl}_{3}(\theta)=\frac{1}{12} \theta(\pi-\theta)(2 \pi-\theta),
$$

and $\Phi(\theta)$ is defined in Eq. (2.40).

In this paper, we have also used the following relations:

$$
\begin{aligned}
H_{-1,0,0,1}\left(-y^{-1}\right)= & H_{-1,0,0,1}(-y)+\operatorname{Li}_{4}\left(-y^{-1}\right)+\operatorname{Li}_{4}(y)-\operatorname{Li}_{3}(y) \ln y-\zeta_{2} \operatorname{Li}_{2}(1-y) \\
& +\frac{1}{2} \ln ^{2} y \operatorname{Li}_{2}(y)+\frac{1}{6} \ln ^{3} y \ln (1-y)-\frac{1}{8} \zeta_{4}-\mathrm{i} \sigma \pi \frac{3}{4} \zeta_{3}
\end{aligned}
$$


and

$$
H_{-1,0,0,1}(y)+H_{-1,0,0,1}(-y)=\operatorname{Li}_{2}(y) \operatorname{Li}_{2}(-y)+\ln (1+y) \operatorname{Li}_{3}(y)+\ln (1-y) \operatorname{Li}_{3}(-y) .
$$

The following two representations of $H_{-1,0,0,1}(y)$ are also useful:

$$
\begin{aligned}
& H_{-1,0,0,1}(y)=\operatorname{Li}_{4}(y)-\frac{3}{4} \zeta_{3} \ln (1+y)+\frac{1}{2} \int_{0}^{1} \frac{\mathrm{d} x \ln ^{2} x \ln (1-x y)}{1+x}, \\
& H_{-1,0,0,1}(y)=-\frac{3}{4} \zeta_{3} \ln (1+y)+\frac{1}{16} \sum_{l=1}^{\infty} \frac{y^{l}}{l}\left[\psi^{\prime \prime}\left(\frac{l+1}{2}\right)-\psi^{\prime \prime}\left(\frac{l}{2}\right)\right] .
\end{aligned}
$$

Instead of $\Phi(\theta)$, one could also introduce another function. One of such possibilities is to consider a generalization of the Glashier function. Let us recall that the real part of $\operatorname{Li}_{j}\left(e^{\mathrm{i} \theta}\right)$ can be presented as $^{10}$

$$
\mathrm{Li}_{j}(1, \theta)=\frac{(-1)^{j-1}}{2(j-2) !} \int_{0}^{1} \frac{\mathrm{d} \xi}{\xi} \ln ^{j-2} \xi \ln \left(1-2 \xi \cos \theta+\xi^{2}\right)= \begin{cases}\mathrm{Cl}_{j}(\theta), & j \text { odd } \\ \mathrm{Gl}_{j}(\theta), & j \text { even }\end{cases}
$$

where $\mathrm{Cl}_{j}(\theta)$ and $\mathrm{Gl}_{j}(\theta)$ are Clausen and Glashier functions, respectively. In particular, $\mathrm{Gl}_{j}(\theta)$ is just a polynomial in $\theta$.

A possible non-trivial generalization of $\mathrm{Gl}_{j}(\theta)$ (for even $j$ ) could be

$$
\mathrm{Gl}_{j}(\theta ; a)=-\frac{1}{2(j-2) !} \int_{0}^{1} \frac{\mathrm{d} \xi}{\xi+a} \ln ^{j-2} \xi \ln \left(1-2 \xi \cos \theta+\xi^{2}\right), \quad j \text { even, }
$$

so that $\mathrm{Gl}_{j}(\theta ; 0)=\mathrm{Gl}_{j}(\theta)$. In particular,

$$
\mathrm{Gl}_{2}(\theta ; a)=-\ln b \ln \frac{a+1}{a}+\mathrm{Li}_{2}\left(\frac{a+1}{b}, \tilde{\theta}\right)-\mathrm{Li}_{2}\left(\frac{a}{b}, \tilde{\theta}\right),
$$

where

$$
\cos \widetilde{\theta}=\frac{a+\cos \theta}{\sqrt{1+2 a \cos \theta+a^{2}}}
$$

so that

$$
a=\frac{\sin (\theta-\widetilde{\theta})}{\sin \widetilde{\theta}}, \quad b=\sqrt{1+2 a \cos \theta+a^{2}}=\frac{\sin \theta}{\sin \widetilde{\theta}} .
$$

Using Eqs. (17) and (18) on p. 293 of [2], we can see that the general result (A.12) simplifies in the case $a=1\left(\widetilde{\theta}=\frac{\pi}{2}\right)$,

$$
\mathrm{Gl}_{2}(\theta ; 1)=-\frac{1}{4} \mathrm{Li}_{2}\left(\cos ^{2} \frac{\theta}{2}\right)+\frac{1}{8}(\pi-\theta)^{2}-\frac{1}{2} \ln ^{2} 2 .
$$

\footnotetext{
${ }^{10}$ We use the standard notation $\operatorname{Li}_{j}(r, \theta)=\operatorname{Re}\left[\operatorname{Li}_{j}\left(r e^{\mathrm{i} \theta}\right)\right]$ (see in Ref. [2]).
} 
Then, let us consider

$$
\mathrm{Gl}_{4}(\theta ; 1)=-\frac{1}{4} \int_{0}^{1} \frac{\mathrm{d} \xi}{\xi+1} \ln ^{2} \xi \ln \left(1-2 \xi \cos \theta+\xi^{2}\right) .
$$

For $\theta=0$ we get

$$
\mathrm{Gl}_{4}(0 ; 1)=U_{3,1}-\zeta_{4}
$$

where $U_{3,1}$ is the alternating two-fold Euler sum considered in [22,23] (see also Ref. [53]),

$$
U_{3,1}=-2 \operatorname{Li}_{4}\left(\frac{1}{2}\right)+\frac{1}{2} \zeta_{4}-\frac{1}{12} \ln ^{4} 2+\frac{1}{2} \zeta_{2} \ln ^{2} 2 .
$$

For general $\theta$, one can see that $\mathrm{Gl}_{4}(\theta ; 1)$ is related to the real part of a harmonic polylogarithm,

$$
\operatorname{Re} H_{-1,0,0,1}\left(e^{\mathrm{i} \theta}\right)=\mathrm{Gl}_{4}(\theta)-\mathrm{Gl}_{4}(\theta ; 1)-\frac{3}{4} \zeta_{3} \ln \left(2 \cos \frac{\theta}{2}\right) .
$$

In particular, the function $\Phi(\theta)$ can be presented as

$$
\Phi(\theta)=-\mathrm{Gl}_{4}(\theta ; 1)+\mathrm{Gl}_{4}(0 ; 1)+\frac{7}{4} \zeta_{3} \ln 2-\frac{1}{96} \theta^{2}(2 \pi-\theta)^{2}-\left[\mathrm{Cl}_{3}(\theta)-\mathrm{Cl}_{3}(\pi)\right] \ln \left(2 \cos \frac{\theta}{2}\right),
$$

where $\mathrm{Cl}_{3}(\pi)=-\frac{3}{4} \zeta_{3}$.

Using the symmetry property of $\Phi(\theta)$, Eq. (2.41), we get

$$
\begin{aligned}
\mathrm{Gl}_{4}(\theta ; 1)+\mathrm{Gl}_{4}(\pi-\theta ; 1)= & \frac{11}{8} \zeta_{4}-\frac{1}{48} \theta^{2}(\pi-\theta)^{2}-\mathrm{Cl}_{2}(\theta) \mathrm{Cl}_{2}(\pi-\theta) \\
& -\left[\mathrm{Cl}_{3}(\theta)-\mathrm{Cl}_{3}(\pi)\right] \ln \left(2 \cos \frac{\theta}{2}\right) \\
& -\left[\mathrm{Cl}_{3}(\pi-\theta)-\mathrm{Cl}_{3}(\pi)\right] \ln \left(2 \sin \frac{\theta}{2}\right)
\end{aligned}
$$

For special values of $\theta$, Eq. (A.20) yields

$$
\begin{aligned}
\mathrm{Gl}_{4}(\pi ; 1) & =-U_{3,1}+\frac{19}{8} \zeta_{4}-\frac{7}{4} \zeta_{3} \ln 2 \\
\mathrm{Gl}_{4}\left(\frac{\pi}{2} ; 1\right) & =\frac{161}{256} \zeta_{4}-\frac{21}{64} \zeta_{3} \ln 2-G^{2} \\
\mathrm{Gl}_{4}\left(\frac{\pi}{3} ; 1\right)+\mathrm{Gl}_{4}\left(\frac{2 \pi}{3} ; 1\right) & =\frac{277}{216} \zeta_{4}-\frac{13}{24} \zeta_{3} \ln 3-\frac{2}{3}\left[\mathrm{Cl}_{2}\left(\frac{\pi}{3}\right)\right]^{2}
\end{aligned}
$$

where $G$ is the Catalan constant. Moreover, with the help of PSLQ algorithm [54] one can obtain results for $\mathrm{Gl}_{4}\left(\frac{\pi}{3} ; 1\right)$ and $\mathrm{Gl}_{4}\left(\frac{2 \pi}{3} ; 1\right)$ separately,

$$
\begin{aligned}
\mathrm{Gl}_{4}\left(\frac{\pi}{3} ; 1\right) & =\frac{259}{108} \zeta_{4}-\frac{13}{24} \zeta_{3} \ln 3-\frac{1}{3}\left[\mathrm{Cl}_{2}\left(\frac{\pi}{3}\right)\right]^{2}+\frac{1}{4} \pi \mathrm{Ls}_{3}\left(\frac{2 \pi}{3}\right)-\frac{3}{8} \operatorname{Ls}_{4}^{(1)}\left(\frac{2 \pi}{3}\right) \\
\mathrm{Gl}_{4}\left(\frac{2 \pi}{3} ; 1\right) & =-\frac{241}{216} \zeta_{4}-\frac{1}{3}\left[\mathrm{Cl}_{2}\left(\frac{\pi}{3}\right)\right]^{2}-\frac{1}{4} \pi \mathrm{Ls}_{3}\left(\frac{2 \pi}{3}\right)+\frac{3}{8} \mathrm{Ls}_{4}^{(1)}\left(\frac{2 \pi}{3}\right)
\end{aligned}
$$

We would also like to discuss the analytical continuation of $\operatorname{Lsc}_{2,3}(\theta)$ (see Eq. (2.14)),

$$
\operatorname{Lsc}_{2,3}(\theta)=-\int_{0}^{\theta} \mathrm{d} \phi \ln \left|2 \sin \frac{\phi}{2}\right| \ln ^{2}\left|2 \cos \frac{\phi}{2}\right| .
$$


If we introduce a variable $z=e^{\mathrm{i} \sigma \phi}$, we see that

$$
\ln \left(2 \sin \frac{\phi}{2}\right) \leftrightarrow \ln (1-z)-\frac{1}{2} \ln z+\frac{1}{2} \mathrm{i} \sigma \pi, \quad \ln \left(2 \cos \frac{\phi}{2}\right) \leftrightarrow \ln (1+z)-\frac{1}{2} \ln z
$$

and the analytical continuation of $\operatorname{Lsc}_{2,3}(\theta)$ is given by

$$
\mathrm{i} \sigma \operatorname{Lsc}_{2,3}(\theta)=\int_{y}^{1} \frac{\mathrm{d} z}{z}\left\{\ln (1-z)-\frac{1}{2} \ln z+\frac{1}{2} \mathrm{i} \sigma \pi\right\}\left[\ln ^{2}(1+z)-\ln z \ln (1+z)+\frac{1}{4} \ln ^{2} z\right],
$$

with $y$ defined in (3.1). This integral can be calculated by using the following relations

$$
\begin{aligned}
\int_{0}^{y} \frac{\mathrm{d} z}{z} \ln (1+z) \ln (1-z) \ln z= & \mathrm{S}_{2,2}(y)+\mathrm{S}_{2,2}(-y)-\frac{1}{4} \mathrm{~S}_{2,2}\left(y^{2}\right) \\
& +\ln y\left[\frac{1}{2} \mathrm{~S}_{1,2}\left(y^{2}\right)-\mathrm{S}_{1,2}(y)-\mathrm{S}_{1,2}(-y)\right], \\
\int_{0}^{y} \frac{\mathrm{d} z}{z} \ln ^{2}(1 \mp z) \ln (1 \pm z)= & 2 \ln (1 \pm y) \mathrm{S}_{1,2}( \pm y)-2 \mathrm{H}_{-1,0,1,1}( \pm y),
\end{aligned}
$$

where

$$
\mathrm{H}_{-1,0,1,1}(y) \equiv \int_{0}^{y} \frac{\mathrm{d} z}{1+z} \mathrm{~S}_{1,2}(z)
$$

is another harmonic polylogarithm [19]. We note that

$$
\begin{aligned}
\mathrm{H}_{-1,0,1,1}( \pm y)=\frac{1}{4} \mathrm{~S}_{1,3}\left(y^{2}\right)-\mathrm{S}_{1,3}(\mp y)+ & \ln (1 \pm y) \mathrm{S}_{1,2}( \pm y) \pm \frac{1}{12} \int_{0}^{y} \frac{\mathrm{d} z}{z} \ln ^{3}\left(\frac{1-z}{1+z}\right) \\
\mathrm{H}_{-1,0,1,1}(y)+\mathrm{H}_{-1,0,1,1}(-y)= & \frac{1}{2} \mathrm{~S}_{1,3}\left(y^{2}\right)-\mathrm{S}_{1,3}(y)-\mathrm{S}_{1,3}(-y) \\
& +\ln (1+y) \mathrm{S}_{1,2}(y)+\ln (1-y) \mathrm{S}_{1,2}(-y)
\end{aligned}
$$

Therefore, the analytic continuation of $\operatorname{Lsc}_{2,3}(\theta)$ reads

$$
\begin{aligned}
\mathrm{i} \sigma \operatorname{Lsc}_{2,3}(\theta)= & \mathrm{S}_{2,2}(y)-\frac{1}{4} \mathrm{~S}_{2,2}\left(y^{2}\right)-2 \mathrm{~S}_{1,3}(y)+\frac{1}{2} \mathrm{~S}_{1,3}\left(y^{2}\right)+\frac{1}{2} \operatorname{Li}_{4}(y)+\operatorname{Li}_{4}(-y) \\
& +\ln y\left[\frac{1}{2} \mathrm{~S}_{1,2}\left(y^{2}\right)-\mathrm{S}_{1,2}(y)-\frac{1}{2} \operatorname{Li}_{3}(y)-\mathrm{Li}_{3}(-y)\right] \\
& +\ln ^{2} y\left[\frac{1}{4} \operatorname{Li}_{2}(y)+\frac{1}{2} \operatorname{Li}_{2}(-y)\right]+\frac{1}{32} \ln ^{4} y-\frac{3}{16} \zeta_{4}-\frac{1}{6} \int_{0}^{y} \frac{\mathrm{d} z}{z} \ln ^{3}\left(\frac{1-z}{1+z}\right) \\
& -\mathrm{i} \sigma \pi\left\{\mathrm{S}_{1,2}(-y)-\frac{1}{2} \operatorname{Li}_{3}(-y)+\frac{1}{2} \ln y \operatorname{Li}_{2}(-y)+\frac{1}{24} \ln ^{3} y-\frac{1}{2} \zeta_{3}\right\} .
\end{aligned}
$$

Note that the integral occurring in Eqs. (A.30) and (A.32) is directly related to the integral in Eq. (2.15), and it can be calculated in terms of polylogarithms,

$$
\begin{aligned}
\int_{0}^{y} \frac{\mathrm{d} z}{z} \ln ^{3}\left(\frac{1-z}{1+z}\right)= & -\frac{45}{4} \zeta_{4}+6\left[\operatorname{Li}_{4}(\omega)-\operatorname{Li}_{4}(-\omega)\right]-6 \ln \omega\left[\operatorname{Li}_{3}(\omega)-\operatorname{Li}_{3}(-\omega)\right] \\
& +3 \ln ^{2} \omega\left[\operatorname{Li}_{2}(\omega)-\operatorname{Li}_{2}(-\omega)\right]+\ln ^{3} \omega \ln y
\end{aligned}
$$


where $\omega=(1-y) /(1+y)$ (see Eq. (3.5)). Therefore, $\mathrm{H}_{-1,0,1,1}( \pm y)$ reduces to polylogarithms, as well as the analytic continuation of $\operatorname{Lsc}_{2,3}(\theta)$. We note that the imaginary part on the r.h.s. of Eq. A.32 gets cancelled in the results for the corresponding sums.

The following relations are useful for the transformations:

$$
\begin{aligned}
\mathrm{Li}_{2}(\omega)-\mathrm{Li}_{2}(-\omega)= & -\mathrm{Li}_{2}(y)+\mathrm{Li}_{2}(-y)-\ln \omega \ln y+\frac{3}{2} \zeta_{2} \\
\mathrm{Li}_{3}(\omega)-\mathrm{Li}_{3}(-\omega)= & \frac{1}{2} \mathrm{~S}_{1,2}\left(y^{2}\right)-2 \mathrm{~S}_{1,2}(y)-2 \mathrm{~S}_{1,2}(-y)-\ln \omega\left[\mathrm{Li}_{2}(y)-\mathrm{Li}_{2}(-y)\right] \\
& -\frac{1}{2} \ln ^{2} \omega \ln y+\frac{3}{2} \zeta_{2} \ln \omega+\frac{7}{4} \zeta_{3} .
\end{aligned}
$$

\section{B Additional identities between inverse binomial sums}

As we have seen, the $\varepsilon$-expansion of the hypergeometric functions produces series of the type (1.1). One can use certain properties of hypergeometric functions to get relations between the sums (1.1) involved in the $\varepsilon$-expansion.

Let us consider Eq. (B.18) of Ref. [16] (which follows from Eq. (22) on p. 498 of [31]),

$$
{ }_{3} F_{2}\left(\begin{array}{c}
1+a_{1} \varepsilon, 1+a_{2} \varepsilon, 1+\frac{1}{2}\left(a_{1}+a_{2}\right) \varepsilon \\
\frac{3}{2}+\frac{1}{2}\left(a_{1}+a_{2}\right) \varepsilon, 2+\left(a_{1}+a_{2}\right) \varepsilon
\end{array} \mid z\right)=(1-z)\left[{ }_{2} F_{1}\left(\begin{array}{c}
1+\frac{1}{2} a_{1} \varepsilon, 1+\frac{1}{2} a_{2} \varepsilon \\
\frac{3}{2}+\frac{1}{2}\left(a_{1}+a_{2}\right) \varepsilon
\end{array} \mid z\right)\right]^{2} .
$$

It reduces the given ${ }_{3} F_{2}$ function to a square of the ${ }_{2} F_{1}$ function. Substituting the $\varepsilon$ expansions of the ${ }_{3} F_{2}$ and ${ }_{2} F_{1}$ functions into (B.1), we obtain the following relations between the sums:

$$
\begin{aligned}
& \Sigma_{-;-; 2}^{-;-}(u)=\frac{4-u}{2 u}\left[\Sigma_{-;-; 1}^{-;-}(u)\right]^{2} \\
& \Sigma_{1 ;-; 2}^{1 ;-}(u)-\Sigma_{-; 1 ; 2}^{-; 1}(u)-\Sigma_{-;-; 3}^{-;-}(u)=\frac{4-u}{u} \Sigma_{-;-; 1}^{-;-}(u)\left[\Sigma_{1 ;-; 1}^{1 ;-}(u)-\Sigma_{-; 1 ; 1}^{-; 1}(u)\right] \\
& \Sigma_{2 ;-; 2}^{1 ;-}(u)=\frac{4-u}{4 u} \Sigma_{-;-; 1}^{-;-}(u) \Sigma_{2 ;-; 1}^{1 ;-}(u) \\
& \Sigma_{1 ;-; 2}^{2 ;-}(u)-2 \Sigma_{1 ; 1 ; 2}^{1 ; 1}(u)+\Sigma_{-; 1 ; 2}^{-; 2}(u)+\Sigma_{-; 2 ; 2}^{-; 1}(u)-2 \Sigma_{1 ;-; 3}^{1 ;-}(u)+2 \Sigma_{-; 1 ; 3}^{-; 1}(u)+2 \Sigma_{-;-; 4}^{-;-}(u) \\
& \quad=\frac{4-u}{u}\left[\Sigma_{-;-; 1}^{-;-}(u)\left(\Sigma_{1 ;-; 1}^{2 ;-}(u)-2 \Sigma_{1 ; 1 ; 1}^{1 ; 1}(u)+\Sigma_{-; 1 ; 1}^{-; 2}(u)+\Sigma_{-; 2 ; 1}^{-; 1}(u)-\frac{3}{8} \Sigma_{2 ;-; 1}^{1 ;-}(u)\right)\right. \\
& \left.\quad+\left(\Sigma_{1 ;-; 1}^{1 ;-}(u)-\Sigma_{-; 1 ; 1}^{-; 1}(u)\right)^{2}\right]
\end{aligned}
$$

with $u=4 z$, so that

$$
\frac{4-u}{u}=\frac{1-z}{z}=\cot ^{2} \frac{\theta}{2}
$$

Another interesting relation, Eq. (B.19) of Ref. [16] (which follows from Eq. (20) on p. 498 of [31]), reads

$$
\begin{gathered}
{ }_{3} F_{2}\left(\begin{array}{c}
1+a_{1} \varepsilon, 1+a_{2} \varepsilon, 1+\frac{1}{2}\left(a_{1}+a_{2}\right) \varepsilon \\
\frac{3}{2}+\frac{1}{2}\left(a_{1}+a_{2}\right) \varepsilon, 1+\left(a_{1}+a_{2}\right) \varepsilon
\end{array} \mid z\right)={ }_{2} F_{1}\left(\begin{array}{c}
1+\frac{1}{2} a_{1} \varepsilon, 1+\frac{1}{2} a_{2} \varepsilon \\
\frac{3}{2}+\frac{1}{2}\left(a_{1}+a_{2}\right) \varepsilon
\end{array} \mid z\right) \\
\quad \times\left\{1+\frac{a_{1} a_{2} \varepsilon^{2} z}{2\left[1+\left(a_{1}+a_{2}\right) \varepsilon\right]}{ }_{3} F_{2}\left(\begin{array}{c}
1+\frac{1}{2} a_{1} \varepsilon, 1+\frac{1}{2} a_{2} \varepsilon, 1 \\
\frac{3}{2}+\frac{1}{2}\left(a_{1}+a_{2}\right) \varepsilon, 2
\end{array} \mid z\right)\right\} .
\end{gathered}
$$


In this way, we get the following relations:

$$
\begin{aligned}
& \Sigma_{2 ;-; 1}^{1 ;-}(u)=\frac{1}{3} \Sigma_{-;-; 1}^{-;-}(u) \Sigma_{-;-; 2}^{-;-}(u) \\
& 3 \Sigma_{1,2 ;-; 1}^{1,1 ;-}(u)-3 \Sigma_{2,1 ; 1}^{1 ; 1}(u)-\frac{7}{2} \Sigma_{3 ;-; 1}^{1 ;-}(u) \\
& \quad=\Sigma_{-;-; 1}^{-;-}(u)\left[\Sigma_{1 ;-; 2}^{1 ;-}(u)-\Sigma_{-; 1 ; 2}^{-; 1}(u)\right]+\Sigma_{-;-; 2}^{-;-}(u)\left[\Sigma_{1 ;-; 1}^{1 ;-}(u)-\Sigma_{-; 1 ; 1}^{-; 1}(u)\right]
\end{aligned}
$$

We have checked that all above equations are satisfied by the explicit results for the sums listed in this paper.

Let us also present some relations for higher-order sums:

$$
\begin{aligned}
& \Sigma_{3 ;-; 2}^{1 ;-}(u)+\Sigma_{2 ;-; 3}^{1 ;-}(u)-\Sigma_{2,1 ;-; 2}^{1,1 ;-}(u)+\Sigma_{2 ; 1 ; 2}^{1 ; 1}(u)=\frac{4-u}{4 u}\left\{\Sigma_{2 ;-; 1}^{1 ;-}(u)\left[\Sigma_{-; 1 ; 1}^{-; 1}(u)-\Sigma_{1 ;-; 1}^{1 ;-}(u)\right]\right. \\
& \left.\quad+\Sigma_{-;-; 1}^{-;-}(u)\left[\Sigma_{2 ; 1 ; 1}^{1 ; 1}(u)-\Sigma_{2,1 ;-; 1}^{1,1 ;-}(u)+\frac{1}{2} \Sigma_{3 ;-; 1}^{1 ;-}(u)\right]\right\} \\
& \Sigma_{2 ;-; 2}^{2 ;-}(u)-\Sigma_{4 ;-; 2}^{1 ;-}(u)=\frac{4-u}{16 u}\left\{\left[\Sigma_{2 ;-; 1}^{1 ;-}(u)\right]^{2}+\Sigma_{-;-; 1}^{-;-}(u)\left[\Sigma_{2 ;-; 1}^{2 ;-}(u)-\Sigma_{4 ;-; 1}^{1 ;-}(u)\right]\right\} \\
& \Sigma_{2 ;-; 1}^{2 ;-}(u)-\Sigma_{4 ;-; 1}^{1 ;-}(u)=\frac{2}{45} \Sigma_{-;-; 1}^{-;-}(u)\left\{\left[\Sigma_{-;-; 2}^{-;-}(u)\right]^{2}+3 \Sigma_{2 ;-; 2}^{1 ;-}(u)\right\} \\
& 2\left[\Sigma_{-;-; 2}^{-;-}(u)\right]^{2}\left[\Sigma_{1 ;-; 1}^{1 ;-}(u)-\Sigma_{-; 1 ; 1}^{-; 1}(u)\right]+4 \Sigma_{-;-; 2}^{-;-}(u) \Sigma_{-;-; 1}^{-;-}(u)\left[\Sigma_{1 ;-; 2}^{1 ;-}(u)-\Sigma_{-; 1 ; 2}^{-; ;}(u)\right] \\
& +6 \Sigma_{2 ;-; 2}^{1 ;-}(u)\left[\Sigma_{1 ;-; 1}^{1 ;-}(u)-\Sigma_{-; 1 ; 1}^{-; 1}(u)\right]+6 \Sigma_{-;-; 1}^{-;-}(u)\left[\Sigma_{2,1 ;-; 2}^{1,1 ;-}(u)-\Sigma_{2 ; 1 ;-}^{1 ; 1}(u)\right] \\
& +45 \Sigma_{2 ; 1 ; 1}^{2 ; 1}(u)-45 \Sigma_{2,1 ;-; 1}^{2 ; 1 ;-}(u)+45 \Sigma_{4,1 ;-; 1}^{1,1 ;-}(u)-45 \Sigma_{4 ; 1 ; 1}^{1 ; 1}(u)+93 \Sigma_{3,2 ;-; 1}^{1 ; 1 ;-}(u)-93 \Sigma_{5 ;-; 1}^{1 ;-}(u) \\
& +4 \Sigma_{3 ;-; 1}^{1 ;-}(u) \Sigma_{-;-; 2}^{-;-}(u)-3 \Sigma_{3 ;-; 2}^{1 ;-}(u) \Sigma_{-;-; 1}^{-;-}(u)=0
\end{aligned}
$$

where Eqs. (B.8) and (B.9) follow from Eq. (B.1), while Eqs. (B.9) and (B.10) follow from Eq. (B.6).

One more relation can be derived from Eq. (47) on p. 456 of [31],

$$
{ }_{2} F_{1}\left(\begin{array}{c}
1+a_{1} \varepsilon, 1+a_{2} \varepsilon \\
\frac{3}{2}+\frac{1}{2}\left(a_{1}+a_{2}\right) \varepsilon
\end{array} \mid \sin ^{2} \frac{\theta}{2}\right)=\cos \theta{ }_{2} F_{1}\left(\begin{array}{c}
1+\frac{1}{2} a_{1} \varepsilon, 1+\frac{1}{2} a_{2} \varepsilon \\
\frac{3}{2}+\frac{1}{2}\left(a_{1}+a_{2}\right) \varepsilon
\end{array} \mid \sin ^{2} \theta\right) .
$$

We can compare the $\varepsilon$-expansions of these ${ }_{2} F_{1}$ functions, considering the coefficients of $\varepsilon^{k}$ as functions of $\theta$. Introducing

$$
u=4 \sin ^{2} \frac{\theta}{2}, \quad \widetilde{u}=4 \sin ^{2} \theta, \quad A_{1}=a_{1}+a_{2}, \quad A_{2}=a_{1}^{2}+a_{2}^{2},
$$

we get the following relations at orders $\varepsilon^{0}$ and $\varepsilon^{1}$ :

$$
\begin{aligned}
\cot \frac{\theta}{2} \sum_{j=1}^{\infty} \frac{1}{\left(\begin{array}{c}
2 j \\
j
\end{array}\right)} \frac{u^{j}}{j} & =\frac{1}{2} \cot \theta \sum_{j=1}^{\infty} \frac{1}{\left(\begin{array}{c}
2 j \\
j
\end{array}\right)} \frac{\widetilde{u}^{j}}{j} \\
\cot \frac{\theta}{2} \sum_{j=1}^{\infty} \frac{1}{\left(\begin{array}{c}
2 j \\
j
\end{array}\right)} \frac{u^{j}}{j}\left(\frac{3}{2} S_{1}-\bar{S}_{1}\right) & =\frac{1}{2} \cot \theta \sum_{j=1}^{\infty} \frac{1}{\left(\begin{array}{c}
2 j \\
j
\end{array}\right)} \frac{\widetilde{u}^{j}}{j}\left(S_{1}-\bar{S}_{1}\right) .
\end{aligned}
$$


At order $\varepsilon^{2}$, comparison of the coefficients of $A_{2}$ and $A_{1}^{2}$ yields

$$
\begin{gathered}
\cot \frac{\theta}{2} \sum_{j=1}^{\infty} \frac{1}{\left(\begin{array}{c}
2 j \\
j
\end{array}\right)} \frac{u^{j}}{j} S_{2}=\frac{1}{8} \cot \theta \sum_{j=1}^{\infty} \frac{1}{\left(\begin{array}{c}
2 j \\
j
\end{array}\right)} \frac{\widetilde{u}^{j}}{j} S_{2}, \\
\cot \frac{\theta}{2} \sum_{j=1}^{\infty} \frac{1}{\left(\begin{array}{c}
2 j \\
j
\end{array}\right)} \frac{u^{j}}{j}\left[\frac{1}{2}\left(\bar{S}_{2}+\bar{S}_{1}^{2}\right)-\frac{3}{2} S_{1} \bar{S}_{1}-\frac{1}{8} S_{2}+\frac{9}{8} S_{1}^{2}\right] \\
=\frac{1}{2} \cot \theta \sum_{j=1}^{\infty} \frac{1}{\left(\begin{array}{c}
2 j \\
j
\end{array}\right)} \frac{\widetilde{u}^{j}}{j}\left[\frac{1}{2}\left(\bar{S}_{2}+\bar{S}_{1}^{2}\right)-S_{1} \bar{S}_{1}-\frac{1}{8} S_{2}+\frac{1}{2} S_{1}^{2}\right] .
\end{gathered}
$$

All these equations are satisfied by analytic expressions for these sums in terms of $\theta$ from Section 2 of this paper. Remember that one needs to substitute $\theta \rightarrow 2 \theta$ as an argument on the r.h.s.

At order $\varepsilon^{3}$, we have two independent structures, $A_{1}^{3}$ and $A_{1} A_{2}$. It is more convenient, however, to compare the coefficients of $A_{1}^{3}$ and $A_{1}\left(A_{1}^{2}-A_{2}\right)$. The first equation coming from the coefficients of $A_{1}^{3}$ yields

$$
\cot \frac{\theta}{2} \sum_{j=1}^{\infty} \frac{1}{\left(\begin{array}{c}
2 j \\
j
\end{array}\right)} \frac{u^{j}}{j}\left(\frac{3}{16} \mathcal{C}_{0}+\frac{3}{4} \mathcal{C}_{1}+\frac{1}{2} \mathcal{C}_{2}\right)=\frac{1}{2} \cot \theta \sum_{j=1}^{\infty} \frac{1}{\left(\begin{array}{c}
2 j \\
j
\end{array}\right)} \frac{\widetilde{u}^{j}}{j}\left(\frac{1}{24} \mathcal{C}_{0}+\frac{1}{2} \mathcal{C}_{1}+\frac{1}{2} \mathcal{C}_{2}\right),
$$

where $\mathcal{C}_{j}$ are the combinations of the harmonic sums defined in Eqs. (2.5). This equation is also satisfied, if we use analytic expressions for these sums in terms of $\theta$ given in Section 2.

The second equation at order $\varepsilon^{3}$ comes from the coefficient of $A_{1}\left(A_{1}^{2}-A_{2}\right)$,

$$
\cot \frac{\theta}{2} \sum_{j=1}^{\infty} \frac{1}{\left(\begin{array}{c}
2 j \\
j
\end{array}\right)} \frac{u^{j}}{j}\left(-\frac{3}{4} S_{1} S_{2}+\frac{1}{2} S_{2} \bar{S}_{1}+\frac{1}{2} S_{3}\right)=\frac{1}{2} \cot \theta \sum_{j=1}^{\infty} \frac{1}{\left(\begin{array}{c}
2 j \\
j
\end{array}\right)} \frac{\widetilde{u}^{j}}{j}\left(-\frac{1}{8} S_{1} S_{2}+\frac{1}{8} S_{2} \bar{S}_{1}+\frac{1}{16} S_{3}\right) .
$$

If we introduce two functions

$$
\begin{aligned}
& \Psi_{1}(\theta)=\cot \frac{\theta}{2} \sum_{j=1}^{\infty} \frac{1}{\left(\begin{array}{c}
2 j \\
j
\end{array}\right)} \frac{u^{j}}{j}\left(S_{1} S_{2}-S_{3}\right), \\
& \Psi_{2}(\theta)=\cot \frac{\theta}{2} \sum_{j=1}^{\infty} \frac{1}{\left(\begin{array}{c}
2 j \\
j
\end{array}\right)} \frac{u^{j}}{j}\left(S_{3}-2 S_{2} \bar{S}_{1}\right),
\end{aligned}
$$

we obtain an interesting relation between them,

$$
3 \Psi_{1}(\theta)+\Psi_{2}(\theta)=\frac{1}{4} \Psi_{1}(2 \theta)+\frac{1}{8} \Psi_{2}(2 \theta) .
$$

This relation is satisfied by the explicit results for the sums involved given in Eqs. (2.67), (2.68), (2.80).

\section{Further results for the inverse binomial sums}

For completeness, in this Appendix we collect some results for the multiple inverse binomial sums of lower weights. They can be obtained by applying the operator $u(\mathrm{~d} / \mathrm{d} u)$ to our 
results presented in sections 2 and 3. These sums occur in lower terms of the $\varepsilon$-expansion of hypergeometric functions given in Eq. (2.30).

First of all, we list explicit results for some particular cases of the general formulae (1.2) and (2.33), in terms of the angular variable $\theta$ (1.3):

$$
\begin{aligned}
\sum_{j=1}^{\infty} \frac{1}{\left(\begin{array}{c}
2 j \\
j
\end{array}\right)} \frac{u^{j}}{j} & =\theta \tan \frac{\theta}{2} \\
\sum_{j=1}^{\infty} \frac{1}{\left(\begin{array}{c}
2 j \\
j
\end{array}\right)} \frac{u^{j}}{j^{2}} & =\frac{1}{2} \theta^{2} \\
\sum_{j=1}^{\infty} \frac{1}{\left(\begin{array}{c}
2 j \\
j
\end{array}\right)} \frac{u^{j}}{j^{4}} & =-2 \mathrm{Ls}_{4}^{(1)}(\theta)+4 l_{\theta}\left[\mathrm{Cl}_{3}(\theta)+\theta \mathrm{Cl}_{2}(\theta)-\zeta_{3}\right]+\theta^{2} l_{\theta}^{2} \\
\sum_{j=1}^{\infty} \frac{1}{\left(\begin{array}{c}
2 j \\
j
\end{array}\right)} \frac{u^{j}}{j^{2}} S_{2} & =\frac{1}{24} \theta^{4} .
\end{aligned}
$$

Then, let us present a few more complicated examples of the results in terms of $\theta$,

$$
\begin{aligned}
\sum_{j=1}^{\infty} \frac{1}{\left(\begin{array}{c}
2 j \\
j
\end{array}\right)} & \frac{u^{j}}{j} S_{1}^{2} \bar{S}_{1}=\tan \frac{\theta}{2}\left\{\mathrm{Cl}_{2}(\pi-\theta)\left[28 L_{\theta}^{2}-8 L_{\theta} l_{\theta}+\frac{5}{2} \theta^{2}\right]+\mathrm{Cl}_{2}(2 \theta)\left[2 L_{\theta}^{2}+\frac{1}{4} \theta^{2}\right]\right. \\
& +4 L_{\theta} \mathrm{Ls}_{3}(\theta)-2 L_{\theta} \mathrm{Ls}_{3}(2 \theta)+4 \mathrm{Lsc}_{2,3}(\theta)-\frac{1}{2} \theta^{2} \mathrm{Cl}_{2}(\theta)-\mathrm{Cl}_{4}(\theta) \\
& +\left[\mathrm{Ls}_{3}(\pi-\theta)-\mathrm{Ls}_{3}(\pi)\right]\left[4 l_{\theta}-28 L_{\theta}\right]-8 \theta\left[\mathrm{Cl}_{3}(\pi-\theta)-\mathrm{Cl}_{3}(\pi)\right] \\
& +8\left[\mathrm{Ls}_{4}(\pi-\theta)-\mathrm{Ls}_{4}(\pi)\right]-16\left[\mathrm{Cl}_{4}(\pi-\theta)-\mathrm{Cl}_{4}(\pi)\right] \\
& \left.+\frac{1}{6} \theta^{3} l_{\theta}-\frac{2}{3} \theta^{3} L_{\theta}-8 \theta L_{\theta}^{3}+13 \zeta_{3} \theta+4 \theta l_{\theta} L_{\theta}^{2}\right\} \\
\sum_{j=1}^{\infty} \frac{1}{\left(\begin{array}{c}
2 j \\
j
\end{array}\right)} & \frac{u^{j}}{j} S_{1}\left[\bar{S}_{1}^{2}+\bar{S}_{2}\right]=\tan \frac{\theta}{2}\left\{\mathrm{Cl}_{2}(\pi-\theta)\left[2 \theta^{2}+2 l_{\theta}^{2}-20 L_{\theta} l_{\theta}+32 L_{\theta}^{2}\right]-\frac{1}{3} \mathrm{Ls}_{4}(2 \theta)\right. \\
& +\mathrm{Cl}_{2}(2 \theta)\left[\frac{1}{4} \theta^{2}-2 L_{\theta} l_{\theta}+4 L_{\theta}^{2}\right]+\mathrm{Ls}_{3}(2 \theta)\left[l_{\theta}-4 L_{\theta}\right]-2 \mathrm{Ls}_{3}(\theta)\left[l_{\theta}-5 L_{\theta}\right] \\
& +\left[\mathrm{Ls}_{3}(\pi-\theta)-\mathrm{Ls}_{3}(\pi)\right]\left[10 l_{\theta}-32 L_{\theta}\right]-6 \theta\left[\mathrm{Cl}_{3}(\pi-\theta)-\mathrm{Cl}_{3}(\pi)\right]-\mathrm{Cl}_{4}(\theta) \\
& +\frac{22}{3}\left[\mathrm{Ls}_{4}(\pi-\theta)-\mathrm{Ls}_{4}(\pi)\right]+10 \mathrm{Lsc}_{2,3}(\theta)-12 \mathrm{Cl}_{4}(\pi-\theta)+\frac{2}{3} \mathrm{Ls}_{4}(\theta) \\
& \left.-\frac{1}{2} \theta^{2} \mathrm{Cl}_{2}(\theta)+10 \zeta_{3} \theta+\frac{1}{6} \theta^{3} l_{\theta}-\frac{1}{2} \theta^{3} L_{\theta}-8 \theta L_{\theta}^{3}+8 \theta L_{\theta}^{2} l_{\theta}-2 \theta L_{\theta} l_{\theta}^{2}\right\} \\
& \left.+12 \mathrm{Cl}_{4}(\pi-\theta)+\frac{1}{4} \theta^{3} l_{\theta}-\frac{1}{2} \theta^{3} L_{\theta}+8 \theta l_{\theta}^{3}-6 \theta l_{\theta}^{2} L_{\theta}+12 \theta l_{\theta} L_{\theta}^{2}-8 \theta L_{\theta}^{3}-7 \theta l_{\theta}^{3}\right\} \\
& +\frac{3}{2} \mathrm{Cl}_{2}(2 \theta)\left[\frac{1}{4} \theta^{2}+\left(2 L_{\theta}-l_{\theta}\right)^{2}\right]+18 \mathrm{Lsc}_{2,3}(\theta)-\frac{3}{2} \mathrm{Cl}_{4}(\theta)-\frac{3}{4} \theta^{2} \mathrm{Cl}_{2}(\theta) \\
& -18\left(2 L_{\theta}-l_{\theta}\right)\left[\mathrm{Ls}_{3}(\pi-\theta)-\mathrm{Ls}_{3}(\pi)-\frac{1}{2} \mathrm{Ls}_{3}(\theta)+\frac{1}{6} \mathrm{Ls}_{3}(2 \theta)\right]+\frac{21}{2} \zeta_{3} \theta \\
\sum_{j=1}^{\infty} \frac{1}{\left(\begin{array}{c}
2 j \\
j
\end{array}\right)} & \frac{u^{j}}{j}\left[\bar{S}_{1}^{3}+3 \bar{S}_{1} \bar{S}_{2}+2 \bar{S}_{3}\right]=\tan \frac{\theta}{2}\left\{9 \mathrm{Cl}_{2}(\pi-\theta)\left[\frac{1}{4} \theta^{2}+\left(2 L_{\theta}-l_{\theta}\right)^{2}\right]\right. \\
& \left.+6)-\mathrm{Ls}_{4}(\pi)\right]-6 \theta\left[\mathrm{Cl}_{3}(\pi-\theta)-\mathrm{Cl}_{3}(\pi)\right]-\mathrm{Ls}_{4}(2 \theta)+3 \mathrm{Ls}_{4}(\theta) \\
& \\
&
\end{aligned}
$$


Using the analytic continuation procedure described in Section 3, these results can be rewritten in terms of the conformal variable $y$ defined in Eq. (3.2). Below we list these analytically-continued results for Eqs. (C.1)-(C.4), as well as for those sums from Section 2 whose analytical continuations were not presented in Section 3:

$$
\begin{aligned}
& \sum_{j=1}^{\infty} \frac{1}{\left(\begin{array}{c}
2 j \\
j
\end{array}\right)} \frac{u^{j}}{j}=\frac{1-y}{1+y} \ln y \\
& \sum_{j=1}^{\infty} \frac{1}{\left(\begin{array}{c}
2 j \\
j
\end{array}\right)} \frac{u^{j}}{j^{2}}=-\frac{1}{2} \ln ^{2} y \\
& \sum_{j=1}^{\infty} \frac{1}{\left(\begin{array}{c}
2 j \\
j
\end{array}\right)} \frac{u^{j}}{j^{3}}=2 \operatorname{Li}_{3}(y)-2 \ln y \operatorname{Li}_{2}(y)-\ln ^{2} y \ln (1-y)+\frac{1}{6} \ln ^{3} y-2 \zeta_{3}, \\
& \sum_{j=1}^{\infty} \frac{1}{\left(\begin{array}{c}
2 j \\
j
\end{array}\right)} \frac{u^{j}}{j^{4}}=4 \mathrm{~S}_{2,2}(y)-4 \mathrm{Li}_{4}(y)-4 \mathrm{~S}_{1,2}(y) \ln y+4 \mathrm{Li}_{3}(y) \ln (1-y)+2 \operatorname{Li}_{3}(y) \ln y \\
& -4 \operatorname{Li}_{2}(y) \ln y \ln (1-y)-\ln ^{2} y \ln ^{2}(1-y)+\frac{1}{3} \ln ^{3} y \ln (1-y)-\frac{1}{24} \ln ^{4} y \\
& -4 \ln (1-y) \zeta_{3}+2 \ln y \zeta_{3}+3 \zeta_{4} \text {, } \\
& \sum_{j=1}^{\infty} \frac{1}{\left(\begin{array}{c}
2 j \\
j
\end{array}\right)} \frac{u^{j}}{j} S_{1}=\frac{1-y}{1+y}\left[-2 \operatorname{Li}_{2}(-y)-2 \ln y \ln (1+y)+\frac{1}{2} \ln ^{2} y-\zeta_{2}\right], \\
& \sum_{j=1}^{\infty} \frac{1}{\left(\begin{array}{c}
2 j \\
j
\end{array}\right)} \frac{u^{j}}{j} \bar{S}_{1}=\frac{1-y}{1+y}\left[\operatorname{Li}_{2}(y)-2 \operatorname{Li}_{2}(-y)-2 \ln y \ln (1+y)+\ln y \ln (1-y)\right. \\
& \left.+\frac{1}{4} \ln ^{2} y-2 \zeta_{2}\right] \text {, } \\
& \sum_{j=1}^{\infty} \frac{1}{\left(\begin{array}{c}
2 j \\
j
\end{array}\right)} \frac{u^{j}}{j} S_{1}^{2}=\frac{1-y}{1+y}\left[8 \mathrm{~S}_{1,2}(-y)-4 \mathrm{Li}_{3}(-y)+8 \mathrm{Li}_{2}(-y) \ln (1+y)+4 \ln ^{2}(1+y) \ln y\right. \\
& \left.-2 \ln (1+y) \ln ^{2} y+\frac{1}{6} \ln ^{3} y+4 \zeta_{2} \ln (1+y)-2 \zeta_{2} \ln y-4 \zeta_{3}\right], \\
& \sum_{j=1}^{\infty} \frac{1}{\left(\begin{array}{c}
2 j \\
j
\end{array}\right)} \frac{u^{j}}{j} S_{1} \bar{S}_{1}=\frac{1-y}{1+y}\left[10 \mathrm{~S}_{1,2}(-y)-\mathrm{S}_{1,2}\left(y^{2}\right)+\mathrm{Li}_{3}(y)+2 \mathrm{~S}_{1,2}(y)-3 \mathrm{Li}_{3}(-y)\right. \\
& -2 \ln (1-y) \mathrm{Li}_{2}(-y)+8 \ln (1+y) \mathrm{Li}_{2}(-y)-2 \ln (1+y) \mathrm{Li}_{2}(y) \\
& -2 \ln y \ln (1-y) \ln (1+y)-\ln y \ln ^{2}(1-y)+4 \ln y \ln ^{2}(1+y) \\
& +\frac{1}{2} \ln ^{2} y \ln (1-y)-\frac{3}{2} \ln ^{2} y \ln (1+y)+\frac{1}{12} \ln ^{3} y+6 \zeta_{2} \ln (1+y) \\
& \left.-\zeta_{2} \ln (1-y)-\frac{5}{2} \zeta_{2} \ln y-\frac{11}{2} \zeta_{3}\right] \text {, } \\
& \sum_{j=1}^{\infty} \frac{1}{\left(\begin{array}{c}
2 j \\
j
\end{array}\right)} \frac{u^{j}}{j} S_{2}=-\frac{1-y}{6(1+y)} \ln ^{3} y \\
& \sum_{j=1}^{\infty} \frac{1}{\left(\begin{array}{c}
2 j \\
j
\end{array}\right)} \frac{u^{j}}{j^{2}} S_{2}=\frac{1}{24} \ln ^{4} y
\end{aligned}
$$




$$
\begin{aligned}
\sum_{j=1}^{\infty} \frac{1}{\left(\begin{array}{c}
2 j \\
j
\end{array}\right)} \frac{u^{j}}{j} S_{3}= & \frac{1-y}{1+y}\left[\frac{1}{24} \ln ^{4} y+6 \operatorname{Li}_{4}(y)+\ln ^{2} y \operatorname{Li}_{2}(y)-2 \zeta_{3} \ln y-4 \ln y \operatorname{Li}_{3}(y)-6 \zeta_{4}\right], \\
\sum_{j=1}^{\infty} \frac{1}{\left(\begin{array}{c}
2 j \\
j
\end{array}\right)} \frac{u^{j}}{j} S_{1} S_{2}= & \frac{1-y}{1+y}\left[\frac{1}{3} \ln ^{3} y \ln (1+y)-\frac{1}{24} \ln ^{4} y+\frac{1}{2} \zeta_{2} \ln ^{2} y+\ln ^{2} y \operatorname{Li}_{2}(-y)+\ln ^{2} y \operatorname{Li}_{2}(y)\right. \\
& \left.+\zeta_{3} \ln y-4 \ln y \operatorname{Li}_{3}(-y)-4 \ln y \operatorname{Li}_{3}(y)+\zeta_{4}+8 \operatorname{Li}_{4}(-y)+6 \operatorname{Li}_{4}(y)\right],(\mathrm{C} .19) \\
\sum_{j=1}^{\infty} \frac{1}{\left(\begin{array}{c}
2 j \\
j
\end{array}\right)} \frac{u^{j}}{j} S_{2} \bar{S}_{1}= & \frac{1-y}{1+y}\left[\frac{1}{3} \ln ^{3} y \ln (1+y)-\frac{1}{6} \ln ^{3} y \ln (1-y)-\frac{1}{48} \ln ^{4} y+\zeta_{2} \ln ^{2} y+4 \zeta_{3} \ln y\right. \\
& \left.+\ln ^{2} y \operatorname{Li}_{2}(-y)-4 \ln y \operatorname{Li}_{3}(-y)+8 \zeta_{4}+8 \operatorname{Li}_{4}(-y)-\operatorname{Li}_{4}(y)\right] . \quad(\mathrm{C} .20)
\end{aligned}
$$

For the combination (2.23) involving $\left(\bar{S}_{2}+\bar{S}_{1}^{2}\right)$ we get

$$
\begin{aligned}
& \sum_{j=1}^{\infty} \frac{1}{\left(\begin{array}{c}
2 j \\
j
\end{array}\right)} \frac{u^{j}}{j}\left(\bar{S}_{2}+\bar{S}_{1}^{2}\right)=\frac{1-y}{1+y}\left\{12 \mathrm{~S}_{1,2}(-y)-2 \mathrm{~S}_{1,2}\left(y^{2}\right)+\operatorname{Li}_{3}(y)+6 \mathrm{~S}_{1,2}(y)-8 \zeta_{3}\right. \\
& \quad-2 \operatorname{Li}_{3}(-y)-4 \ln \left(1-y^{2}\right) \operatorname{Li}_{2}(y)+2 \ln (1-y) \operatorname{Li}_{2}(-y)+8 \ln (1+y) \operatorname{Li}_{2}(-y)-2 \zeta_{2} \ln y \\
& \left.\left.\quad+\ln y[\ln (1-y)-2 \ln (1+y)]^{2}+\left(\frac{1}{2} \ln ^{2} y-4 \zeta_{2}\right)[\ln (1-y)-2 \ln (1+y)]\right\}, \quad \text { (C. } 21\right)
\end{aligned}
$$

For a separate term of this sum involving $\bar{S}_{2}$, we get

$$
\sum_{j=1}^{\infty} \frac{1}{\left(\begin{array}{c}
2 j \\
j
\end{array}\right)} \frac{u^{j}}{j} \bar{S}_{2}=\frac{1-y}{1+y}\left[2 \operatorname{Li}_{3}\left(-\omega_{s}\right)-2 \operatorname{Li}_{3}\left(\omega_{s}\right)-\frac{1}{24} \ln ^{3} y\right]
$$

where $\omega_{s}$ is defined in Eq. (3.6).

The results for the multiple inverse binomial sums presented in this paper are summarized in Table 1. When two equation numbers are present, the first one refers to the result in terms of the angular variable $\theta$, whereas the second one corresponds to its analytical continuation. An asterisk means that the corresponding equation holds for general $c$. The symbol $\dagger$ means that the results for the sums involving the combinations $S_{1}\left(\bar{S}_{2}+\bar{S}_{1}^{2}\right)$ and $\bar{S}_{1}^{3}+3 \bar{S}_{1} \bar{S}_{2}+2 \bar{S}_{3}$ can be extracted from the expressions (3.10) and (3.11) given for the sums involving $\mathcal{C}_{1}$ and $\mathcal{C}_{2}$, respectively, using the definitions (2.4).

\section{Connection between binomial, harmonic and inverse binomial sums}

Using the procedure described in section 2.2, one can also construct the $\varepsilon$-expansion of hypergeometric functions of the following types:

$$
{ }_{P+1} F_{P}\left(\begin{array}{c}
\frac{3}{2}+b_{1} \varepsilon, \ldots, \frac{3}{2}+b_{J} \varepsilon, 1+a_{1} \varepsilon, \ldots, 1+a_{K} \varepsilon, 2+d_{1} \varepsilon, \ldots, 2+d_{L} \varepsilon \\
\frac{3}{2}+f_{1} \varepsilon, \ldots, \frac{3}{2}+f_{J-1} \varepsilon, 1+e_{1} \varepsilon, \ldots, 1+e_{R} \varepsilon, 2+c_{1} \varepsilon, \ldots, 2+c_{K+L-R} \varepsilon
\end{array} \mid u\right)
$$




\begin{tabular}{|c|c|c|c|c|}
\hline & $c=1$ & $c=2$ & $c=3$ & $c=4$ \\
\hline 1 & (C.1), (C.8 & (C.2), (C.9) & $(2.66),(\mathrm{C} .10)$ & (C.3), (C.11) \\
\hline$S_{1}$ & $(2.19)$, C.12 & $(2.35,(3.21$ & $(2.43),(3.12$ & \\
\hline $\bar{S}_{1}$ & (2.20), (C.13 & $(2.36),(3.22)$ & (2.44), (3.13) & \\
\hline$S_{2}$ & (2.8), (C.16) & (C.4), C.17 & $(2.33)^{\star}$ & $(2.33)^{\star}$ \\
\hline$S_{1}^{2}$ & (2.21), (C.14 & $(2.37),(3.7)$ & & \\
\hline$S_{1} \bar{S}_{1}$ & $(2.22)$, C.15 & $(2.38),(3.8)$ & & \\
\hline $\bar{S}_{2}$ & (2.88), (C.22) & $(2.89),(3.19)$ & & \\
\hline $\bar{S}_{2}+\bar{S}_{1}^{2}$ & (2.23), (C.21) & (2.39), (3.9) & & \\
\hline$S_{3}$ & (2.67), (C.18) & (2.81), (3.15) & & \\
\hline$S_{1} S_{2}$ & (2.68), (C.19) & $(2.82),(3.16)$ & & \\
\hline$S_{1}^{3}$ & (2.69), (3.14) & & & \\
\hline$S_{2} \bar{S}_{1}$ & (2.80), (C.20) & $(2.83),(3.17$ & & \\
\hline$S_{1}^{2} \bar{S}_{1}$ & (C.5), 3.18 & & & \\
\hline$S_{1}\left(\bar{S}_{2}+\bar{S}_{1}^{2}\right)$ & $(\mathrm{C.6}),(3.10)^{\dagger}$ & & & \\
\hline $\begin{array}{c}\bar{S}_{1}^{3}+3 \bar{S}_{1} \bar{S}_{2}+2 \bar{S}_{3} \\
S_{2}^{2}-S_{4}\end{array}$ & $\frac{(\text { C.7) }}{(3.11)^{\dagger}}$ & $(2.34)^{\star}$ & $(234)^{\star}$ & $(234) *$ \\
\hline & & & & \\
\hline
\end{tabular}

Table 1: Equation index for the inverse binomial sums

$$
{ }_{P+1} F_{P}\left(\begin{array}{c}
\frac{3}{2}+b_{1} \varepsilon, \ldots, \frac{3}{2}+b_{J} \varepsilon, 1+a_{1} \varepsilon, \ldots, 1+a_{K} \varepsilon, 2+d_{1} \varepsilon, \ldots, 2+d_{L} \varepsilon \\
\frac{3}{2}+f_{1} \varepsilon, \ldots, \frac{3}{2}+f_{J} \varepsilon, 1+e_{1} \varepsilon, \ldots, 1+e_{R} \varepsilon, 2+c_{1} \varepsilon, \ldots, 2+c_{K+L-R-1} \varepsilon
\end{array} \mid z\right),
$$

where $P=K+L+J-1$ and $u=4 z$. By analogy with Eq. (2.29), the $\varepsilon$-expansion of these functions can be written in the following form:

$$
\begin{aligned}
& P_{P+1} F_{P}\left(\begin{array}{c}
\left\{\frac{3}{2}+b_{i} \varepsilon\right\}^{J},\left\{1+a_{i} \varepsilon\right\}^{K},\left\{2+d_{i} \varepsilon\right\}^{L} \\
\left\{\frac{3}{2}+f_{i} \varepsilon\right\}^{J-1},\left\{1+e_{i} \varepsilon\right\}^{R},\left\{2+c_{i} \varepsilon\right\}^{K+L-R}
\end{array} \mid u\right) \\
&= \frac{2}{u} \frac{\prod_{s=1}^{K+L-R}\left(1+c_{s} \varepsilon\right) \prod_{k=1}^{J-1}\left(1+2 f_{k} \varepsilon\right)}{\prod_{i=1}^{L}\left(1+d_{i} \varepsilon\right) \Pi_{r=1}^{J}\left(1+2 b_{r} \varepsilon\right)} \sum_{j=1}^{\infty}\left(\begin{array}{c}
2 j \\
j
\end{array}\right) \frac{z^{j}}{j^{K-R-1}} \Delta, \\
&{ }_{P+1} F_{P}\left(\begin{array}{c}
\left\{\frac{3}{2}+b_{i} \varepsilon\right\}^{J},\left\{1+a_{i} \varepsilon\right\}^{K},\left\{2+d_{i} \varepsilon\right\}^{L} \\
\left\{\frac{3}{2}+f_{i} \varepsilon\right\}^{J},\left\{1+e_{i} \varepsilon\right\}^{R},\left\{2+c_{i} \varepsilon\right\}^{K+L-R-1}
\end{array} \mid z\right) \\
&=\frac{1}{z} \frac{\prod_{s=1}^{K+L-R-1}\left(1+c_{s} \varepsilon\right) \prod_{k=1}^{J}\left(1+2 f_{k} \varepsilon\right)}{\prod_{i=1}^{L}\left(1+d_{i} \varepsilon\right) \prod_{r=1}^{J}\left(1+2 b_{r} \varepsilon\right)} \sum_{j=1}^{\infty} \frac{z^{j}}{j^{K-R-1}} \Delta,
\end{aligned}
$$

where the function $\Delta$ is defined in the same way as in Eq. (2.30). One should only remember that the upper summation limits for the coefficients $B_{k}$ and $C_{k}$ are changed, since the numbers of the parameters $b_{i}$ and $c_{i}$ in Eqs. (D.1) and (D.2) are different.

The sums appearing in Eq. (D.1) are expressible in terms of the multiple binomial sums [8], whereas the sums of Eq. (D.2) are reduced to the multiple harmonic sums. Using relations between hypergeometric function of different arguments, it is possible to express one type 
of sums in terms of the another one, plus some trivial part. Let us illustrate this on the example of ${ }_{2} F_{1}$ function (2.1). Using the standard formula of analytic continuation to the argument $1 / z$, we obtain a combination of two ${ }_{2} F_{1}$ functions. To bring them to the form of (D.1), we need to shift some of the parameters using

$$
{ }_{2} F_{1}\left(\begin{array}{c|c}
a, b & z \\
c & z
\end{array}\right)=1+\frac{a b z}{c}{ }_{3} F_{2}\left(\begin{array}{c|c}
1, a+1, b+1 \\
2, c+1
\end{array} \mid z\right) .
$$

Finally, using the duplication formula for the argument of the $\Gamma$-function, we arrive at the following relation:

$$
\begin{aligned}
& 2 z\left(a_{1}-a_{2}\right) \varepsilon \frac{\Gamma(1+b \varepsilon)}{\Gamma(2+2 b \varepsilon)}{ }_{2} F_{1}\left(\begin{array}{c}
1+a_{1} \varepsilon, 1+a_{2} \varepsilon \\
\frac{3}{2}+b \varepsilon
\end{array} \mid z\right) \\
& =\frac{1}{(-4 z)^{a_{1} \varepsilon}} \frac{\Gamma\left(1+\left(a_{2}-a_{1}\right) \varepsilon\right) \Gamma\left(1+\left(b-a_{1}\right) \varepsilon\right)}{\Gamma\left(1+a_{2} \varepsilon\right) \Gamma\left(1+2\left(b-a_{1}\right) \varepsilon\right)} \\
& \times\left\{1+\frac{\left(1+a_{1} \varepsilon\right)\left(1+2\left(a_{1}-b\right) \varepsilon\right)}{2 z\left(1+\left(a_{1}-a_{2}\right) \varepsilon\right)}{ }_{3} F_{2}\left(\begin{array}{c|c}
1,2+a_{1} \varepsilon, \frac{3}{2}+\left(a_{1}-b\right) \varepsilon & \frac{1}{z} \\
2,2+\left(a_{1}-a_{2}\right) \varepsilon & 2
\end{array}\right)\right\} \\
& -\frac{1}{(-4 z)^{a_{2} \varepsilon}} \frac{\Gamma\left(1+\left(a_{1}-a_{2}\right) \varepsilon\right) \Gamma\left(1+\left(b-a_{2}\right) \varepsilon\right)}{\Gamma\left(1+a_{1} \varepsilon\right) \Gamma\left(1+2\left(b-a_{2}\right) \varepsilon\right)} \\
& \times\left\{1+\frac{\left(1+a_{2} \varepsilon\right)\left(1+2\left(a_{2}-b\right) \varepsilon\right)}{2 z\left(1+\left(a_{2}-a_{1}\right) \varepsilon\right)}{ }_{3} F_{2}\left(\begin{array}{c|c}
1,2+a_{2} \varepsilon, \frac{3}{2}+\left(a_{2}-b\right) \varepsilon & \frac{1}{z} \\
2,2+\left(a_{2}-a_{1}\right) \varepsilon & z
\end{array}\right\} .\right.
\end{aligned}
$$

Here, the $\varepsilon$-expansion of the hypergeometric function on the l.h.s. can be expressed in terms of the multiple inverse binomial sums (see Eq. (2.29)), whereas the functions on the r.h.s. yield the multiple binomial sums (see Eq. (D.1)).

Furthermore, the following three quadratic relations for ${ }_{2} F_{1}$ functions (see, e.g., in [31]) allow us to connect some multiple binomial sums (appearing in Eq. (D.1) ) with multiple harmonic sums (see Eq. (D.2)):

$$
\begin{aligned}
& { }_{2} F_{1}\left(\begin{array}{c}
1+b \varepsilon, \frac{3}{2}+a \varepsilon \\
2+(a+b) \varepsilon
\end{array} \mid u\right)=\frac{1}{\sqrt{1-u}}(1+\chi)^{2+2 a \varepsilon}{ }_{2} F_{1}\left(\begin{array}{c}
2+2 a \varepsilon, 1+(a-b) \varepsilon \\
2+(a+b) \varepsilon
\end{array} \mid-\chi\right), \quad \text { (D.4) } \\
& { }_{2} F_{1}\left(\begin{array}{c}
1+b \varepsilon, \frac{3}{2}+a \varepsilon \\
2+2 b \varepsilon
\end{array} \mid u\right)=(1+\chi)^{3+2 a \varepsilon}{ }_{2} F_{1}\left(\begin{array}{c}
\frac{3}{2}+a \varepsilon, 1+(a-b) \varepsilon \\
\frac{3}{2}+b \varepsilon
\end{array} \mid \chi\right), \\
& { }_{2} F_{1}\left(\begin{array}{c}
1, \frac{1}{2} \\
2-\varepsilon
\end{array} \mid u\right)=\frac{1}{1-2 \varepsilon}\left[2(1-\varepsilon)-(1-u){ }_{2} F_{1}\left(\begin{array}{c}
1, \frac{3}{2} \mid \\
2-\varepsilon
\end{array} \mid u\right)\right]=(1+\chi){ }_{2} F_{1}\left(\begin{array}{c}
1, \varepsilon \\
2-\varepsilon
\end{array} \mid \chi\right),
\end{aligned}
$$

where

$$
\chi=\frac{1-\sqrt{1-u}}{1+\sqrt{1-u}}, \quad u=\frac{4 \chi}{(1+\chi)^{2}},
$$

and all orders of the $\varepsilon$-expansion of the last ${ }_{2} F_{1}$ function on the r.h.s. of Eq. (D.6) are known through Eq. (2.14) of Ref. [16]. 
Combining all these relations together with the results for the multiple inverse binomial sums presented in this paper, we have reproduced several known results for multiple binomial sums, including those obtained in Ref. [8]. For completeness, we list a number of such results, including trivial ones, which could be extracted from Ref. [8] (although were not explicitly listed there),

$$
\begin{aligned}
& \sum_{j=1}^{\infty}\left(\begin{array}{c}
2 j \\
j
\end{array}\right) z^{j}=\frac{2 \chi}{1-\chi} \\
& \sum_{j=1}^{\infty}\left(\begin{array}{c}
2 j \\
j
\end{array}\right) \frac{z^{j}}{j}=2 \ln (1+\chi) \\
& \sum_{j=1}^{\infty}\left(\begin{array}{c}
2 j \\
j
\end{array}\right) \frac{z^{j}}{j^{2}}=-2 \operatorname{Li}_{2}(-\chi)-2 \ln ^{2}(1+\chi), \\
& \sum_{j=1}^{\infty}\left(\begin{array}{c}
2 j \\
j
\end{array}\right) \frac{z^{j}}{j^{3}}=4 \mathrm{~S}_{1,2}(-\chi)-2 \operatorname{Li}_{3}(-\chi)+4 \operatorname{Li}_{2}(-\chi) \ln (1+\chi)+\frac{4}{3} \ln ^{3}(1+\chi), \\
& \sum_{j=1}^{\infty}\left(\begin{array}{c}
2 j \\
j
\end{array}\right) z^{j} S_{1}=-\frac{2}{1-\chi}[(1-\chi) \ln (1+\chi)+(1+\chi) \ln (1-\chi)], \\
& \sum_{j=1}^{\infty}\left(\begin{array}{c}
2 j \\
j
\end{array}\right) \frac{z^{j}}{j} S_{1}=\operatorname{Li}_{2}\left(\chi^{2}\right)+2 \ln ^{2}(1+\chi), \\
& \sum_{j=1}^{\infty}\left(\begin{array}{c}
2 j \\
j
\end{array}\right) z^{j} \bar{S}_{1}=\frac{2}{1-\chi}[\chi \ln (1+\chi)-(1+\chi) \ln (1-\chi)] \\
& \sum_{j=1}^{\infty}\left(\begin{array}{c}
2 j \\
j
\end{array}\right) \frac{z^{j}}{j} \bar{S}_{1}=2 \mathrm{Li}_{2}(\chi)+\ln ^{2}(1+\chi), \\
& \sum_{j=1}^{\infty}\left(\begin{array}{c}
2 j \\
j
\end{array}\right) z^{j} S_{2}=-\frac{4 \chi}{1-\chi} \operatorname{Li}_{2}(-\chi)+2 \ln ^{2}(1+\chi), \\
& \sum_{j=1}^{\infty}\left(\begin{array}{c}
2 j \\
j
\end{array}\right) z^{j} S_{1}^{2}=\frac{2}{1-\chi}\left[4 \chi \operatorname{Li}_{2}(\chi)+2 \chi \operatorname{Li}_{2}(-\chi)-(1-\chi) \ln ^{2}(1+\chi)+2(1+\chi) \ln ^{2}(1-\chi)\right], \\
& \sum_{j=1}^{\infty}\left(\begin{array}{c}
2 j \\
j
\end{array}\right) z^{j} S_{1} \bar{S}_{1}=\frac{1}{1-\chi}\left[6 \chi \operatorname{Li}_{2}(\chi)-2(1+\chi) \ln (1+\chi) \ln (1-\chi)\right. \\
& \left.-(1-\chi) \ln ^{2}(1+\chi)+4(1+\chi) \ln ^{2}(1-\chi)\right], \\
& \sum_{j=1}^{\infty}\left(\begin{array}{c}
2 j \\
j
\end{array}\right) z^{j}\left(\bar{S}_{2}-\bar{S}_{1}^{2}\right)=-\frac{2}{1-\chi}\left[2 \chi \operatorname{Li}_{2}(\chi)-2(1+\chi) \ln (1+\chi) \ln (1-\chi)\right. \\
& \left.+\chi \ln ^{2}(1+\chi)+2(1+\chi) \ln ^{2}(1-\chi)\right] .
\end{aligned}
$$

Furthermore, explicit results for the multiple binomial sums with $1 / j^{4}, S_{1} / j^{2}, \bar{S}_{1} / j^{2}$, $S_{2} / j, S_{1}^{2} / j, S_{1} \bar{S}_{1} / j$, and $\left(\bar{S}_{2}-\bar{S}_{1}^{2}\right) / j$ are presented in Eqs. (A.4)-(A.10) of Ref. [8] (where the same notation $\chi$ as here was used, with $x$ corresponding to our $z$ ). We confirm all those 
results. Moreover, using our approach we have obtained a number of new results for the multiple binomial sums,

$$
\begin{aligned}
& \sum_{j=1}^{\infty}\left(\begin{array}{c}
2 j \\
j
\end{array}\right) z^{j} S_{3}=-\frac{4}{3} \ln ^{3}(1+\chi)-4 \ln (1+\chi) \operatorname{Li}_{2}(-\chi)-\frac{4}{1-\chi}\left[2 \mathrm{~S}_{1,2}(-\chi)+\chi \operatorname{Li}_{3}(-\chi)\right], \\
& \sum_{j=1}^{\infty}\left(\begin{array}{c}
2 j \\
j
\end{array}\right) z^{j} S_{1} S_{2}=\frac{4}{3} \ln ^{3}(1+\chi)+4 \ln (1+\chi) \operatorname{Li}_{2}(-\chi)+4 \ln (1+\chi) \operatorname{Li}_{2}(\chi) \\
& +\frac{4}{1-\chi}\left[\chi \operatorname{Li}_{3}(\chi)+\mathrm{S}_{1,2}\left(\chi^{2}\right)-2 \mathrm{~S}_{1,2}(\chi)+(1+\chi) \ln (1-\chi) \operatorname{Li}_{2}(-\chi)\right], \\
& \sum_{j=1}^{\infty}\left(\begin{array}{c}
2 j \\
j
\end{array}\right) z^{j} S_{2} \bar{S}_{1}=\frac{2}{3} \ln ^{3}(1+\chi)+4 \ln (1+\chi) \operatorname{Li}_{2}(\chi) \\
& +\frac{2}{1-\chi}\left[2 \chi \mathrm{Li}_{3}(\chi)-6 \mathrm{~S}_{1,2}(-\chi)+2 \mathrm{~S}_{1,2}\left(\chi^{2}\right)-4 \mathrm{~S}_{1,2}(\chi)-\chi \operatorname{Li}_{3}(-\chi)\right] \\
& +\frac{2(1+\chi)}{1-\chi}[2 \ln (1-\chi)-\ln (1+\chi)] \operatorname{Li}_{2}(-\chi), \\
& \sum_{j=1}^{\infty}\left(\begin{array}{c}
2 j \\
j
\end{array}\right) z^{j} S_{1}\left(\bar{S}_{2}-\bar{S}_{1}^{2}\right)=\frac{2}{1-\chi}\left[16 \mathrm{~S}_{1,2}(\chi)-2 \chi \operatorname{Li}_{3}(\chi)-6 \chi \ln (1+\chi) \operatorname{Li}_{2}(\chi)\right] \\
& +\frac{2(1+\chi)}{1-\chi}\left[4 \ln ^{3}(1-\chi)-4 \ln ^{2}(1-\chi) \ln (1+\chi)\right. \\
& \left.+\ln (1-\chi) \ln ^{2}(1+\chi)+8 \ln (1-\chi) \operatorname{Li}_{2}(\chi)\right]+\frac{2}{3} \ln ^{3}(1+\chi), \\
& \sum_{j=1}^{\infty}\left(\begin{array}{c}
2 j \\
j
\end{array}\right) z^{j} S_{1}^{2} \bar{S}_{1}=-\frac{2}{1-\chi}\left[2 \mathrm{~S}_{1,2}\left(\chi^{2}\right)-6 \mathrm{~S}_{1,2}(-\chi)+16 \mathrm{~S}_{1,2}(\chi)-\chi \operatorname{Li}_{3}(-\chi)\right. \\
& \left.-4 \chi \operatorname{Li}_{3}(\chi)+2(1-3 \chi) \ln (1+\chi) \operatorname{Li}_{2}(\chi)\right] \\
& -\frac{2(1+\chi)}{1-\chi}\left[4 \ln ^{3}(1-\chi)-2 \ln ^{2}(1-\chi) \ln (1+\chi)+2 \ln (1-\chi) \operatorname{Li}_{2}(-\chi)\right. \\
& \left.+10 \ln (1-\chi) \operatorname{Li}_{2}(\chi)-\ln (1+\chi) \operatorname{Li}_{2}(-\chi)\right]-\frac{2}{3} \ln ^{3}(1+\chi), \\
& \sum_{j=1}^{\infty}\left(\begin{array}{c}
2 j \\
j
\end{array}\right) z^{j}\left(3 \bar{S}_{1} \bar{S}_{2}-2 \bar{S}_{3}-\bar{S}_{1}^{3}\right)=\frac{2}{1-\chi}\left[12 \mathrm{~S}_{1,2}(\chi)-6 \chi \ln (1+\chi) \operatorname{Li}_{2}(\chi)-\chi \ln ^{3}(1+\chi)\right] \\
& +\frac{2(1+\chi)}{1-\chi}\left[6 \ln (1-\chi) \operatorname{Li}_{2}(\chi)-6 \ln ^{2}(1-\chi) \ln (1+\chi)\right. \\
& \left.+3 \ln (1-\chi) \ln ^{2}(1+\chi)+4 \ln ^{3}(1-\chi)\right], \\
& \sum_{j=1}^{\infty}\left(\begin{array}{c}
2 j \\
j
\end{array}\right) z^{j} S_{1}^{3}=-\frac{4}{3} \ln ^{3}(1+\chi)-4 \ln (1+\chi) \operatorname{Li}_{2}(-\chi)-12 \ln (1+\chi) \operatorname{Li}_{2}(\chi) \\
& -\frac{4}{1-\chi}\left[3 \mathrm{~S}_{1,2}\left(\chi^{2}\right)-4 \mathrm{~S}_{1,2}(-\chi)+6 \mathrm{~S}_{1,2}(\chi)-2 \chi \operatorname{Li}_{3}(-\chi)-3 \chi \operatorname{Li}_{3}(\chi)\right] \\
& -\frac{4(1+\chi)}{1-\chi}\left[2 \ln ^{3}(1-\chi)+3 \ln (1-\chi) \operatorname{Li}_{2}(-\chi)+6 \ln (1-\chi) \operatorname{Li}_{2}(\chi)\right] \text {, }
\end{aligned}
$$


where $\chi$ is defined in Eq. (D.77) and, as before, $u=4 z$. These results were recently used to construct the $\varepsilon$-expansion of two-loop sunset-type diagrams of special type (for details, see section 3.1 in [62]).

The results for harmonic sums of the type $\sum_{j=1}^{\infty} z^{j} S_{a_{1}} \ldots S_{a_{k}} \bar{S}_{b_{1}} \ldots \bar{S}_{b_{j}}$ could be deduced from the following relation:

$$
\begin{aligned}
{ }_{2} F_{1}\left(\begin{array}{c}
1, \frac{3}{2}+a \varepsilon \\
\frac{3}{2}+b \varepsilon
\end{array} \mid z\right)= & \frac{\Gamma(2+2 b \varepsilon) \Gamma(1+a \varepsilon) \Gamma(1+(a-b) \varepsilon)}{\Gamma(1+b \varepsilon) \Gamma(2+2 a \varepsilon)} \frac{4^{(a-b) \varepsilon}}{(1-z)^{1+(a-b) \varepsilon} z^{1 / 2+b \varepsilon}} \\
& -\frac{1+2 b \varepsilon}{2(1+(a-b) \varepsilon)}{ }_{2} F_{1}\left(\begin{array}{c}
1, \frac{3}{2}+a \varepsilon \\
2+(a-b) \varepsilon
\end{array} \mid 1-z\right),
\end{aligned}
$$

which corresponds to an analytic continuation of the ${ }_{2} F_{1}$ function from the argument $z$ to $(1-z)$. In particular, we get

$$
\begin{aligned}
\sum_{j=1}^{\infty} S_{1} \bar{S}_{1} z^{j}= & \frac{z}{1-z}\left\{\frac{1}{4} \ln ^{2}(1-z)+\frac{1}{2} \ln ^{2}(1-\sqrt{z})+\frac{1}{2} \ln ^{2}(1+\sqrt{z})+\frac{1}{2} \operatorname{Li}_{2}(z)\right. \\
& +\frac{1}{2 \sqrt{z}}\left[\operatorname{Li}_{2}\left(\frac{1+\sqrt{z}}{2}\right)-\operatorname{Li}_{2}\left(\frac{1-\sqrt{z}}{2}\right)\right] \\
& \left.\left.+\frac{1}{4 \sqrt{z}}\left[\ln ^{2}(1-\sqrt{z})-\ln ^{2}(1+\sqrt{z})\right]-\frac{\ln 2}{2 \sqrt{z}} \ln \left(\frac{1+\sqrt{z}}{1-\sqrt{z}}\right)\right]\right\} .
\end{aligned}
$$

Introducing a new variable $\xi$ such that

$$
\xi=\frac{1-\sqrt{z}}{1+\sqrt{z}}, \quad z=\frac{(1-\xi)^{2}}{(1+\xi)^{2}}
$$

we can present the above result in a more compact form,

$$
\sum_{j=1}^{\infty} S_{1} \bar{S}_{1} z^{j}=\frac{z}{2(1-z)}\left[\operatorname{Li}_{2}(z)+\ln ^{2}(1-z)\right]+\frac{1-\xi^{2}}{4 \xi}\left[\operatorname{Li}_{2}(-\xi)+\ln \xi \ln 2+\frac{1}{2} \zeta_{2}\right]+\frac{1-\xi}{8 \xi} \ln ^{2} \xi
$$

where we have taken into account that

$$
\operatorname{Li}_{2}\left(\frac{1+\sqrt{z}}{2}\right)-\operatorname{Li}_{2}\left(\frac{1-\sqrt{z}}{2}\right)=2 \operatorname{Li}_{2}(-\xi)+\ln \xi \ln (1+\xi)+\zeta_{2} .
$$

Integrating the representation (D.13), it is easy to get the following result:

$$
\begin{aligned}
\sum_{j=1}^{\infty} S_{1} \bar{S}_{1} \frac{z^{j}}{j}= & -\mathrm{S}_{1,2}(z)-\frac{1}{2} \ln (1-z) \operatorname{Li}_{2}(z)-\frac{1}{6} \ln ^{3}(1-z)-\frac{3}{2} \zeta_{3}-\frac{1}{4} \ln ^{2} \xi \ln (1-z) \\
& -2 \mathrm{Li}_{3}(-\xi)+\mathrm{Li}_{2}(-\xi) \ln \xi-\frac{1}{2} \zeta_{2} \ln \xi+\frac{1}{12} \ln ^{3} \xi
\end{aligned}
$$

In Appendix E of Ref. [14] the results (up to weight 4) for the harmonic sums of the type $\sum_{j=1}^{\infty} S_{a_{1}} \ldots S_{a_{k}} z^{j} / j^{a}$ have been presented. It is easy to extend those results to the case $\sum_{j=1}^{\infty} \bar{S}_{a_{1}} \ldots \bar{S}_{a_{k}} z^{j} / j^{a}$, using the following property [32]:

$$
\text { if } \sum_{j=1}^{\infty} f(j) \frac{z^{j}}{j^{a}}=F(z) \quad \text { then } \sum_{j=1}^{\infty} f(2 j) \frac{z^{j}}{j^{a}}=2^{a-1}[F(\sqrt{z})+F(-\sqrt{z})] \text {. }
$$


As a consequence, we get

$$
\begin{aligned}
\sum_{j=1}^{\infty} \bar{S}_{a} z^{j} & =\frac{\sqrt{z}}{2(1-\sqrt{z})} \operatorname{Li}_{a}(\sqrt{z})-\frac{\sqrt{z}}{2(1+\sqrt{z})} \operatorname{Li}_{a}(-\sqrt{z}), \\
\sum_{j=1}^{\infty} \bar{S}_{1} \frac{z^{j}}{j^{a}} & =2^{a-1}\left[\mathrm{~S}_{a-1,2}(\sqrt{z})+\mathrm{S}_{a-1,2}(-\sqrt{z})\right] .
\end{aligned}
$$

As a further illustration, we also present results for other sums up to weight $\mathbf{3}$,

$$
\begin{aligned}
\sum_{j=1}^{\infty} \bar{S}_{1}^{2} z^{j}= & \frac{\sqrt{z}}{2(1-\sqrt{z})}\left[\operatorname{Li}_{2}(\sqrt{z})+\ln ^{2}(1-\sqrt{z})\right]-\frac{\sqrt{z}}{2(1+\sqrt{z})}\left[\operatorname{Li}_{2}(-\sqrt{z})+\ln ^{2}(1+\sqrt{z})\right] \\
\sum_{j=1}^{\infty} \bar{S}_{2} \frac{z^{j}}{j}= & -\ln (1-\sqrt{z}) \operatorname{Li}_{2}(\sqrt{z})-\ln (1+\sqrt{z}) \operatorname{Li}_{2}(-\sqrt{z})-2 \mathrm{~S}_{1,2}(\sqrt{z})-2 \mathrm{~S}_{1,2}(-\sqrt{z}), \\
\sum_{j=1}^{\infty} \bar{S}_{1}^{3} z^{j}= & \frac{\sqrt{z}}{2(1-\sqrt{z})}\left[\operatorname{Li}_{3}(\sqrt{z})-3 \ln (1-\sqrt{z}) \operatorname{Li}_{2}(\sqrt{z})+\ln ^{3}(1-\sqrt{z})-3 \mathrm{~S}_{1,2}(\sqrt{z})\right] \\
& -\frac{\sqrt{z}}{2(1+\sqrt{z})}\left[\operatorname{Li}_{3}(-\sqrt{z})-3 \ln (1+\sqrt{z}) \operatorname{Li}_{2}(-\sqrt{z})+\ln ^{3}(1+\sqrt{z})-3 \mathrm{~S}_{1,2}(-\sqrt{z})\right], \\
\sum_{j=1}^{\infty} \bar{S}_{1}^{2} \frac{z^{j}}{j}= & -\ln (1-\sqrt{z}) \operatorname{Li}_{2}(\sqrt{z})-\ln (1+\sqrt{z}) \operatorname{Li}_{2}(-\sqrt{z})-2 \mathrm{~S}_{1,2}(\sqrt{z})-2 \mathrm{~S}_{1,2}(-\sqrt{z}) \\
& +\frac{1}{3} \ln (1-\sqrt{z})+\frac{1}{3} \ln (1+\sqrt{z}), \\
\sum_{j=1}^{\infty} \bar{S}_{1} \bar{S}_{2} z^{j}= & \frac{\sqrt{z}}{2(1-\sqrt{z})}\left[\operatorname{Li}_{3}(\sqrt{z})-\ln (1-\sqrt{z}) \operatorname{Li}_{2}(\sqrt{z})-\mathrm{S}_{1,2}(\sqrt{z})\right] \\
& -\frac{\sqrt{z}}{2(1+\sqrt{z})}\left[\operatorname{Li}_{3}(-\sqrt{z})-\ln (1+\sqrt{z}) \operatorname{Li}_{2}(-\sqrt{z})-\mathrm{S}_{1,2}(-\sqrt{z})\right] .
\end{aligned}
$$

To investigate relations between multiple harmonic sums the technique proposed in Ref. [63] can be useful.

As a illustration, we present the hihger order $\varepsilon$-expansion of the some of the hypergeometric functions:

$$
\begin{aligned}
& { }_{2} F_{1}\left(\begin{array}{c}
1+a_{1} \varepsilon, 1+a_{2} \varepsilon \\
2+c \varepsilon
\end{array} \mid z\right)=\frac{1+c \varepsilon}{z}\left(-\ln (1-z)-\varepsilon\left\{\frac{c-a_{1}-a_{2}}{2} \ln ^{2}(1-z)+c \operatorname{Li}_{2}(z)\right\}\right. \\
& +\varepsilon^{2}\left\{\left[\left(a_{1}+a_{2}\right) c-c^{2}-2 a_{1} a_{2}\right] \mathrm{S}_{1,2}(z)+\left[\left(a_{1}+a_{2}\right) c-c^{2}-a_{1} a_{2}\right] \ln (1-z) \operatorname{Li}_{2}(z)\right. \\
& \left.\quad+c^{2} \operatorname{Li}_{3}(z)-\frac{\left(c-a_{1}-a_{2}\right)^{2}}{6} \ln ^{3}(1-z)\right\} \\
& -\varepsilon^{3}\left\{c\left[\left(a_{1}+a_{2}\right) c-c^{2}-2 a_{1} a_{2}\right] \mathrm{S}_{2,2}(z)+c\left[\left(a_{1}+a_{2}\right) c-c^{2}-a_{1} a_{2}\right] \ln (1-z) \operatorname{Li}_{3}(z)\right. \\
& \quad+\left(c-a_{1}\right)\left(c-a_{2}\right)\left(c-a_{1}-a_{2}\right)\left[\ln (1-z) \mathrm{S}_{1,2}(z)+\frac{1}{2} \ln ^{2}(1-z) \operatorname{Li}_{2}(z)\right]
\end{aligned}
$$




$$
\left.\left.+\frac{\left(c-a_{1}-a_{2}\right)^{3}}{24} \ln ^{4}(1-z)+c\left(c-a_{1}-a_{2}\right)^{2} \mathrm{~S}_{1,3}(z)+c^{3} \operatorname{Li}_{4}(z)\right\}+\mathcal{O}\left(\varepsilon^{4}\right)\right)
$$

\section{E The $\mathcal{O}\left(\alpha \alpha_{\mathrm{s}}\right)$ corrections to the polarization function of neutral gauge bosons in arbitrary dimension}
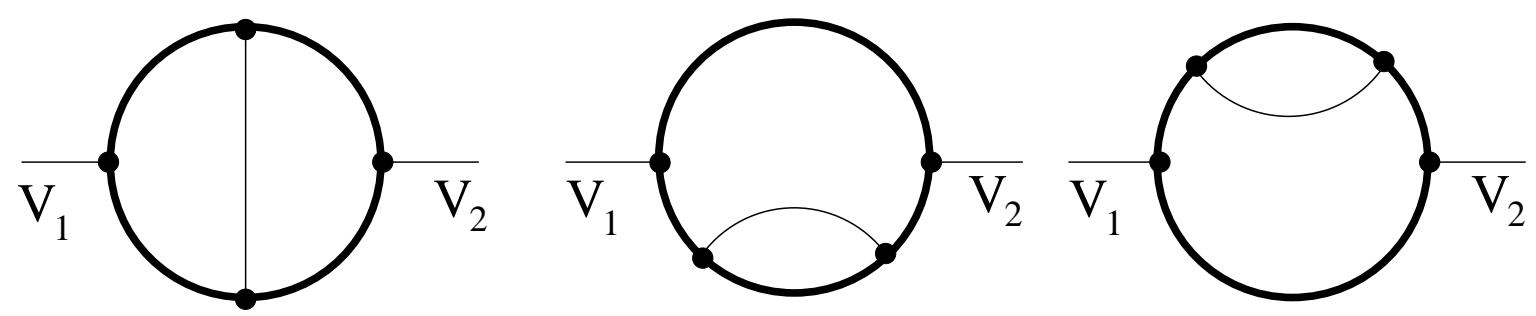

Figure 2: Two-loop contributions to the off-shell polarization function of a neutral gauge boson. Bold and thin lines correspond to the massive quark propagator and the massless boson (gluon or photon) propagator, respectively.

Here we present an example of a physically relevant calculation ${ }^{11}$ that can be expressed in terms of the master integrals $J_{011}$ studied in Section 4.3. Let us consider the two-loop propagator-type diagrams shown in Fig. 2. All of these $\mathcal{O}\left(\alpha \alpha_{\mathrm{s}}\right)$ contributions to the polarization function of the gauge bosons involve a quark loop with a gluon exchange. It was analytically calculated in [56], up to the finite term of the $\varepsilon$-expansion. Here we present the bare two-loop results in $n$-dimensional space-time (see also in Ref. [8]). In contrast to the calculations performed in [56], here we use Tarasov's recurrence relations [38] for the reduction of the original integrals to the set of master integrals. In this Appendix we use the Euclidean notation [57], $P^{2} \leftrightarrow-p^{2}$, so that the on-shell limit would read $P^{2} \rightarrow-m^{2}$.

Let us decompose the polarization tensor into the transverse $\Pi_{\mathrm{T}}\left(P^{2}\right)$ and longitudinal $\Pi_{\mathrm{L}}\left(P^{2}\right)$ parts,

$$
\Pi_{\mu \nu}\left(P^{2}\right)=\left(\delta_{\mu \nu}-\frac{P_{\mu} P_{\nu}}{P^{2}}\right) \Pi_{\mathrm{T}}\left(P^{2}\right)+\frac{P_{\mu} P_{\nu}}{P^{2}} \Pi_{\mathrm{L}}\left(P^{2}\right) .
$$

Then, the two-loop corrections can be written as

$$
\begin{aligned}
& \Pi_{\mathrm{T}}^{(2)}(\left.P^{2}\right)=\frac{g^{2} g_{\mathrm{s}}^{2}}{(4 \pi)^{n / 2}} N_{c} C_{F}\left\{-J_{011}(1,1,1) \frac{4}{(n-1) t}\left[-4 A n m^{2}+12 A m^{2}+A t n^{2}\right.\right. \\
&\left.-3 A t n+2 A t+S t n^{2}-3 S t n+2 S t\right] \\
&- J_{011}(1,1,2) \frac{4}{(n-4)(n-3)(n-1) t}\left[112 A m^{4} n+A t^{2} n^{3}-7 A t^{2} n^{2}+18 A t^{2} n\right. \\
&+S t^{2} n^{3}-7 S t^{2} n^{2}+18 S t^{2} n-16 A m^{4} n^{2}-192 A m^{4}+4 A m^{2} t n^{3}-32 A m^{2} t n^{2}-16 A t^{2}
\end{aligned}
$$

\footnotetext{
${ }^{11}$ Another non-trivial example where these master integrals appear is given in Ref. [55].
} 


$$
\begin{aligned}
& \left.+100 A m^{2} t n+4 S m^{2} t n^{3}-36 S m^{2} t n^{2}+96 S m^{2} t n-112 A m^{2} t-16 S t^{2}-80 S m^{2} t\right] \\
& +\left[A_{0}(m)\right]^{2} \frac{2(n-2)}{\left(4 m^{2}+t\right) m^{2} t(n-4)(n-1)(n-3)}\left[-4 S t n^{4} m^{2}+A t^{2} n^{3}+4 A m^{2} t n^{3}\right. \\
& +36 S m^{2} t n^{3}+S t^{2} n^{3}-32 A m^{2} t n^{2}-7 S t^{2} n^{2}-16 A m^{4} n^{2}-120 S m^{2} t n^{2}-7 A t^{2} n^{2} \\
& +112 A m^{4} n+184 S m^{2} t n+100 A m^{2} t n+18 A t^{2} n+18 S t^{2} n-192 A m^{4}-16 A t^{2} \\
& \left.-112 S m^{2} t-112 A m^{2} t-16 S t^{2}\right] \\
& -A_{0}(m) B_{0}(m, m, t) \frac{2(n-2)}{m^{2}(n-3)\left(4 m^{2}+t\right)(n-4)(n-1)}\left[432 A m^{4} n-2 A t n^{4} m^{2}\right. \\
& +64 m^{4} S+40 m^{4} S n+8 m^{4} S n^{3}-48 m^{4} S n^{2}+80 A m^{4} n^{3}-8 A n^{4} m^{4}-2 S t n^{4} m^{2} \\
& +A t^{2} n^{3}-7 A t^{2} n^{2}+18 A t^{2} n+S t^{2} n^{3}-7 S t^{2} n^{2}+18 S t^{2} n-280 A m^{4} n^{2}-256 A m^{4} \\
& +24 A m^{2} t n^{3}-98 A m^{2} t n^{2}+180 A m^{2} t n+24 S m^{2} t n^{3}-102 S m^{2} t n^{2}+176 S m^{2} t n \\
& \left.-16 A t^{2}-128 A m^{2} t-16 S t^{2}-96 S m^{2} t\right] \\
& -\left[B_{0}(m, m, t)\right]^{2} \frac{2}{\left(4 m^{2}+t\right)(n-4)(n-1)}\left[448 A m^{4} n-32 m^{4} S+16 A m^{4} n^{3}\right. \\
& +A t^{2} n^{3}-9 A t^{2} n^{2}+30 A t^{2} n+S t^{2} n^{3}-9 S t^{2} n^{2}+30 S t^{2} n-144 A m^{4} n^{2}-448 A m^{4} \\
& +8 A m^{2} t n^{3}-72 A m^{2} t n^{2}+232 A m^{2} t n+4 S m^{2} t n^{3}-44 S m^{2} t n^{2}+152 S m^{2} t n \\
& \left.\left.-32 A t^{2}-240 A m^{2} t-32 S t^{2}-160 S m^{2} t\right]\right\} \\
& \Pi_{\mathrm{L}}^{(2)}\left(P^{2}\right)=A \frac{g^{2} g_{\mathrm{s}}^{2}}{(4 \pi)^{n / 2}} N_{c} C_{F}\left\{-J_{011}(1,1,1) \frac{16 m^{2}(n-3)}{t}\right. \\
& -J_{011}(1,1,2) \frac{16 m^{2}}{t(n-4)(n-3)}\left[4 m^{2} n^{2}-28 m^{2} n+48 m^{2}+t n^{2}-5 t n+8 t\right] \\
& +\left[A_{0}(m)\right]^{2} \frac{8(n-2)}{t\left(4 m^{2}+t\right)(n-4)}\left[t n^{2}-3 t n+4 m^{2} n-16 m^{2}\right] \\
& -\left[B_{0}(m, m, t)\right]^{2} \frac{8 m^{2}}{\left(4 m^{2}+t\right)(n-4)}\left[-4 t n+4 t+4 m^{2} n^{2}-24 m^{2} n+t n^{2}+40 m^{2}\right] \\
& \left.-A_{0}(m) B_{0}(m, m, t) \frac{8(n-2)\left(n^{2}-5 n+8\right)}{\left(4 m^{2}+t\right)(n-4)(n-3)}\left[-2 m^{2} n+10 m^{2}+t\right]\right\} \text {, }
\end{aligned}
$$

where the vertices $V_{j}(j=1,2)$ are defined as $^{12}$

$$
V_{j}=\mathrm{i} g \gamma_{\mu}\left(v_{j}+a_{j} \gamma_{5}\right)
$$

and we have introduced the following notations:

$$
A=a_{1} a_{2}, \quad S=v_{1} v_{2}, \quad t=P^{2},
$$

$N_{c}$ is a color factor (equal to 3 for quark and 1 for lepton), $C_{F}$ is the Casimir operator of the fundamental representation of the Lie algebra (equal to $\frac{4}{3}$ for $S U(3)$ and 1 for QED), and $m$

\footnotetext{
${ }^{12}$ Explicit values of coefficients $v_{j}$ and $a_{j}$ for the Standard Model can be extracted from [57].
} 
is the mass of the loop fermion. Finally, the occurring integrals are defined as

$$
\begin{aligned}
J_{011}\left(\sigma, \nu_{1}, \nu_{2}\right) & =\frac{\pi^{-n}}{\Gamma^{2}\left(3-\frac{n}{2}\right)} \iint \frac{\mathrm{d}^{n} K_{1} \mathrm{~d}^{n} K_{2}}{\left[\left(K_{2}-P\right)^{2}\right]^{\sigma}\left[K_{1}^{2}+m^{2}\right]^{\nu_{1}}\left[\left(K_{1}-K_{2}\right)^{2}+m^{2}\right]^{\nu_{2}}}, \\
B_{0}\left(m_{a}, m_{b}, t\right) & =\frac{\pi^{-n / 2}}{\Gamma\left(3-\frac{n}{2}\right)} \int \frac{\mathrm{d}^{n} K}{\left[(K-P)^{2}+m_{a}^{2}\right]\left[K^{2}+m_{b}^{2}\right]}, \\
A_{0}(m) & =\frac{\pi^{-n / 2}}{\Gamma\left(3-\frac{n}{2}\right)} \int \frac{\mathrm{d}^{n} K}{K^{2}+m^{2}} \equiv \frac{4\left(m^{2}\right)^{n-2}}{(n-2)(n-4)} .
\end{aligned}
$$

We note that the integral $J_{011}$ is defined in the same way as in Section 4.3, one should only remember to substitute $p^{2} \rightarrow-t$.

In particular, for the zero momentum transfer, $P^{2}=t=0$, we get

$$
\begin{aligned}
& \Pi_{\mathrm{L}}^{(2)}(0)=-A \frac{g^{2} g_{\mathrm{s}}^{2}}{(4 \pi)^{n / 2}} N_{c} C_{F}\left[A_{0}(m)\right]^{2} \frac{4(n-2)\left(n^{2}-5 n+7\right)}{m^{2}} \\
& \Pi_{\mathrm{T}}^{(2)}(0)=-A \frac{g^{2} g_{s}^{2}}{(4 \pi)^{n / 2}} N_{c} C_{F}\left[A_{0}(m)\right]^{2} \frac{4(n-2)\left(n^{3}-6 n^{2}+13 n-11\right)}{(n-1) m^{2}}
\end{aligned}
$$

To obtain the finite terms of the $\varepsilon$-expansion of the results given in Eqs. (E.1) and (E.2), the integral $J_{011}(1,1,2)$ should be expanded up to the $\varepsilon$-part. Furthermore, using the approach of Ref. [38], we obtain the following relations between the integrals investigated in Section 4.3 and the master integrals $J_{011}(1,1,1)$ and $J_{011}(1,1,2)$ :

$$
\begin{aligned}
& J_{011}(1,1,1) \frac{(3 n-8)(3 n-10)(n-3)^{2}}{n-4}=\left[A_{0}(m)\right]^{2}(n-3)(n-2)^{2}\left[\frac{t}{4 m^{4}}-\frac{(7 n-24)}{2 m^{2}(n-4)}\right] \\
& \quad+J_{011}(1,2,2)\left[\frac{8 m^{2}\left(t+m^{2}\right)}{n-4}-2 t^{2}(n-3)+2 t m^{2}(7 n-17)+8 m^{4}(2 n-5)\right] \\
& \quad+\left[J_{011}(1,2,2)+2 J_{011}(2,1,2)\right](n-3)\left(t+4 m^{2}\right)\left(t-2 m^{2}\right) \\
& J_{011}(1,1,2) \frac{(3 n-8)(3 n-10)(n-3)^{2}}{n-4}=\left[A_{0}(m)\right]^{2} \frac{(n-3)(2 n-7)(3 n-8)(n-2)^{2}}{4 m^{4}(n-4)} \\
& \quad+J_{011}(1,2,2)\left[-\frac{4\left(t+2 m^{2}\right)}{n-4}-n^{2}\left(\frac{15}{2} t+12 m^{2}\right)+n\left(\frac{79}{2} t+62 m^{2}\right)-55 t-86 m^{2}\right] \\
& \quad+\frac{1}{2}\left[J_{011}(1,2,2)+2 J_{011}(2,1,2)\right](n-3)(3 n-8)\left(t+4 m^{2}\right) .
\end{aligned}
$$

From these relations we see that, in order to obtain the $\varepsilon^{j}$ terms of $J_{011}(1,1,1)$ and $J_{011}(1,1,2)$, the integral $J_{011}(1,2,2)$ should be expanded up to $\varepsilon^{j}$, whereas the combination $\left[J_{011}(1,2,2)+\right.$ $\left.2 J_{011}(2,1,2)\right]$ up to $\varepsilon^{j-1}$ only. The expansion of the integrals near the threshold can be performed by using the numerical algorithm described in Ref. [58].

The higher-order terms of the $\varepsilon$-expansion of the one-loop integral $B_{0}\left(m_{1}, m_{2}, t\right)$ can be extracted from Refs. [16,30]. Here we present coefficients up to the order $\varepsilon^{2}$ for the particular 
case $m_{1}=m_{2}=m$ :

$$
\begin{aligned}
B_{0}(m, m, t)= & \frac{\left(m^{2}\right)^{-\varepsilon}}{(1-2 \varepsilon)}\left(\frac{1}{\varepsilon}+\frac{1+y}{1-y}\left\{\ln y+\varepsilon\left[\frac{1}{2} \ln ^{2} y-2 \ln y \ln (1+y)-2 \operatorname{Li}_{2}(-y)-\zeta_{2}\right]\right.\right. \\
& +\varepsilon^{2}\left[4 \mathrm{~S}_{1,2}(-y)-2 \operatorname{Li}_{3}(-y)+4 \ln (1+y) \operatorname{Li}_{2}(-y)-\ln ^{2} y \ln (1+y)\right. \\
& \left.\left.\left.+2 \ln y \ln ^{2}(1+y)+\frac{1}{6} \ln ^{3} y-\zeta_{2} \ln y+2 \zeta_{2} \ln (1+y)-2 \zeta_{3}\right]+\mathcal{O}\left(\varepsilon^{3}\right)\right\}\right),
\end{aligned}
$$

where the variable $y$ is defined in Section 3.

The bare two-loop amplitudes (E.1) and (E.2) contain subdivergencies which should be canceled by proper counterterms,

$$
\Pi_{\mathrm{CT}}\left(P^{2}\right)=\delta m^{2} \frac{\partial}{\partial m^{2}} \Pi^{(1)}\left(P^{2}\right)
$$

where $\Pi^{(1)}$ is the one-loop amplitude, while $\delta m^{2}$ is the one-loop mass counterterm defined in a particular renormalization scheme. The derivatives of the bare one-loop amplitudes in $n$ dimensions read

$$
\begin{aligned}
& \frac{\partial}{\partial m^{2}} \Pi_{\mathrm{T}}^{(1)}\left(P^{2}\right)=\frac{g^{2}}{(4 \pi)^{n / 2}} N_{c} 4\left\{\left[\frac{S t(3-n)}{\left(4 m^{2}+t\right)}+S+(2-n) A\right] B_{0}(m, m, t)+\frac{2 S(n-2)}{\left(4 m^{2}+t\right)} A_{0}(m)\right\} \\
& \frac{\partial}{\partial m^{2}} \Pi_{\mathrm{L}}^{(1)}\left(P^{2}\right)=A \frac{g^{2}}{(4 \pi)^{n / 2}} N_{c} 4\left\{\left[\frac{t(n-3)}{\left(4 m^{2}+t\right)}+1-n\right] B_{0}(m, m, t)+\frac{2(2-n)}{\left(4 m^{2}+t\right)} A_{0}(m)\right\} .
\end{aligned}
$$

As an example of application of these formulae, let us consider the transversal part of the $\gamma-Z$ propagator $(A=0)$ in the $\overline{M S}$-scheme. Up to the finite in $\varepsilon$ part, the result for the subtracted quantity $\Pi^{\mathrm{sub}}\left(P^{2}\right)=\Pi^{(2)}\left(P^{2}\right)+\Pi_{\mathrm{CT}}$ is

$$
\begin{aligned}
\Pi_{\mathrm{T}, \gamma Z}^{\mathrm{sub}}\left(P^{2}\right)= & S \frac{g^{2} g_{\mathrm{s}}^{2}}{(4 \pi)^{2}} N_{c} C_{F}\left\{-\frac{2 t}{\varepsilon}+\left(-\frac{55}{3} t+\frac{296}{3} m^{2}\right)+16 t \zeta_{3}\left[1-4 \frac{y^{2}}{(1-y)^{4}}\right]\right. \\
& -\frac{16}{3} m^{2} \frac{\left(1-4 y+y^{2}\right)}{y(1-y)^{2}}[\ln (1-y)+2 \ln (1+y)] \ln y\left[\left(1+y^{2}\right) \ln y-2\left(1-y^{2}\right)\right] \\
& +\frac{8}{3} m^{2} \frac{\left(2+7 y-22 y^{2}+6 y^{3}\right)}{(1-y)^{2}} \ln ^{2} y-4 m^{2} \ln y \frac{\left(1-6 y-46 y^{2}-6 y^{3}+y^{4}\right)}{y\left(1-y^{2}\right)} \\
& +4 t \ln \frac{m^{2}}{\mu^{2}}\left[1-\frac{12 y}{(1-y)^{2}}\right]-96 m^{2} \frac{y}{1-y^{2}} \ln y \ln \frac{m^{2}}{\mu^{2}} \\
& +\frac{32}{3} m^{2} \frac{\left(1-4 y+y^{2}\right)}{y(1-y)^{2}}\left[\operatorname{Li}_{2}(y)+2 \mathrm{Li}_{2}(-y)\right]\left[1-y^{2}-2\left(1+y^{2}\right) \ln y\right] \\
& \left.+32 m^{2} \frac{\left(1-4 y+y^{2}\right)}{y(1-y)^{2}}\left(1+y^{2}\right)\left[\operatorname{Li}_{3}(y)+2 \operatorname{Li}_{3}(-y)\right]+\mathcal{O}(\varepsilon)\right\}
\end{aligned}
$$

where we have taken into account the one-loop massive counterterm

$$
\delta m^{2}=-2 \frac{g_{\mathrm{s}}^{2}}{(4 \pi)^{2}} C_{F} \frac{3}{\varepsilon} m^{2} .
$$


The small-momentum expansion of Eq. (E.7) is

$$
\left.\Pi_{T, \gamma Z}^{s u b}\left(p^{2}\right)\right|_{P^{2} \rightarrow 0}=S \frac{g^{2} g_{\mathrm{s}}^{2}}{(4 \pi)^{2}} m^{2} N_{c} C_{F}\left\{\frac{2}{\varepsilon} u+\frac{13}{3} u+\frac{776}{405} u^{2}+\ln \frac{m^{2}}{\mu^{2}}\left(4 u+\frac{8}{5} u^{2}\right)+\mathcal{O}\left(u^{3}\right)\right\}
$$

where $u=-P^{2} / m^{2}$. Let us remind that in a theory with the spontaneous symmetry breaking, like the Standard Model, the inclusion of tadpoles [59] is also required for the renormalization group invariance of the massive parameters $[8,58,60]$. The proper bare two-loop tadpole contribution is given in Section 4.3 of Ref. [8].

\section{References}

[1] G. 'tHooft and M. Veltman, Nucl. Phys. B44 (1972) 189;

C.G. Bollini and J.J. Giambiagi, Nuovo Cim. 12B (1972) 20;

J.F. Ashmore, Lett. Nuovo Cim. 4 (1972) 289;

G.M. Cicuta and E. Montaldi, Lett. Nuovo Cim. 4 (1972) 329.

[2] L. Lewin, Polylogarithms and associated functions (North-Holland, Amsterdam, 1981).

[3] K.S. Kölbig, J.A. Mignaco and E. Remiddi, B.I.T. 10 (1970) 38;

R. Barbieri, J.A. Mignaco and E. Remiddi, Nuovo Cim. A11 (1972) 824;

A. Devoto and D.W. Duke, Riv. Nuovo Cim. 7, No.6 (1984) 1;

K.S. Kölbig, SIAM J. Math. Anal. 17 (1986) 1232.

[4] E. Remiddi and J.A.M. Vermaseren, Int. J. Mod. Phys. A15 (2000) 725.

[5] A.B. Goncharov, Math. Res. Lett. 5 (1998) 497;

J.M. Borwein, D.M. Bradley, D.J. Broadhurst and P. Lisoněk, Trans. Amer. Math. Soc. 353 (2001) 907;

T. Gehrmann and E. Remiddi, Nucl. Phys. B601 (2001) 248.

[6] S. Moch, P. Uwer and S. Weinzierl, J. Math. Phys. 43 (2002) 3363.

[7] S. Weinzierl, hep-ph/0402131.

[8] F. Jegerlehner, M.Yu. Kalmykov and O. Veretin, Nucl. Phys. B658 (2003) 49.

[9] A.V. Kotikov, Phys. Lett. B254 (1991) 158; B259 (1991) 314.

[10] E.E. Boos and A.I. Davydychev, Teor. Mat. Fiz. 89 (1991) 56.

[11] A.P. Isaev, Nucl. Phys. B662 (2003) 461.

[12] J. Fleischer, A.V. Kotikov and O.L. Veretin, Phys. Lett. B417 (1998) 163.

[13] A.I. Davydychev and R. Delbourgo, J. Math. Phys. 39 (1998) 4299. 
[14] J. Fleischer, A.V. Kotikov and O.L. Veretin, Nucl. Phys. B547 (1999) 343.

[15] A.I. Davydychev and M.Yu. Kalmykov, Nucl. Phys. B (Proc. Suppl.) 89 (2000) 283.

[16] A.I. Davydychev and M.Yu. Kalmykov, Nucl. Phys. B605 (2001) 266.

[17] J. Fleischer, F. Jegerlehner and O.V. Tarasov, Nucl. Phys. B672 (2003) 303.

[18] J. Fleischer, M.Yu. Kalmykov and A.V. Kotikov, Phys. Lett. B462 (1999) 169; B467 (1999) 310(E).

[19] D.I. Kazakov and A.V. Kotikov, Theor. Math. Phys. 73 (1988) 1264; Nucl. Phys. B307 (1988) 721;

D. Borwein, J.M. Borwein and R. Girgensohn, Proc. Edinb. Math. Soc. 38 (1995) 277;

J.M. Borwein and R. Girgensohn, Electron. J. Combin. 3 (1996) R23;

P. Flajolet and B. Salvy, Experimental Math. 7 (1998) 15;

O.M. Ogreid and P. Osland, J. Comput. Appl. Math. 98 (1998) 245; 136 (2001) 389;

V.A. Smirnov, Phys. Lett. B547 (2002) 239.

[20] M.Yu. Kalmykov and O. Veretin, Phys. Lett. B483 (2000) 315;

J.M. Borwein and R. Girgensohn, "Evaluation of Binomial Series", CECM preprint CECM-02-188; available at http://www.cecm.sfu.ca/preprints/2002pp.html.

[21] J. Fleischer and M.Yu. Kalmykov, Phys. Lett. B470 (1999) 168.

[22] D.J. Broadhurst, Open University preprint OUT-4102-62 (hep-th/9604128).

[23] D.J. Broadhurst, Eur. Phys. J. C8 (1999) 311.

[24] A.I. Davydychev and M.Yu. Kalmykov, Proc. Workshop "CPP2001", Tokyo, Japan, November 2001, KEK Proceedings 2002-11, p. 169 (hep-th/0203212).

[25] N. Batir, Appl. Math. Comput. 147 (2004) 645.

[26] Z. Nan-Yue and K.S. Williams, Pacific J. Math. 168 (1995) 271.

[27] J.M. Borwein, D.J. Broadhurst and J. Kamnitzer, Experimental Math. 10 (2001) 25.

[28] A.I. Davydychev and J.B. Tausk, Phys. Rev. D53 (1996) 7381;

[29] A.I. Davydychev, Proc. Workshop "AIHENP-99", Heraklion, Greece, April 1999 (Parisianou S.A., Athens, 2000), p. 219 (hep-th/9908032);

A.V. Kotikov and L.N. Lipatov, Nucl. Phys. B582 (2000) 19.

[30] A.I. Davydychev, Phys. Rev. D61 (2000) 087701.

[31] A.P. Prudnikov, Yu.A. Brychkov and O.I. Marichev, Integrals and Series, v.3: More Special Functions, Gordon and Breach, New York, 1990. 
[32] H.S. Wilf, Generatingfunctionology, Academic Press, London, 1994, http://www. math.upenn.edu/ wilf/DownldGF.html

[33] O.V. Tarasov, Phys. Rev. D54 (1996) 6479; Nucl. Phys. B (Proc. Suppl.) 89 (2000) 237

S. Moch and J.A.M. Vermaseren, Nucl. Phys. B573 (2000) 853;

S. Moch, J.A.M. Vermaseren and A. Vogt, Nucl. Phys. B646 (2002) 181;

S. Laporta, Int. J. Mod. Phys. A15 (2000) 5087; Phys. Lett. B504 (2001) 188; B549 (2002) 115.

[34] D.J. Broadhurst, J. Fleischer and O.V. Tarasov, Z. Phys. C60 (1993) 287.

[35] D.J. Broadhurst, Z. Phys. C47 (1990) 115.

[36] A.I. Davydychev, "Loop calculations in QCD with massive quarks", talk at Int. Conf. "Relativistic Nuclear Dynamics" (Vladivostok, Russia, September 1991), http://wwwthep.physik.uni-mainz.de//davyd/preprints/vladiv.ps.gz

[37] A. Bashir, R. Delbourgo and M. L. Roberts, J. Math. Phys. 42 (2001) 5553.

[38] O.V. Tarasov, Nucl. Phys. B502 (1997) 455.

[39] A.I. Davydychev and V.A. Smirnov, Nucl. Phys. B554 (1999) 391.

[40] O.V. Tarasov, Phys. Rev. D54 (1996) 6479.

[41] J. Fleischer, V.A. Smirnov, A. Frink, J.G. Körner, D. Kreimer, K. Schilcher and J.B. Tausk, Eur. Phys. J. C2 (1998) 747.

[42] A. Djouadi, M. Spira and P.M. Zerwas, Phys. Lett. B264 (1991) 440;

S. Dawson and R. Kauffman, Phys. Rev. D49 (1994) 2298;

M. Spira, A. Djouadi, D. Graudenz and P.M. Zerwas, Nucl. Phys. B453 (1995) 17.

[43] G. Passarino and S. Uccirati, Nucl. Phys. B629 (2002) 97;

A. Ferroglia, G. Passarino, S. Uccirati and M. Passera, Nucl. Instrum. Meth. A502 (2003) 391;

A. Ferroglia, M. Passera, G. Passarino and S. Uccirati, Nucl. Phys. B650 (2003) 162;

A. Ferroglia, G. Passarino, M. Passera and S. Uccirati, Nucl. Phys. B680 (2004) 199.

[44] R. Bonciani, P. Mastrolia and E. Remiddi, hep-ph/0311145

[45] R. Bonciani, P. Mastrolia and E. Remiddi, Nucl. Phys. B661 (2003) 289.

[46] V.A. Smirnov, Phys. Lett. B524 (2002) 129.

[47] A.I. Davydychev, P. Osland and L. Saks, Phys. Rev. D 63 (2001) 014022.

[48] A.I. Davydychev and V.A. Smirnov, Nucl. Instr. Meth. A502 (2003) 621. 
[49] L.V. Avdeev, Comput. Phys. Commun. 98 (1996) 15.

[50] M. Steinhauser, Comput. Phys. Commun. 134 (2001) 335.

[51] D.J. Broadhurst, Z. Phys. C54 (1992) 599.

[52] A.V. Kotikov, J. High Energy Phys. 09 (1998) 001;

J.M. Chung and B.K. Chung, Phys. Rev. D59 (1999) 105014; J. Korean Phys. Soc. 40 (2002) 435 (hep-ph/0203143).

[53] S. Groote, J.G. Körner and A.A. Pivovarov, Phys. Rev. D60 (1999) 061701.

[54] H.R.P. Ferguson, D.H. Bailey and S. Arno, Math. Comput. 68 (1999) 351.

[55] F. Jegerlehner and O.V. Tarasov, Nucl. Phys. B549 (1999) 481.

[56] T.H. Chang, K.J. Gaemers and W.L. van Neerven, Nucl. Phys. B202 (1982) 407;

A. Djouadi and C. Verzegnassi, Phys. Lett. B195 (1987) 265;

A. Djouadi, Nuovo Cim. A100 (1988) 357;

B.A. Kniehl, J.H. Kühn and R.G. Stuart, Phys. Lett. B214 (1988) 621;

B.A. Kniehl, Nucl. Phys. B347 (1990) 86 ;

A. Djouadi and P. Gambino, Phys. Rev. D49 (1994) 3499; D53 (1996) 4111(E).

[57] M. Veltman, Diagrammatica, Cambridge University Press, 1994;

D. Bardin and G. Passarino, The Standard Model in the Making, Clarendon, Oxford, 1999.

[58] F. Jegerlehner, M.Yu. Kalmykov and O. Veretin, Nucl. Phys. B (Proc. Suppl.) 116 (2003) 382 (hep-ph/0212003); Nucl. Instr. Meth. A502 (2003) 618.

[59] J. Fleischer and F. Jegerlehner, Phys. Rev. D23 (1981) 2001.

[60] F. Jegerlehner, M.Yu. Kalmykov and O. Veretin, Nucl. Phys. B641 (2002) 285.

[61] K.G. Chetyrkin and M. Steinhauser, Nucl. Phys. B573 (2000) 617.

[62] F. Jegerlehner and M.Yu. Kalmykov, Nucl. Phys. B676 (2004) 365.

[63] J. Blümlein, hep-ph/0311046. 